\title{
Further Analysis of the Passive Dynamics of the Compass Biped Walker and Control of Chaos via Two Trajectory Tracking Approaches
}

\author{
Essia Added, ${ }^{1}$ Hassène Gritli $\mathbb{D}^{1},{ }^{1,2}$ and Safya Belghith ${ }^{1}$ \\ ${ }^{1}$ Laboratory of Robotics, Informatics and Complex Systems (RISC-Lab, LR16ES07), National Engineering School of Tunis, \\ University of Tunis El Manar, BP. 37, Le Belvédère 1002, Tunis, Tunisia \\ ${ }^{2}$ Higher Institute of Information and Communication Technologies, University of Carthage, Borj-Cedria 1164, Tunis, Tunisia \\ Correspondence should be addressed to Hassène Gritli; grhass@yahoo.fr
}

Received 24 January 2021; Revised 20 August 2021; Accepted 10 September 2021; Published 4 October 2021

Academic Editor: Leo Y. Zhang

Copyright (c) 2021 Essia Added et al. This is an open access article distributed under the Creative Commons Attribution License, which permits unrestricted use, distribution, and reproduction in any medium, provided the original work is properly cited.

This work consists in analyzing and controlling the walk of the compass-type bipedal walker in order to stabilize its passive dynamic gait. The dynamic walking of the compass-gait walker is modeled by an impulsive hybrid nonlinear system. This impulsive hybrid nature is considered very complex as it can generate unwanted phenomena such as chaos and bifurcations. We show first by means of bifurcation diagrams and by varying the slope angle of the walking surface and also the length of the lower leg segment that the passive dynamic walking exhibits successive period-doubling bifurcations leading to chaos. Furthermore, in order to control chaos and hence obtain one-periodic walking behavior, we propose two control approaches based on tracking a desired trajectory. The first method consists in tracking the one-periodic passive dynamic walking generated by the compass model itself. The second control method lies in following a planned trajectory using the 4th-order Spline function. An optimization method is also achieved to design the parameters of the desired trajectory. Some features of the period-1 passive gait are used in the design of such Spline trajectory. Finally, we show some simulation results revealing the efficiency of the two proposed control methods in the control of the chaotic passive gait of the compass-gait walker. Moreover, we demonstrate the stabilization of the bipedal locomotion of the compass biped walker on different slopes: descending and ascending inclined planes and walking on a level ground. A comparison with the OGY-based control method is also performed to further show the superiority of these two control approaches.

\section{Introduction}

1.1. Background and Literature Review. Research in bipedal robotics has continued to evolve increasingly in these recent years. Many prototypes of biped robots were built worldwide, and then tasks performed by different researchers in the robotic community have become more complex and hence a challenging issue [1-3]. Concerning bipedal robotic walking, the latest studies have focused on the control of two-legged bipedal robots and also on the minimization of energy consumption. One goal that emerges from all these studies is to try to reproduce as much as possible the human walking. The major challenge is to construct a biped robot capable of walking on different types of ground. The researchers therefore tried to reproduce as closely as possible the human and his locomotion by means of a biped robot via control.

In the literature, there are several studies and methods for the design of effective control laws to stabilize the walking of a biped robot. The common point between these studies is the tracking of some desired reference trajectory, which should be designed. The design of reference trajectories for the walking cycles of the biped robot is important and not trivial. Several techniques have been adapted to define the reference trajectories. The mathematical basis for the generation of trajectories is mainly the polynomial calculus, which constructs the equation of motion from spatial and temporal constraints. Most of the biped robot 
control approaches proposed in the literature are based on the use of reference trajectories that must be followed in real time. This clearly shows the importance of a trajectory generator for bipedal walking. Among these control methods, we quote the following: methods based on simplified models which consist in using the $3 \mathrm{D}$ inverse pendulum model with one or more masses [4-7], methods derived from biomechanics [8,9], methods based on oscillators $[10,11]$, and methods based on splines [12]. Some other control approaches for biped robots can be found in [13].

As the control scheme of biped robots is generally applied during the swing phase of the bipedal walking, thus the biped robot seems like a double pendulum and then as a robot manipulator. Some other methods of trajectory planning for manipulator robots, which can be applied to biped robots, can be found, e.g., in [14-16]. Moreover, some other control methods for robotic manipulators can be found, for example, in $[17,18]$. An adaptive fuzzy full-state feedback control is proposed in [17] to enhance the tracking accuracy in a robotic manipulator with uncertainties. A reinforcement learning control strategy for flexible robot manipulators is developed in [18], and that is based on the actor-critic structure to enable vibration suppression while retaining trajectory tracking.

Chaos and bifurcations are complex phenomena that can appear in nonlinear dynamical systems and also in several fields [19-22]. It is known that these behaviors are exhibited in robotic systems, such as mechanical oscillators [23, 24], mobile robots [25], and biped robots [26]. For biped robots, the most found behavior is the route to chaos via a cascade of period-doubling bifurcations [26]. The first research group that reported such behavior in the passive dynamic walking of the compass-gait walker was that of Prof. Goswami and his coworkers $[27,28]$. After that, several works have shown the presence of such scenarios in other related biped models, see the review paper [26] and also the recent papers [29-37]. Moreover, it was shown in [38] that the passive dynamic walking of the compass robot exhibits the cyclic-fold bifurcation. The same bifurcation was revealed also in [39]. Furthermore, it was demonstrated in $[40,41]$ the exhibition of other attractive and complex behaviors and bifurcations in the dynamics of the passive compass biped under control, and it was demonstrated also in [42] the birth of the Neimark-Sacker bifurcation.

In the literature, there are several works achieved on the control of chaos in dynamic systems [43-47]. Some other recent control approaches can be found in [48-51]. However, a few works in the literature realized on control of chaos and bifurcations in the passive dynamics of biped robots, see, e.g., the two review papers $[26,52]$. The two main control methods are the Ott-Grebogi-Yorke (OGY) control and the delayed-feedback control (DFC). Some other control methods were also recently established in [53-57].

1.2. Motivations in Studying and Controlling the Passive Dynamic Walking. In research on biped robots, there are two kinds of walking; namely, static walking and dynamic walking, which is more difficult to realize than the first one. Moreover, passive dynamic walking has been shown to be an interesting approach to be employed as a reference or as a basis for the control of biped robots when the energy efficiency problem of the bipedal locomotion is posed [58-61]. McGeer showed in [62] that a simple two-link compass-type walker can walk down a slope without the need of motors, in contrast to other biped robots that require a lot of energy via motors to control every movement. He showed that its passive-dynamics walker can effectively mimic the human locomotion. After McGeer's works, many research studies have been achieved on the analysis and control of passive-dynamics biped robots $[27,33,52,58-61,63-76]$, just to mention a few. As reported in $[69,71]$, the passive dynamic walking of biped robots has mainly two motivations allowing and encouraging the researchers to study and use it. The first motivation is that the passive-dynamics biped robot presents a self-stabilized walking gait. The second motivation is that the use of the passive dynamic walking allows to increase the energy efficiency of the bipedal walking and then there is a considerable decrease of energy consumption for the controlled biped robot, which constitutes one of the main motivations of this work.

The passive compass-type bipedal robot is considered to be the simplest walking robot because its passive gait presents the first determinant of the human gait [27, 28]. However, addition of degrees of freedom increases the complexity of analytically solving the simulation models of the human body because the large number of actuators required to operate a biped robot increases considerably its weight. Thus, its simple morphology and efficiency make the compass-gait biped robot to be the quintessential model that can mimic human walking. Such compass biped robot model is without knees and ankles and with a point contact with the ground while walking. It is known that such bipedal walker is characterized by a passive dynamic walking, which is modeled by nonlinear impulsive hybrid dynamics $[27,28,52]$. This complex system of bipedal walking can reveal attractive and complex behaviors such as bifurcations and chaos [26-28].

Moreover, in order to mimic the human walk, it is necessary to make the walk of the compass-type bipedal robot periodic of period-1 for all values of different parameters. Therefore, it is necessary to apply a continuous control on the walk of the compass-type bipedal robot. The main objective behind the control of the bipedal walking is to mimic the human walking in order to develop efficient limbs requiring less energy for amputees. The limbs play an important role in rehabilitation. They are used by the disabled to their carriers to perform certain functionalities compensating for their deficiencies.

1.3. Objective, Novelty, and Contributions. In this work, we are mainly interested in the analysis and the control of the passive dynamic walking of the compass-gait walker. We first analyze the passive walk of the compass bipedal robot in order to understand the phenomena that may appear by 
varying certain parameters, which present in reality some kinds of disability that exist in the human body. Two parameters are adopted in this work, namely, the slope angle of the inclined walking surface and the length of the lower leg segment. For this last parameter, we study the case where the tight length and the shank length are not equal. To the best of our knowledge, such parameter was not considered in the literature to study the passive walking of the compass-type bipedal robot. We show via bifurcation diagrams that by varying these two parameters of the compass-gait walker, the period-doubling bifurcations and their subsequent route to chaos are revealed.

For the control of the compass-gait biped walker, we will propose two control approaches. The first control method lies in profiting of the benefits of the passive dynamic walking that are described previously, where among them there is the energy efficiency. Thus, the impulsive hybrid nonlinear model of the passive dynamic walking will be used in order to generate the desired period-1 gait/trajectory to follow by means of a controller. The second control approach consists in designing a desired period-1 gait/trajectory via a 4 th-order Spline polynomial function. In order to select the shortest path that can be traveled by the two legs of the compass biped walker, we added a constraint, and hence, the whole design problem of the 4 th-order Spline trajectory is transformed into a minimization of an objective function that represents the distance between the tip of the swing leg and the ground. Moreover, we will use two main descriptors of the period-1 passive gait of the compass walker, which are the state vector just before the impact with the walking surface and also the step period of such period-1 passive walking gait.

In order to track the desired trajectories planned/generated through the two proposed control approaches, we will consider the feedforward-plus-PD control law. Such controller is composed of the feedforward part, which is only used in the first control approach, and also the PD part implemented in the second control approach. Recently, in [77, 78], we applied, besides the feedforward-plus-PD controller, the computed torque control law and the gravity compensation-based PD control to stabilize the compassgait bipedal robot. Almost the same simulation results were obtained. The advantage behind choosing the feedforwardplus-PD control law is that it is simpler to realize in practice than the other two control laws.

We will show, via numerical simulations, that the first control approach based on the passive dynamic walking allows to control the chaotic gaits of the compass biped walker better with less energy consumption than the second control approach. Moreover, in order to show further the efficiency and superiority of the two proposed control approaches, we will realize a comparison with the OGY-based control method, which has been widely used in the control of chaos and the stabilization of the bipedal walking of the compass-gait walker. Such control method was recently developed in [72] using the Poincaré mapping approach, where authors developed an explicit mathematical expression of the controlled Poincaré map used mainly for the design of the control law.
The main contributions of this paper are summarized as follows:

(i) Study of the dynamic walking of the compass-type bipedal walker descending an inclined surface without control, that is to say with its natural passive gait, through bifurcation diagrams. The dynamics of such biped robot is governed by a complex model, namely, an impulsive hybrid nonlinear system.

(ii) Analysis of the walking behavior of the compass walker using, and for the first time, the length of the lower leg segment as a bifurcation parameter. We show hence the occurrence of the nonlinear phenomenon, the period-doubling route to chaos, and also the ability of the compass walker to go down steep slopes.

(iii) Stabilization of the chaotic passive walk of the compass-type bipedal robot via control into a period-1 gait, which is well selected and not any periodic trajectory. In fact, the desired period-1 gait selected for the control is embedded within the chaotic attractor and detected using the shooting method.

(iv) Proposition of two control strategies based on tracking a desired trajectory and also based on the properties of the desired period-1 passive bipedal walk. Indeed, for some sets of parameters of the biped robot, the desired period-1 passive gait and its associated walking descriptors are used in the control scheme.

(v) Use of the entire period-1 passive limit cycle generated by the impulsive hybrid nonlinear dynamics of the compass robot as the desired reference trajectory to be tracked in the first control approach.

(vi) Planning of a fourth-order Spline trajectory as the reference one in the second control approach and by using an optimization-based method. To plan such reference trajectory, we use the state vector just before impact, $x_{*}^{-}$, of the desired period-1 hybrid limit cycle and its associated step period, $\tau_{*}$, as two main descriptors of the passive gait.

(vii) Application of a feedforward-plus-PD control law to track the desired period- 1 reference trajectory.

(viii) Several simulation results and comparisons have been presented to show the efficiency and superiority of the two proposed control approaches in the stabilization of the passive gaits of the compass robot and then in the control chaos, compared with the OGY-based control method.

1.4. Structure of the Paper. The remainder of the paper is organized as follows. A description of the compass-gait walker is given in Section 2. In Section 3, an analysis of the passive gaits is realized. The two control approaches of the passive chaotic gaits of the compass walker are detailed in 
Section 4. The simulation results related to these control methods are presented in Section 5. In Section 6, a comparison with the OGY-based control method is performed. Finally, a conclusion and some future recommendations are provided, respectively, in Section 7 and 8.

\section{The Compass-Type Biped Walker and Its Dynamic Model}

2.1. Description of the Compass-Type Biped Walker. The compass-type biped walker is a two-link mechanical robotic system illustrated by Figure 1. It consists of two identical, rigid, straight legs that have neither knees nor ankles, and it is assumed that the two legs have a point contact with the ground. From a certain set of initial conditions, and while walking down the walking surface of slope $\varphi$, the biped walker does not use any source of actuation or control. It walks by virtue of gravity, and hence, it is completely passive [27, 28].

In Figure $1, m_{s}$ and $m_{n s}$ represent, respectively, the mass points of the support leg and the nonsupport leg. $m_{h}$ represents the mass point of the hip, which acts here as the upper body. The two legs are equal with $l=a+b$, where $a$ represents the distance from the tip of the leg to the centerof-mass of the leg, and $b$ is the distance from the hip to the center-of-mass. Moreover, $\theta_{s}$ represents the angle of the stance leg and $\theta_{n s}$ stands for the angular position of the swing leg. These angles are taken with respect to the vertical lines as indicated in Figure 1.

For simulating the passive dynamic walking of the compass-type biped walker, we will take in this work the inertial and geometrical parameters illustrated in Table 1.

2.2. Dynamic Model of the Compass Bipedal Walker. The bipedal walk of the compass-type walker is composed of two successive phases: (1) the swing phase (called also the simple support phase) and (2) the impact phase. The first phase begins with the support of the stance leg on the walking surface and ends when the swing leg strikes the ground and the support leg is hence lifted. However, the impact phase occurs when the nonsupport leg touches the ground with a nonzero angular velocity of the two legs. Such phase is due to the rigidity of the two legs and also the ground that dampens the impact and to the nonzero speed of contact between the swing leg and the walking surface. In the sequel, we present the equations that model the bipedal walking of the compass walker.

2.2.1. Dynamics Model of the Swing Phase. In this walking phase, the compass-gait walker is described as a doublependulum robotic system where the stance leg acts as a pivot with the ground. Thus, we use the Euler-Lagrange method to determine the dynamic model of the biped robot during this first phase. The Euler-Lagrange equation is given by the following expression:

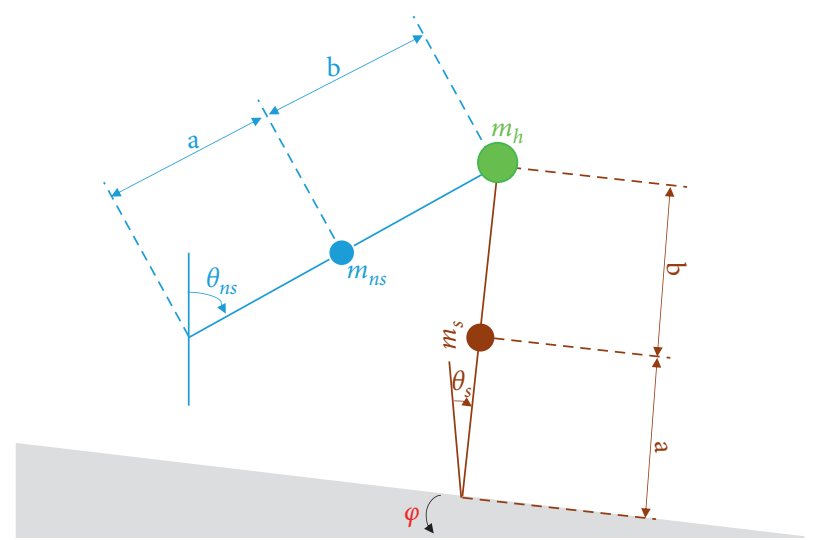

FIGURE 1: The passive compass-type biped walker on an inclined surface.

TABle 1: Parameters used for the simulation of the passive compass-type biped walker.

\begin{tabular}{lcc}
\hline Notation & Value & Unit \\
\hline$a$ & 0.5 & $\mathrm{~m}$ \\
$b$ & 0.5 & $\mathrm{~m}$ \\
$l$ & 1 & $\mathrm{~m}$ \\
$m$ & 5 & $\mathrm{Kg}$ \\
$m_{h}$ & 10 & $\mathrm{Kg}$ \\
$g$ & 9.8 & $\mathrm{~ms}^{-2}$ \\
\hline
\end{tabular}

$$
\frac{\mathrm{d}}{\mathrm{d} t}\left(\frac{\partial L(q, \dot{q})}{\partial \dot{q}}\right)-\frac{\partial L(q, \dot{q})}{\partial q}=\frac{\partial W}{\partial q},
$$

where $q=\left(\theta_{n s} \theta_{s}\right)^{T}, W$ stands for the work exerted via actuators on the biped robot while walking, and $L(q, \dot{q})=$ $E_{k}(q, \dot{q})-E_{p}(q)$ represents the Lagrangian function, with $E_{k}$ is the kinetic energy and $E_{p}$ is the potential energy.

Based on expression (1), we obtain the following nonlinear dynamics:

$$
J(q) \ddot{q}+C(q, \dot{q}) \dot{q}+G(q)=B u,
$$

with $J(q)$ is the inertia matrix:

$$
J(q)=\left[\begin{array}{cc}
m b^{2} & -m l b \cos \left(\theta_{s}-\theta_{n s}\right) \\
-m l b \cos \left(\theta_{s}-\theta_{n s}\right) & \left(m+m_{h}\right) l^{2}+m a^{2}
\end{array}\right] .
$$

$C(q, \dot{q})$ is the matrix of Coriolis and centrifugal forces:

$C(q, \dot{q})=\left[\begin{array}{cc}0 & m b l \sin \left(\theta_{s}-\theta_{n s}\right) \dot{\theta}_{s} \\ -m b l \sin \left(\theta_{s}-\theta_{n s}\right) \dot{\theta}_{n s} & 0\end{array}\right]$.

$G(q)$ is the vector of gravitational torques:

$$
G(q)=\left[\begin{array}{c}
-g m b \sin \left(\theta_{n s}\right) \\
g\left[\left(m+m_{h}\right) l+m a\right] \sin \left(\theta_{s}\right)
\end{array}\right] .
$$

$B$ is the control matrix: 


$$
B=\left[\begin{array}{cc}
-1 & 0 \\
1 & 1
\end{array}\right]
$$

and $u=\left[\begin{array}{l}u_{h} \\ u_{s}\end{array}\right]$ is the vector of control inputs, which will be designed next and used in order to control the passive dynamic walking of the compass-gait biped walker. Here, $u_{h}$ is the control input applied on the hip, and $u_{s}$ is the control input applied to the stance leg.

It is worth to note that for the passive dynamic walking of the compass biped robot, the control vector is zero, that is, $u=0$, and hence, the biped robot is not controlled, and the swing phase is therefore entirely passive.

2.2.2. Impact Phase. The dynamics of the impact phase is determined through the angular momentum conservation method $[27,28]$. For this, we consider the two following signs: "+" or "-" that will be placed to the right of a variable in order to refer, respectively, to the value of such variable just after and just before the impact of the swing leg of the biped robot with the ground.

The impact phase occurs when the previous swing phase is terminated, and this happens when the swing leg of the compass walker touches the walking surface. Relying first on Figure 1, it is straightforward to show that the impact event occurs when the following condition (7) on the angular positions of the two legs and the slope angle is well satisfied:

$$
\theta_{n s}+\theta_{s}+\varphi=0 .
$$

At the impact of the swing leg with the walking surface, there is a conservation of the angular momentum according to the following relation $[27,28]$ :

$$
Q_{p}(q) \dot{q}^{+}=Q_{m}(q) \dot{q}^{-},
$$

with $\dot{q}^{+}$and $\dot{q}^{-}$are the angular velocities just after and just before the impact, respectively, where

$$
\begin{aligned}
Q_{p}(q) & =\left[\begin{array}{c}
m\left(b^{2}-l b \cos \left(\theta_{s}-\theta_{n s}\right)\right) \\
m a^{2}+m_{h} l^{2}+m\left(l^{2}-l b \cos \left(\theta_{s}-\theta_{n s}\right)\right) \\
m b^{2}
\end{array}\right], \\
Q_{m}(q) & =\left[\begin{array}{cc}
-m a b & m_{h} l^{2} \cos \left(\theta_{s}-\theta_{n s}\right)+m\left(2 l a \cos \left(\theta_{s}-\theta_{n s}\right)-a b\right) \\
0 & -m a b
\end{array}\right] .
\end{aligned}
$$

\subsection{Impulsive Hybrid Nonlinear Dynamics of the Compass-} Gait Walker. We pose $x=\left[\begin{array}{ll}q & \dot{q}\end{array}\right]^{T}$ as the state vector. According to expressions (2), (7), and (8), the complete dynamics of the compass-type walker is defined by the following impulsive hybrid nonlinear dynamics:

$$
\begin{array}{ll}
\dot{x}=f(x)+g(x) \mathcal{u}, & \text { if } x \notin \Gamma, \\
x^{+}=h\left(x^{-}\right), & \text {if } x \in \Gamma,
\end{array}
$$

with $\quad f(x)=\left[\begin{array}{c}\dot{q} \\ -J(q)^{-1}(C(q, \dot{q}) \dot{q}+G(q))\end{array}\right], \quad g(x)=$ $\left[\begin{array}{c}0 \\ J(q)^{-1} B\end{array}\right]$, and $h(x)=\left[\begin{array}{c}R q \\ S(q) \dot{q}\end{array}\right]$, where the two matrices $R$ and $S$ are defined, respectively, as $R=\left[\begin{array}{ll}0 & 1 \\ 1 & 0\end{array}\right]$ and $S(q)=Q_{p}(q)^{-1} Q_{m}(q)$. Moreover, $\Gamma$ in the system (10a) and (10b) defines the impact set and is expressed like so

$$
\Gamma=\left\{x \in \mathbb{R}^{4}: C x+2 \varphi=0\right\},
$$

where $C=\left[\begin{array}{llll}1 & 1 & 0 & 0\end{array}\right]$.

\section{Analysis of the Passive Walking Dynamics of the Compass Biped Walker}

The impulsive hybrid nonlinear system describing the walking dynamics of the compass-gait biped walker is considered to be very complex to handle in analysis and that can generate complex attractive behaviors. The most common behavior revealed in the passive dynamic walking of the compass-gait walker as walking down the walking surface is the cascade of successive period-doubling bifurcations and their subsequent route to chaos. In this section, we will illustrate such behavior via bifurcation diagrams by varying two parameters, namely, the length $a$ of the lowerhalf segment of the legs and the slope $\varphi$ of the inclined surface. It is important to note that to the best of authors' knowledge, such parameter $a$ was not considered in previous works on the compass walker. Only some results are brief given in this paper. An in-depth study of the dynamics of the bipedal walking under variation of this bifurcation parameter $a$ will be developed in another work.

Next and before the analysis of the passive gaits of the compass biped walker, we present some brief details on the method used for determining the fixed point of the hybrid limit cycle for a given set of the two parameters $a$ and $\varphi$. In this work, we are only interested in determining the period-1 fixed points of the limit cycles and then of the passive gait.

We note first that for the passive gait of the compass walker, the control input $u$ in (10a) is zero. Thus, the continuous dynamics of the swing phase becomes

$$
\dot{x}=f(x), \quad \text { if } x \notin \Gamma \text {. }
$$

Moreover, we note

$$
x(t)=\phi\left(t, x_{0}\right),
$$

as the solution of the differential equation $\dot{x}=f(x)$ in (12) emanated from the initial condition $x_{0}$. 
3.1. Determination of the Period-1 Fixed Point of the Passive Walk and Its Stability. It is important to note that the period-1 fixed point defines the initial condition of the passive gait. The appropriate choice of such initial condition is to take it as the state vector just after the impact phase. Thus, to find the period-1 fixed point, we fix the Poincaré section to be the set $\Gamma$ defined in (11). The development presented in this section can be found in $[13,79]$ with necessary details.

The intersection of the continuous trajectory of the swing phase with the Poincare section $\Gamma$ defines a sequence of points just after the impact phase: $x_{1}^{+}, x_{2}^{+}, \ldots, x_{k}^{+}$, for $k \geq 1$. These points define implicitly the following expression of the Poincaré map [36]:

$$
x_{k+1}^{+}=P\left(x_{k}^{+}\right) \text {. }
$$

Our objective is to find the period-1 fixed point, noted $x_{*}^{+}$, of the passive gait of the compass-type biped walker. Thus, according to the Poincaré map (14), such fixed point should satisfy the following relation:

$$
x_{*}^{+}=P\left(x_{*}^{+}\right) .
$$

To find this period-1 fixed point $x_{*}^{+}$, we must solve the following equation:

$$
H\left(x_{*}^{+}\right)=P\left(x_{*}^{+}\right)-x_{*}^{+}=0
$$

To find the period-1 fixed point $x_{*}^{+}$, we use the Newton-Raphson algorithm to solve this previous nonlinear equation (16) and then the following iterative scheme:

$$
x_{i+1}^{+}=x_{i}^{+}-D H^{-1}\left(x_{i}^{+}\right) \times H\left(x_{i}^{+}\right),
$$

where $D H\left(x^{+}\right)$is the Jacobian matrix of the function $H\left(x^{+}\right)$, and it is defined by the following expression:

$$
D H\left(x^{+}\right)=\left(\mathscr{I}_{n}-\frac{f\left(x\left(\tau_{r}\left(x^{+}\right)\right)\right) C}{C f\left(x\left(\tau_{r}\left(x^{+}\right)\right)\right)}\right) \Phi^{+}\left(\tau_{r}\left(x^{+}\right), x^{+}\right)-\mathscr{I}_{n},
$$

where $\tau_{r}\left(x^{+}\right)$stands for the return time of the trajectory of the swing phase emanated from the state $x^{+}$, to the Poincaré section $\Gamma$. Thus, the quantity $\tau_{r}\left(x^{+}\right)$defines the time from the state $x_{i}^{+}$to the new state $x_{i+1}^{+}$. As the impact phase is instantaneous, then $\tau_{r}\left(x^{+}\right)$is the time from $x_{i}^{+}$to $x_{i+1}^{-}$. In (18), $\mathscr{I}_{n}$ is the identity matrix.

Moreover, $\Phi^{+}\left(\tau_{r}\left(x^{+}\right), x^{+}\right)$denotes the monodromy matrix, which is the fundamental solution matrix $\Phi\left(t, x^{+}\right)$ evaluated just after the impact phase. Because of the impact phase, such matrix $\Phi\left(t, x^{+}\right)$undergoes a jump and it is then related to the fundamental solution matrix just before the impact, noted $\Phi^{-}\left(\tau_{r}\left(x^{+}\right), x^{+}\right)$, via the jump matrix noted $S$ as follows:

$$
\Phi^{+}\left(\tau_{r}\left(x^{+}\right), x^{+}\right)=S\left(x^{+}, x^{-}\right) \Phi^{-}\left(\tau_{r}\left(x^{+}\right), x^{+}\right),
$$

where $x^{-}$denotes the state just before the impact.

The matrix $\Phi^{-}\left(\tau_{r}\left(x^{+}\right), x^{+}\right)$in (19) is the fundamental solution matrix evaluated just before the impact. Such matrix is defined and computed according to the following expression:

$$
\Phi^{-}\left(\tau_{r}\left(x^{+}\right), x^{+}\right)=\frac{\partial \phi\left(\tau_{r}\left(x^{+}\right), x^{+}\right)}{\partial x^{+}},
$$

where $\phi\left(\tau_{r}\left(x^{+}\right), x^{+}\right)$is the solution during the swing phase and is defined previously by expression (13).

The jump matrix $S\left(x^{+}, x^{-}\right)$in (19) is defined by the following expression:

$$
S\left(x^{+}, x^{-}\right)=h_{x}^{-}-\frac{\left(h_{x}^{-} f^{-}-f^{+}\right) C}{C f^{-}},
$$

where $h_{x}^{-}=(\partial h / \partial x)\left(x^{-}\right)$, with $h(x)$ is the nonlinear function given in the algebraic equation of the impact phase in (10b).

By choosing an initial guess $x_{0}^{+}$for iterative scheme (17), this last will converge then after some iterations to the period-1 fixed point $x_{*}^{+}$. For such fixed point $x_{*}^{+}$, it corresponds the fixed step period $\tau_{*}$, which is $\tau_{r}\left(x_{*}^{+}\right)$.

The stability of this fixed point $x_{*}^{+}$is investigated via the eigenvalues of the Jacobian matrix of the Poincaré map. Relying on expression (16) and (18), the Jacobian matrix of the Poincaré map, noted $D P\left(x^{+}\right)$, is defined by the following expression:

$$
D P\left(x^{+}\right)=\left(\mathscr{I}_{n}-\frac{f\left(x\left(\tau_{r}\left(x^{+}\right)\right)\right) C}{C f\left(x\left(\tau_{r}\left(x^{+}\right)\right)\right)}\right) \Phi^{+}\left(\tau_{r}\left(x^{+}\right), x^{+}\right) .
$$

If all the eigenvalues of the Jacobian matrix $D P\left(x^{+}\right)$ evaluated at the period-1 fixed point $x_{*}^{+}$are strictly inside the unit circle, then such fixed point $x_{*}^{+}$is stable. However, if at least only one eigenvalue is outside the unit circle, then the period-1 fixed point is unstable.

3.2. Behavior of the Passive Gait for $a=0.5$ and as $\varphi$ Varies. In this first analysis, we fix the parameter $a$ to $a=0.5$, which is the classical value adopted in the literature for the two legs of the compass biped robot. Thus, by varying the slope $\varphi$, we obtain the bifurcation diagram in Figure 2, which reveals the period-doubling route to chaos. Initially and for small angles of the slope, the passive gait is with period-1. An increase of the slope $\varphi$ induces the appearance of the first perioddoubling bifurcation at the slope $\varphi=4.391^{\circ}$. A further increase of $\varphi$ causes then the exhibition of subsequent perioddoubling bifurcations, which lead to the formation of chaos. At the value of the slope $\varphi=5.21^{\circ}$, the chaotic behavior is terminated provoking hence the fall of the biped robot. Notice that at the first period-doubling bifurcation, the period-1 passive gait, which is initially stable, becomes unstable. Such period-1 unstable passive gait is depicted in Figure 2 with the dashed curve. Notice that after the critical value $\varphi=5.21^{\circ}$, only the unstable motions with different period numbers exist, and then the period-1 unstable passive gait continues to exist.

It is worth to note that here in Figure 2 and also in Figures 3 and 4 presented in the two next sections, the dashed curves reveal the unstable period-1 fixed point of the bipedal gait. Such fixed point was determined according to the previous section. 


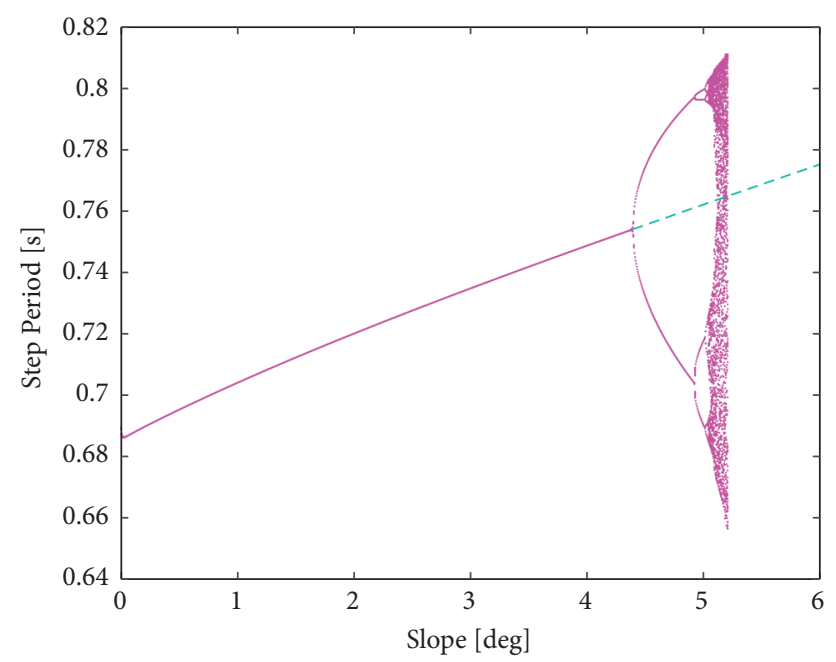

FIGURE 2: Bifurcation diagram showing the step period of the passive gait as the slope parameter $\varphi$ varies and for the fixed parameter $a=0.5$.

In Figure 5, we plotted two different behaviors in the state space. Figure 5(a) reveals a one-periodic attractor, namely, the period-1 passive hybrid limit cycle obtained for the slope $\varphi=3^{\circ}$, whereas Figure 5(b) shows a chaotic attractor depicted for the slope $\varphi=5.2^{\circ}$. In these figures, the pink curve reveals the behavior of the swing leg, and the cyan curve represents the motion of the support leg. However, the green lines show the transition event that occurs at the impact phase.

3.3. Behavior of the Passive Gait for $\varphi=4^{\circ}$ and as a Varies. In this part, we fix the slope angle at the value $\varphi=4^{\circ}$ and we vary the parameter $a$. As a result, we obtain the bifurcation diagram in Figure 6. Note that, according to the bifurcation diagram in Figure 2, for $\varphi={ }^{4^{\circ}}$ and $a=0.5$, the passive gait is of period-1. Then, by increasing and decreasing the value of $a$ from this nominal value $a=0.5$, we obtained the scenario in Figure 6. It is obvious that by increasing $a$, only the period-1 passive gaits have been generated. These period-1 gaits were found to be terminated at the value $a=0.9936$ and then leading to the fall of the compass biped. However, by decreasing the value of the parameter $a$, we observe the exhibition of the period-doubling route to chaos. The first period-doubling bifurcation occurs at the value $a=0.4615$, at which the period-1 stable gait becomes unstable. The chaotic motions were found to be ended at the value $a=$ 0.3995 and hence causing the fall of the biped robot.

Relying on the bifurcation diagram in Figure 6, we selected two values of the parameter $a$ in order to show the resultant behavior in the state space. Figure 4(a) shows the period-1 passive limit cycle depicted for $a=0.8$, whereas Figure 4(b) reveals the chaotic attractor plotted for the parameter $a=0.4$. It is obvious that the period- 1 limit cycle in Figure 4(a) is larger than that in Figure 5(a). Moreover, at the impact phase, the transition in the velocity of the two legs in Figure 4(a) is more important (bigger) than that in Figure 5(a). This is because there is a considerable difference between the two values of the parameter $a: a=0.5$ in Figure 5(a) and $a=0.8$ in Figure 4(a). However, there is no clear difference between the chaotic attractor in Figure 5(b) and that in Figure 4(b). Almost the same form was obtained.

3.4. Behavior of the Passive Gait for $a=0.8$ and as $\varphi$ Varies. In this analysis, we fix the parameter $a$ to the value $a=0.8$. As reported in Figure 6, for such value of $a$, the behavior of the passive walking of the compass biped robot is periodic of period-1. Then, emanating from this value of $a$, we varied the parameter $\varphi$. As a result, we obtained the bifurcation diagram in Figure 3. Obviously, the passive walking dynamics of the biped robot experiences the period-doubling route to chaos as $\varphi$ increases. This behavior is emanated from the period-1 gait at the near-zero slope and continues to the chaotic motion, which is terminated at the slope $\varphi=8.337^{\circ}$.

In Figure 7(a), we plotted the period-1 limit cycle obtained for the slope angle $\varphi=6^{\circ}$, whereas in Figure 7(b), we present the chaotic attractor obtained for the slope $\varphi=8.3^{\circ}$. It is widely clear the difference between the chaotic attractor in Figure 7(b) and that in Figure 4(b). In Figure 4(b), the maximum angular position that can be reached by the swing leg is around $26^{\circ}$. However, the maximum angular position for the present case in Figure $7(\mathrm{~b})$ is around $70^{\circ}$. Moreover, in Figure 4(b), the impact of the swing leg occurs with an angular position almost equal to $12^{\circ}$, whereas in Figure 7(b), the angular position of the swing leg at the impact is about ${ }^{5^{\circ}}$. This shows that the step length of the biped robot, in this case of study, becomes smaller than that investigated in the previous case, which is given in Figure 4(b).

\section{Control Approaches of the Compass-Gait Biped Walker}

As discussed previously, the passive dynamic walking of the compass-gait biped walker exhibits a period-doubling route to chaos, and hence, unstable period-1 passive gaits are generated through the first period-doubling bifurcation. Note that the period- $p$ gaits, for $p>1$, are all asymmetric, and then only the period-1 gaits are symmetric and that resemble to that of the human walking. In order to eliminate 


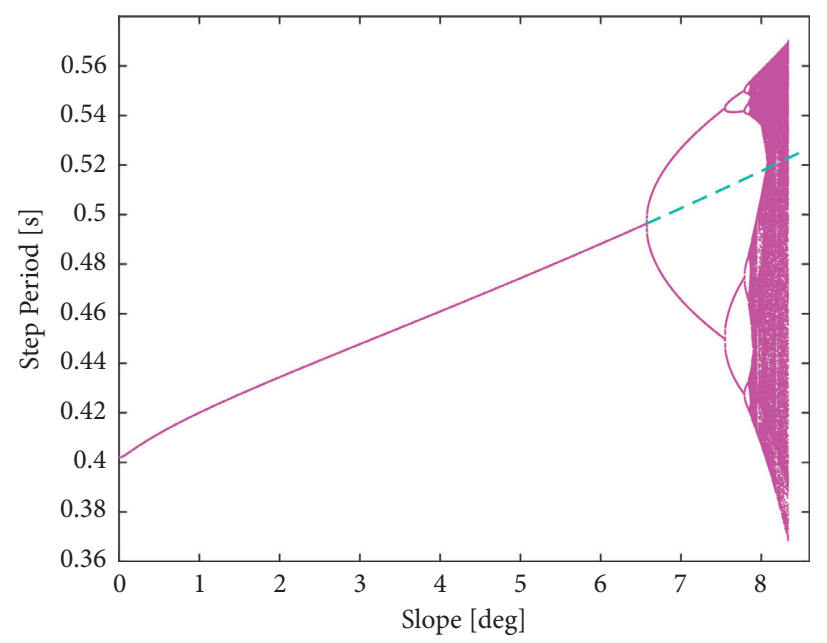

(a)

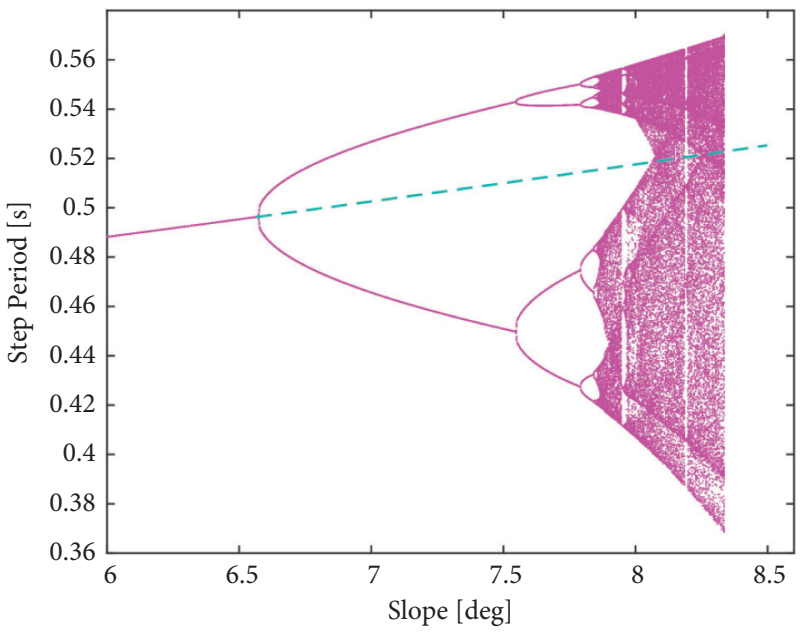

(b)

FiguRE 3: Bifurcation diagram showing the behavior of the passive gaits of the compass-gait walker for the fixed parameter $a=0.8$ and as $\varphi$ varies. (b) An enlargement of (a) demonstrating the period-doubling route chaos.

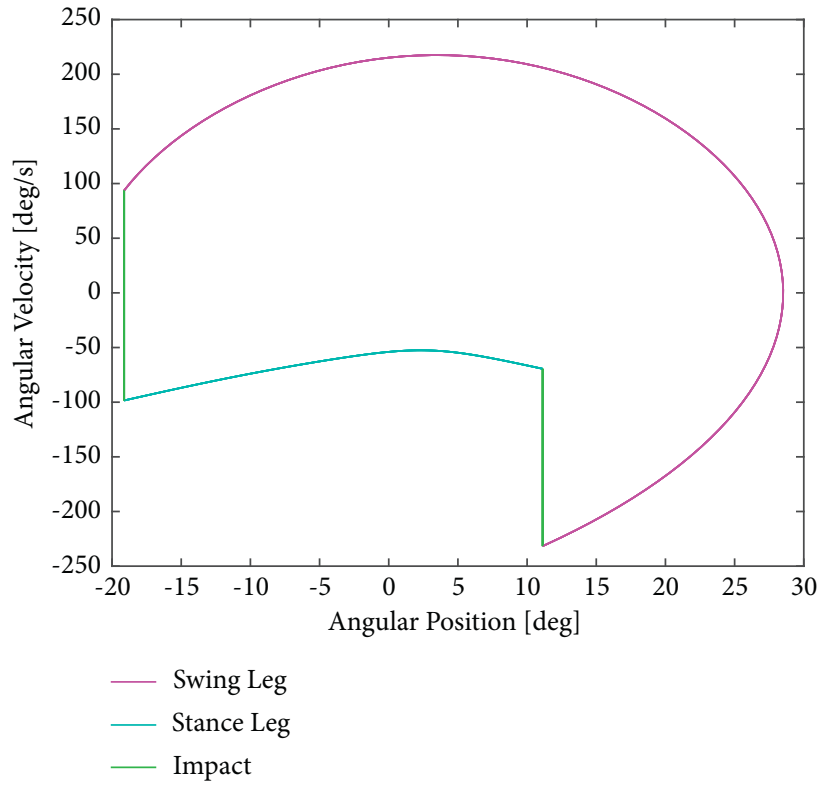

(a)

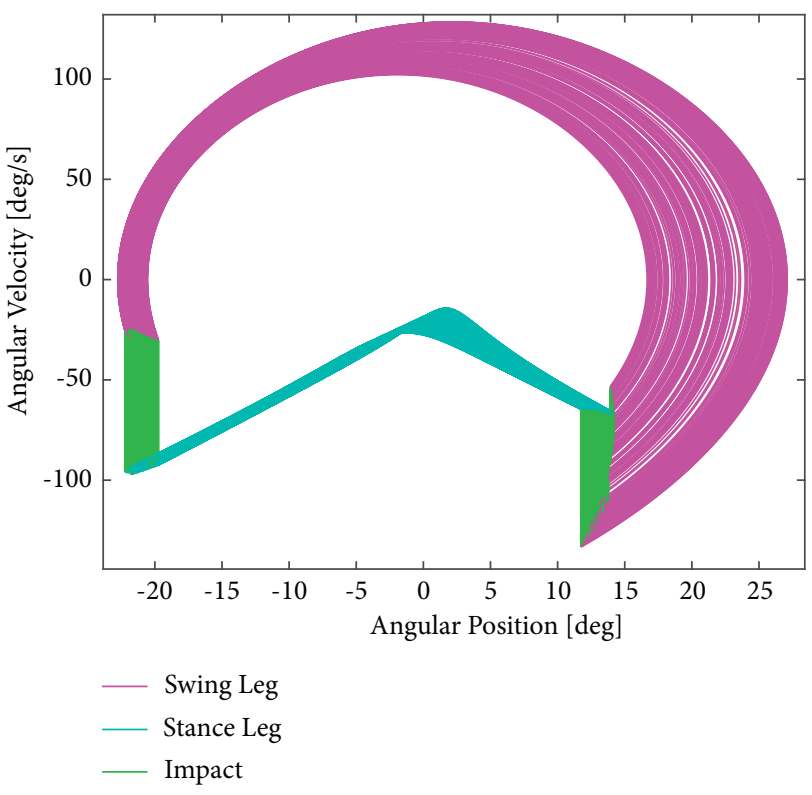

(b)

FIgURE 4: Attractors plotted for the fixed slope $\varphi=4^{\circ}$ and for two different values of the parameter $a$. (a) $a=0.8$. (b) $a=0.4$.

the chaotic behavior and also in order to control these asymmetric gaits, we need to apply a well-designed control law $u$ to introduce in the impulsive hybrid nonlinear dynamics (10a) and (10b).

4.1. Methodology and Adopted Control Law. In order to control the passive gaits of the compass-gait biped robot into a period-1 gait for all values of the lower leg segment length $a$ and of the slope angle of the walking surface $\varphi$, we will apply two approaches. The first control approach is mainly based on the passive dynamic walking. Indeed, we will use the period-1 passive gait that can be either stable or unstable, as a reference trajectory to be tracked by the biped robot during the swing phase. The second control approach lies in the design of a trajectory by our-self using the Spline polynomial method. Such planned trajectory will be also used as a desired one to be tracked by the biped robot. These two control approaches will be detailed in the sequel.

To define the reference trajectory, we need to generate/ design the desired joint variables $q_{d}(t), \dot{q}_{d}(t)$, and $\ddot{q}_{d}(t)$. Here, the term generate is used for the first control approach since these variables will be generated via the model of the passive dynamic walking. However, the term design is used for the second control approach since these variables will be obtained by means of a well-designed trajectory using the 


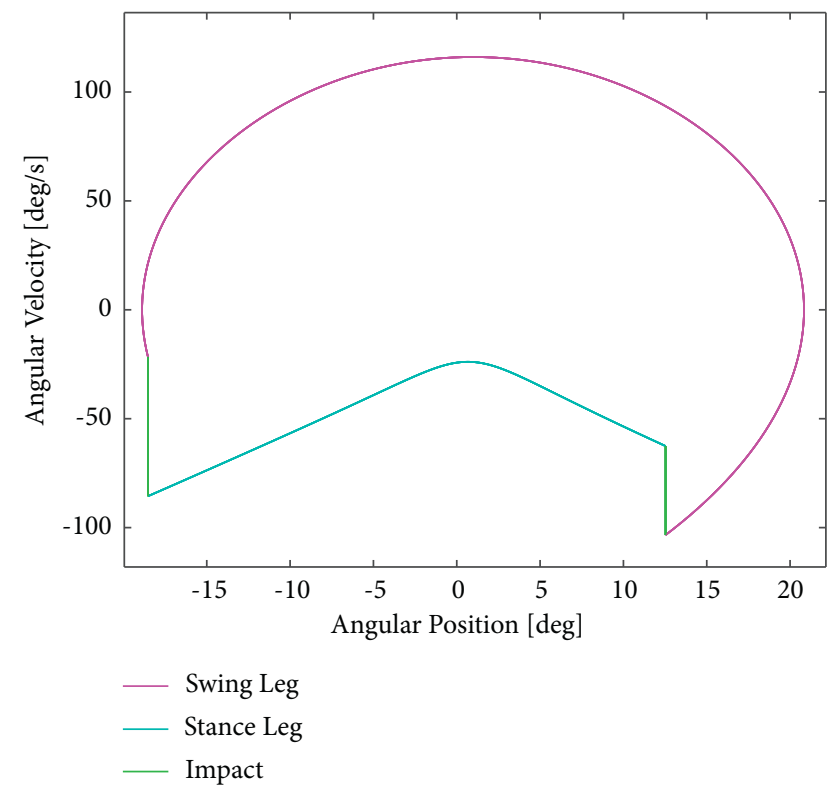

(a)

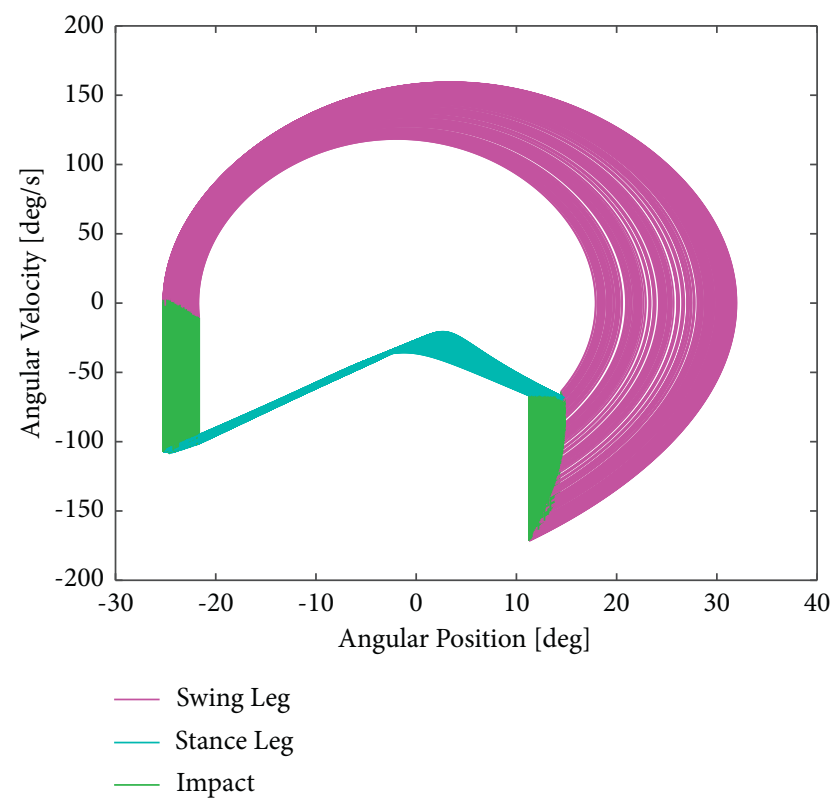

(b)

Figure 5: Attractors of the passive gait of the compass-gait walker for $a=0.5$ and for two different values of the slope $\varphi$. (a) $\varphi=3^{\circ}$. (b) $\varphi=5.2^{\circ}$.

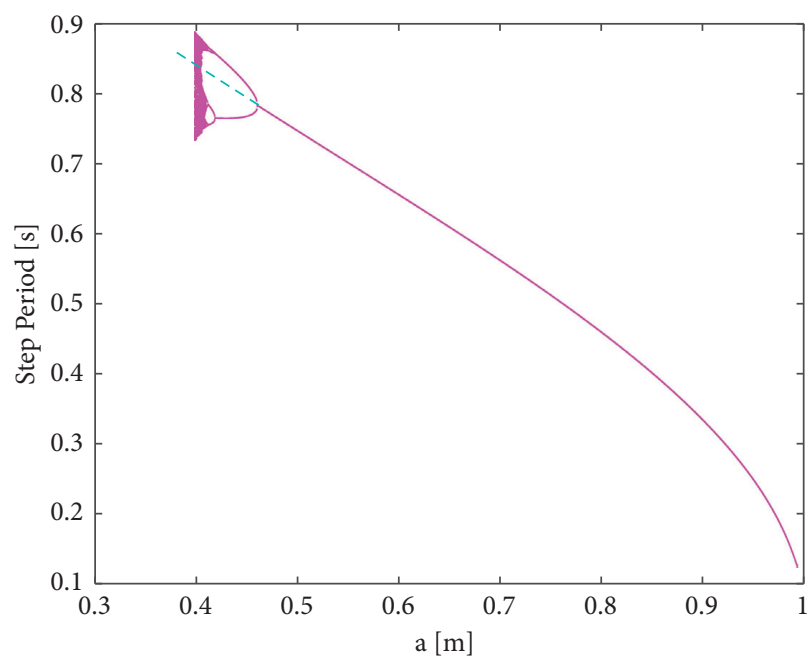

(a)

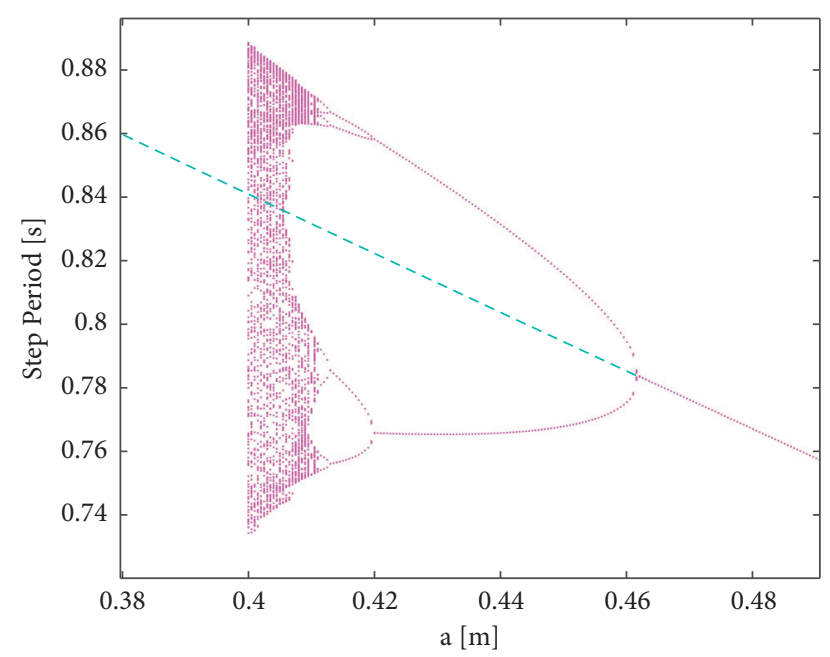

(b)

FIGURE 6: Bifurcation diagram revealing the behavior of the passive walking dynamics of the compass-gait biped walker as the parameter $a$ varies and for the fixed slope angle $\varphi=4^{\circ}$. (b) An enlargement of (a) showing clearly the period-doubling route to chaos.

Spline polynomial function. To control the gait of the compass walker to follow the desired period-1 trajectory defined mainly by the desired joint variable $q_{d}(t)$, we will use a compound tracking control law, which is called as the feedforward-plus-PD (FF + PD) controller, and which is defined as follows:

$$
u(t)=u_{\mathrm{FF}}+u_{\mathrm{PD}}
$$

where $u_{\mathrm{FF}}$ is the feedforward control law and is defined by the following expression:

$$
u_{\mathrm{FF}}=B^{-1}\left(J\left(q_{d}\right) \ddot{q}_{d}+C\left(q_{d}, \dot{q}_{d}\right) \dot{q}_{d}+G\left(q_{d}\right)\right),
$$

and $u_{\mathrm{PD}}$ is the proportional-derivative (PD) control law defined as follows:

$$
u_{\mathrm{PD}}=-B^{-1}\left(K_{p} e(t)+K_{v} \dot{e}(t)\right)
$$

where $e(t)$ and $\dot{e}(t)$ are, respectively, the tracking error and its derivative, with $e(t)=q(t)-q_{d}(t)$ and $\dot{e}(t)=\dot{q}(t)-$ $\dot{q}_{d}(t)$. Moreover, $K_{p}$ and $K_{v}$ are two symmetric positivedefinite matrices. 


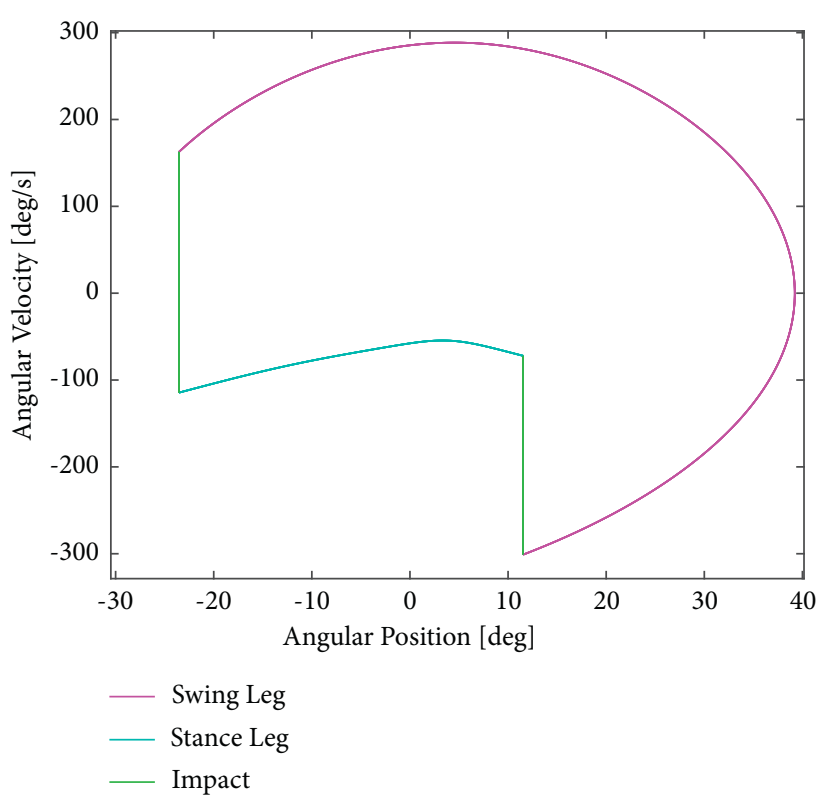

(a)

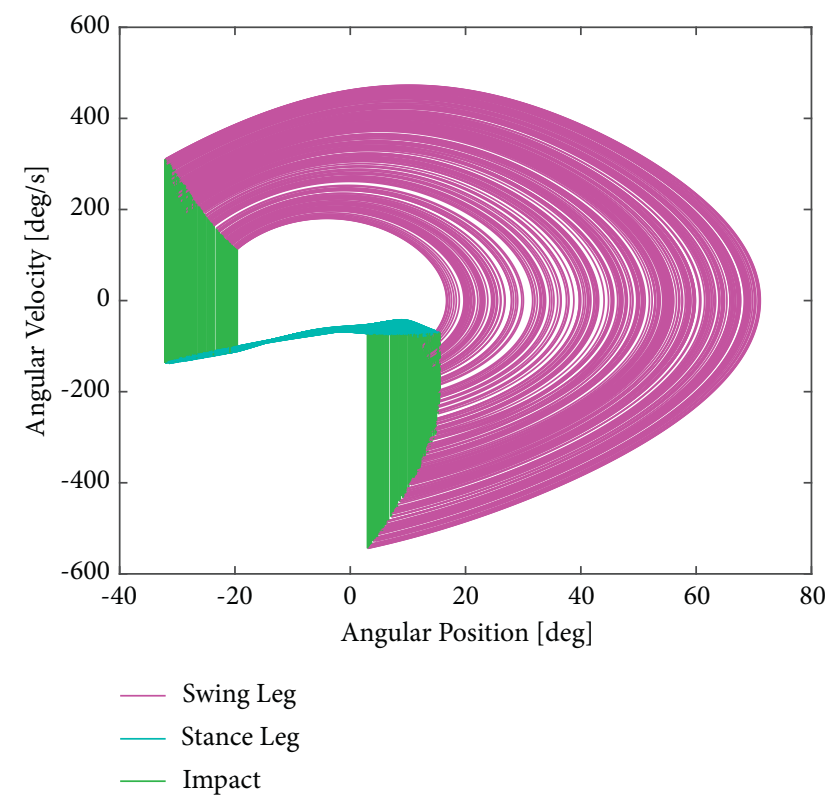

(b)

Figure 7: Two different attractors obtained for two different slopes and for the parameter $a=0.8$. (a) A period-1 hybrid limit cycle plotted for $\varphi=6^{\circ}$, whereas (b) a chaotic attractor depicted for $\varphi=8.3^{\circ}$.

It is worth to mention that the subcontroller $u_{\mathrm{FF}}$ in (24) or the subcontroller $u_{\mathrm{PD}}$ in (25) can be applied solely. Thus, we will have two possible control laws that can be used to control the passive gaits of the compass biped robot. However, the FF + PD compound controller in (23) will be, in general, the improved one.

Moreover, for adopted control law (23), the symmetric positive-definite matrices $K_{p}$ and $K_{v}$ are taken as

$$
\begin{aligned}
& K_{p}=\operatorname{diag}\left\{w_{1}^{2}, w_{2}^{2}\right\}, \\
& K_{v}=\operatorname{diag}\left\{2 w_{1}, 2 w_{2}\right\},
\end{aligned}
$$

where the values of $w_{1}$ and $w_{2}$ will be selected arbitrary.

Remark 1. It is worth to note that the design parameters $K_{p}$ and $K_{v}$ of the adopted control law, namely, the FF+ PD controller (23), are selected according to expressions (26) and (27). The main objective behind the choice of these expressions of $K_{p}$ and $K_{v}$ is to obtain in the closed loop a second-order polynomial function with a damping ratio, saying $\zeta$, equal to 1 . This choice of $\zeta$ is in fact in order to avoid some little overshoot and hence to obtain a critical damping with a rapid response of the controlled biped robot during the swing phase.

4.2. First Approach: Control Based on the Passive Dynamic Walking. In this first control approach based on the passive dynamic walking of the compass biped walker, the desired joint variables $q_{d}(t), \dot{q}_{d}(t)$, and $\ddot{q}_{d}(t)$, which define the period-1 passive trajectory and which will be used in FF + PD control law (23), are generated from the dynamic model of the passive walking during the swing phase. Thus, according to Section 2 and from dynamics (2) and for a passive gait, that is, for $u=0$, the desired dynamics that generates the desired period-1 passive trajectory along the swing phase is defined as follows:

$$
J\left(q_{d}\right) \ddot{q}_{d}+C\left(q_{d}, \dot{q}_{d}\right) \dot{q}_{d}+G\left(q_{d}\right)=0 .
$$

Moreover, in order that such system (28) generates the period-1 passive trajectory, we should well choose the initial condition just after the impact phase, $x_{0}^{+}$. Actually, in order to have exactly the period-1 passive gaits that are investigated in the previous section, the trajectory should start with the period-1 fixed point $x_{*}^{+}$, which was already determined according to Section 3.1. Moreover, we emphasize that as the period-one fixed point $x_{*}^{+}$can be unstable for some values of the biped's parameters, then and in order to ensure the desired period-1 passive gait to be same at each new step, dynamic model (28) should always start with the same initial condition, that is with the same period-1 fixed point $x_{*}^{+}$. Furthermore, such dynamics will end and then start again with the same initial condition $x_{*}^{+}$, if the swing leg of the compass-gait biped walker under the controller $u$, touches the walking surface. Hence, the complete model of the controlled dynamic walking of the compass robot using this first control approach is defined as follows: 


$$
\left\{\begin{array}{l}
\frac{\mathrm{d}}{\mathrm{d} t}\left[\begin{array}{l}
q \\
\dot{q}
\end{array}\right]=f(q, \dot{q})+g(q) u\left(q, \dot{q}, q_{d}, \dot{q}_{d}, \ddot{q}_{d}\right) \\
\ddot{q}_{d}=-J^{-1}\left(q_{d}\right)\left(C\left(q_{d}, \dot{q}_{d}\right) \dot{q}_{d}+G\left(q_{d}\right)\right)
\end{array}\right.
$$

$$
\left\{\begin{array}{l}
{\left[\begin{array}{l}
q^{+} \\
\dot{q}^{+}
\end{array}\right]=h\left(\left[\begin{array}{l}
q^{-} \\
\dot{q}^{-}
\end{array}\right]\right), \quad \text { if } x=\left[\begin{array}{l}
q \\
\dot{q}
\end{array}\right] \in \Gamma .} \\
{\left[\begin{array}{l}
q_{d}^{+} \\
\dot{q}_{d}^{+}
\end{array}\right]=x_{*}^{+}}
\end{array}\right.
$$

Note that in (29), $u\left(q, \dot{q}, q_{d}, \dot{q}_{d}, \ddot{q}_{d}\right)$ is the FF + PD controller $u(t)$ defined by expression (23). Moreover, we stress that in this first control approach based on the passive dynamic walking of the compass robot, and relying on the desired model defined by expression (28) to track, the feedforward controller $u_{\mathrm{FF}}$ in $(24)$ is zero $\left(u_{\mathrm{FF}}=0\right)$ and then has no action on the tracking problem of the period-1 passive gait. Hence, only PD controller (25) will be considered. As a result, we will have

$$
u(t)=u_{\mathrm{PD}}=-B^{-1}\left(K_{p} e(t)+K_{v} \dot{e}(t)\right) .
$$

\subsection{Second Approach: Control Based on Tracking a Designed Spline Trajectory}

4.3.1. Presentation of the Control Approach. The design of a reference trajectory for the walking cycles of the biped robot is important $[80,81]$. Our goal is to design a one-periodic trajectory that satisfies a certain objective in terms of position and velocity along the swing phase. As the compass biped walker has two links, we will then design a period-1 reference trajectory for each link, which is a reference trajectory for the swing leg and another one for the stance leg. In order to design such period-1 trajectories, we adopt the Spline polynomial method, for which the trajectory depends on the order (of time). In order to design these one-periodic reference trajectories, we mainly need the angular position and the angular velocity of each leg just after and just before the impact phase.

In the sequel, let $\theta_{i, 0}=\theta_{i}\left(t_{0}\right)$, for $i=\{n s, s\}$, be the initial joint position just after the impact phase for $t=t_{0}$. Let also $\theta_{i, f}=\theta_{i}\left(t_{f}\right)$ be the final joint position just before the impact phase for $t=t_{f}$. Moreover, at these times $t_{0}$ and $t_{f}$, it corresponds the joint velocity $\theta_{i, 0}=\dot{\theta}_{i}\left(t_{0}\right)$ and $\theta_{i, f}=\dot{\theta}_{i}\left(t_{f}\right)$.

It is worth to note that the time $t_{f}$ will be fixed as the desired step period of the passive dynamic walking of the compass biped robot. Thus, we will have $t_{f}=\tau_{*}$. Moreover, the final angular positions and velocities of the two legs, that is, $\theta_{i, f}$ and $\theta_{i, f}$ with $i=\{n s, s\}$, will be chosen to be the state of the period-1 fixed point just before the impact phase. Such fixed point is noted as $x_{*}^{-}$. Thus, in the sequel, we will use the following notions: $\theta_{i, *}^{-}=\theta_{i, f}$ and $\dot{\theta}_{i, *}^{-}=\theta_{i, f}$.

In fact, it is possible to choose other values for $t_{f}$, but as our objective is to control the passive gaits, then we will be interested in the features of the period-1 passive gaits, which are defined by their own period- 1 fixed point $x_{*}^{-}$and also their own step period $\tau_{*}$.
4.3.2. Design of the Order-4 Spline Reference Trajectory. Our goal in this section is to design an order-4 Spline trajectory to be tracked by the compass-gait walker. Such trajectory has the following form:

$$
\theta_{i}(t)=e_{i} t^{4}+d_{i} t^{3}+c_{i} t^{2}+b_{i} t+a_{i}
$$

where $a_{i}, b_{i}, c_{i}, d_{i}$, and $e_{i}$, are scalars to be designed next.

Then, by substituting all the initial and final conditions, we obtain

$$
\left\{\begin{array}{l}
\theta_{i, 0}=e_{i} t_{0}^{4}+d_{i} t_{0}^{3}+c_{i} t_{0}^{2}+b_{i} t_{0}+a_{i}, \\
\theta_{i, f}=e_{i} t_{f}^{4}+d_{i} t_{f}^{3}+c_{i} t_{f}^{2}+b_{i} t_{f}+a_{i}, \\
\dot{\theta}_{i, 0}=4 e_{i} t_{0}^{3}+3 d_{i} t_{0}^{2}+2 c_{i} t_{0}+b_{i}, \\
\dot{\theta}_{i, f}=4 e_{i} t_{f}^{3}+3 d_{i} t_{f}^{2}+2 c_{i} t_{f}+b_{i} .
\end{array}\right.
$$
follows:

As $t_{0}=0$ and $t_{f}=\tau_{*}$, the equations in (33) are recast as

$$
\left\{\begin{array}{l}
\theta_{i, 0}=a_{i} \\
\theta_{i, *}^{-}=e_{i} \tau_{*}^{4}+d_{i} \tau_{*}^{3}+c_{i} \tau_{*}^{2}+b_{i} \tau_{*}+a_{i} \\
\dot{\theta}_{i, 0}=b_{i} \\
\dot{\theta}_{i, *}^{-}=4 e_{i} \tau_{*}^{3}+3 d_{i} \tau_{*}^{2}+2 c_{i} \tau_{*}+b_{i}
\end{array}\right.
$$

These previous conditions in (34) can be rearranged in the following matrix form:

$$
\left[\begin{array}{l}
\theta_{i, 0} \\
\theta_{i, *}^{-} \\
\dot{\theta}_{i, 0} \\
\dot{\theta}_{i, *}^{-}
\end{array}\right]=\left[\begin{array}{ccccc}
1 & 0 & 0 & 0 & 0 \\
1 & \tau_{*} & \tau_{*}^{2} & \tau_{*}^{3} & \tau_{*}^{4} \\
0 & 1 & 0 & 0 & 0 \\
0 & 1 & 2 \tau_{*} & 3 \tau_{*}^{2} & 4 \tau_{*}^{3}
\end{array}\right]\left[\begin{array}{c}
a_{i} \\
b_{i} \\
c_{i} \\
d_{i} \\
e_{i}
\end{array}\right] .
$$

Notice that linear matrix equality (35) with unknown scalars $a_{i}, b_{i}, c_{i}, d_{i}$, and $e_{i}$ admits an infinity of solutions. Then, the desired trajectories to impose to be traveled by the two legs can have several possible paths. Therefore, the objective is to find the optimal trajectory allowing the tip of the swing leg of the compass biped robot to travel the shortest trajectory from the just-after-impact state to the just-before-impact state, which is from the state $x_{0}=\left[\begin{array}{llll}\theta_{n s, 0} & \theta_{s, 0} & \dot{\theta}_{n s, 0} & \dot{\theta}_{s, 0}\end{array}\right]^{T}$, which can be either just-afterimpact or any state at the swing phase, to the state just before impact $x_{*}^{-}=\left[\begin{array}{llll}\theta_{n s, *}^{-} & \theta_{s, *}^{-} & \dot{\theta}_{n s, *}^{-} & \dot{\theta}_{s, *}^{-}\end{array}\right]^{T}$.

Thus, in order of find the optimal values of these constants $a_{i}, b_{i}, c_{i}, d_{i}$, and $e_{i}$, for $i=\{n s, s\}$, we should minimize the following criterion:

$$
\mathscr{L}=\int_{0}^{\tau_{*}}\left(\theta_{n s}(t)+\theta_{s}(t)+2 \varphi\right)^{2} \mathrm{~d} t .
$$

From the conditions in (34), it follows that the two constants $a_{i}$ and $b_{i}$ are well defined as follows:

$$
\begin{aligned}
& a_{i}=\theta_{i, 0}, \\
& b_{i}=\dot{\theta}_{i, 0} .
\end{aligned}
$$


However, the three parameters $c_{i}, d_{i}$, and $e_{i}$ depend on each other. We adopt the constant $c_{i}$ to be the unknown parameter to be optimized according to the previous objective by minimizing the criterion $\mathscr{L}$ in (36). Thus, we need to define the two constants $d_{i}$ and $e_{i}$ in function of the parameter $c_{i}$. According to the conditions in (34), it is easy to show that $d_{i}$ and $e_{i}$ are defined with respect to $c_{i}$ like so

$$
\begin{aligned}
& d_{i}=m_{i}-\frac{2}{\tau_{*}} c_{i}, \\
& e_{i}=n_{i}+\frac{1}{\tau_{*}^{2}} c_{i},
\end{aligned}
$$

where $m_{i}$ and $n_{i}$ are expressed as follows:

$$
\begin{aligned}
& m_{i}=\frac{4}{\tau_{*}^{3}}\left(\theta_{i, *}^{-}-a_{i}-b_{i} \tau_{*}\right)-\frac{1}{\tau_{*}^{2}}\left(\dot{\theta}_{i, *}^{-}-b_{i}\right), \\
& n_{i}=-\frac{3}{\tau_{*}^{4}}\left(\theta_{i, *}^{-}-a_{i}-b_{i} \tau_{*}\right)+\frac{1}{\tau_{*}^{3}}\left(\dot{\theta}_{i, *}^{-}-b_{i}\right) .
\end{aligned}
$$

Using expression (32), the criterion $\mathscr{L}$ in (36) can be rewritten as follows:

$$
\mathscr{L}=\int_{0}^{\tau_{*}}\left(a+b t+c t^{2}+d t^{3}+e t^{4}\right)^{2} \mathrm{~d} t
$$

where $\quad a=a_{n s}+a_{s}+2 \varphi, \quad b=b_{n s}+b_{s}, \quad c=c_{n s}+c_{s}$, $d=d_{n s}+d_{s}$, and $e=e_{n s}+e_{s}$.
Relying on expressions (37)-(40), we obtain

$$
\begin{aligned}
& a=\theta_{n s, 0}+\theta_{s, 0}+2 \varphi, \\
& b=\dot{\theta}_{n s, 0}+\dot{\theta}_{s, 0}, \\
& d=m-\frac{2}{\tau_{*}} c, \\
& e=n+\frac{1}{\tau_{*}^{2}} c,
\end{aligned}
$$

where in (46) and (47), $m=m_{n s}+m_{s}$ and $n=n_{n s}+n_{s}$.

Relying on relations (41) and (42), we can easily demonstrate that the two parameters $m$ and $n$ are defined by the following expressions:

$$
\begin{aligned}
& m=-\frac{4}{\tau_{*}^{3}} a-\frac{1}{\tau_{*}^{2}}\left(D x_{*}^{-}+3 b\right), \\
& n=\frac{3}{\tau_{*}^{4}} a+\frac{1}{\tau_{*}^{3}}\left(D x_{*}^{-}+2 b\right),
\end{aligned}
$$

where $D=\left[\begin{array}{llll}0 & 0 & 1 & 1\end{array}\right]$.

Accordingly, the criterion $\mathscr{L}$ in (43) can be reformulated and rewritten under the following expression:

$$
\begin{aligned}
\mathscr{L}= & a^{2} \tau_{*}+a b \tau_{*}^{2}+\frac{1}{3}\left(b^{2}+2 a c\right) \tau_{*}^{3}+\frac{1}{2}(b c+a d) \tau_{*}^{4}+\frac{1}{5}\left(c^{2}+2 a e+2 b d\right) \tau_{*}^{5} \\
& +\frac{1}{3}(b e+c d) \tau_{*}^{6}+\frac{1}{7}\left(2 c e+d^{2}\right) \tau_{*}^{7}+\frac{1}{4} d e \tau_{*}^{8}+\frac{1}{9} e^{2} \tau_{*}^{9} .
\end{aligned}
$$

The criterion $\mathscr{L}$ in (50) is expression in function of the parameters $a, b, c, d$, and $e$, and also the nominal step period $\tau_{*}$ of the desired period-1 trajectory to be tracked. Thus, this quantity, that is, $\tau_{*}$, is predefined and then prefixed. The two parameters $a$ and $b$ depend on the initial state vector $x_{0}$ according to relations (44) and (45). The two parameters $d$ and $e$ depend, respectively, on the scalars $m$ and $n$, and also the design parameter $c$, which is unknown at this level. However, according to relations (48) and (49), the two scalars $m$ and $n$ are defined with respect to the desired state vector just before impact $x_{*}^{-}$, the nominal step period $\tau_{*}$, and the precomputed parameters $a$ and $b$. Thus, to solve this issue, we will substitute in the criterion $\mathscr{L}$ in (50), the two parameters $d$ and $e$ by their expressions (46) and (47). Accordingly, the criterion $\mathscr{L}$ will only depend on the unknown parameter $c$ to design in the sequel. Thus, it is straightforward to show that the criterion $\mathscr{L}$ in (43) is recast as the following second-order polynomial defined with respect to the design parameter $c$ :

$$
\mathscr{L}=\mu c^{2}+\eta c+\xi,
$$

with

$$
\begin{aligned}
& \mu=\frac{1}{630} \tau_{*}^{5}, \\
& \eta=\frac{1}{126} n \tau_{*}^{7}+\frac{1}{84} m \tau_{*}^{6}+\frac{1}{30} b \tau_{*}^{4}+\frac{1}{15} a \tau_{*}^{3}, \\
& \xi=a^{2} \tau_{*}+a b \tau_{*}^{2}+\frac{1}{3} b^{2} \tau_{*}^{3}+\frac{1}{2} a m \tau_{*}^{4}+\frac{2}{5}(a n+m b) \tau_{*}^{5}+\frac{1}{3} n b \tau_{*}^{6}+\frac{1}{7} m^{2} \tau_{*}^{7}+\frac{1}{4} m n \tau_{*}^{8}+\frac{1}{9} n^{2} \tau_{*}^{9} .
\end{aligned}
$$


By deriving with respect to $c$, it follows that the criterion $\mathscr{L}$ in (51) is minimum for the following optimal value of the parameter $c$ :

$$
c=-\frac{\eta}{2 \mu}
$$

As $c=c_{n s}+c_{s}$, then we can choose $c_{n s}$ and $c_{s}$ as follows:

$$
c_{n s}=c_{s}=-\frac{\eta}{4 \mu} \text {. }
$$

Remark 2. It is important to note that the two previous adopted control approaches are based on some features of the passive dynamic walking of the compass biped walker. The use of the passive gait in the control loop comes from the following fact:

(i) The passive compass biped robot presents a mechanically self-stabilized dynamic gait. Thus, the biped robot will try to stabilize its gait by itself.

(ii) The use of the passive dynamic walking allows to significantly increase the energy efficiency of the bipedal walking of the compass robot.

Thus, by considering these two motivations behind the use of the passive dynamic walking in the control loop of the compass biped robot, we employed in the first approach the whole passive trajectory during the swing phase. Thus, the biped robot will follow a passive trajectory that requires a zero energy. However, for the second control approach, which is based on the design of the 4 th-order polynomial trajectory, we will use two features of the passive gait, namely, the state vector just before impact $x_{*}^{-}$and the associated step period $\tau_{*}$. This methodology will at least allow to approach the passive gait.

\section{Simulation Results for the Control of Chaos}

Simulation results are presented and discussed in the following section to show through figures the effectiveness of proposed FF + PD control law (23) in the control of chaos and then in the stabilization of the compass-gait bipedal walker. We will show the results for the two control approaches. Moreover, the two gain matrices $K_{p}$ and $K_{v}$ in control law (23) are selected according to expressions in (26) and (27) to be such that $w_{1}=w_{2}=20$.

5.1. Simulation Results for the First Control Approach. In this part, we show the validity of proposed FF + PD control law (23) or, as discussed previously, PD control law (31), in the first control approach based on the passive dynamic walking. Thus, we will consider two cases with two different sets of the parameters $a$ and $\varphi$. The first case is for $\varphi=5.2^{\circ}$ and $a=0.5$, whereas the second one is for $\varphi=8.3^{\circ}$ and $a=0.8$. For these two cases, the passive gait of the compass bipedal robot is chaotic.

5.1.1. First Case: $\varphi=5.2^{\circ}$ and $a=0.5$. We consider the first case of a chaotic passive gait exhibited for the slope $\varphi=5.2^{\circ}$ and for the segment length $a=0.5$. The chaotic attractor is illustrated in Figure 5(b), inside which we have determined the period-1 unstable limit cycle defined by its unstable period-1 fixed point just after impact:

$$
x_{*}^{+}=\left[\begin{array}{llll}
-23.9357 & 13.5357 & -0.7928 & -67.1831
\end{array}\right] .
$$

The initial condition for the simulation is fixed at

$$
x_{0}=\left[\begin{array}{llll}
-10 & 5 & 50 & -10
\end{array}\right] \text {. }
$$

The simulation results of the tracking problem of the desired period-1 passive gait are given in Figure 8 . Figure 8(a) shows the variation of the controlled trajectory in the state space. The two red circles reveal the initial condition $x_{0}$, (56), from which the compass biped starts its walking. The large curves colored in cyan and green reveal the desired period-1 passive hybrid limit cycle. Obviously, the trajectory of the controlled gait converges to and remains on this desired limit cycle. Figure 8(b) shows the variation of the step period of the controlled gait. It is clear that after almost 6 steps, the gait converges to the desired one and also the obtained gait has the same desired step period. Figure 8(c) depicts the temporal variation of the control law $u$, which is composed of the two inputs $u_{s}$ and $u_{h}$. It is obvious that when the controlled gait tracked the desired one, the two controllers $u_{s}$ and $u_{h}$ are zero. Therefore, the controlled gait converges to the desired one and becomes entirely passive.

5.1.2. Second Case: $\varphi=8.3^{\circ}$ and $a=0.8$. We have realized also another simulation by adopting two different values of both $\varphi$ and $a$. Indeed, we have chosen a chaotic gait for $\varphi=$ $8.3^{\circ}$ and $a=0.8$. Recall that for such values of these two parameters $\varphi$ and $a$, the chaotic attractor was illustrated in Figure 7(b). For such adopted parameters of $\varphi$ and $a$, the period-1 fixed point just after impact of the unstable limit cycle is

$$
x_{*}^{+}=\left[\begin{array}{llll}
-28.1735 & 11.5735 & 240.6451 & -72.3556
\end{array}\right] .
$$

The simulation results are given in Figure 9. It is obvious that the passive gait is controlled and stabilized at the desired period-1 hybrid limit cycle. When the tracking is realized, and relying on Figure 9(c), the control law $u$ becomes zero and hence the gait of the compass biped walker becomes passive.

To check the efficiency of the method, we simulated the solution of impulsive hybrid nonlinear dynamics 10(a) and 10(b) using the Poincaré section $\Gamma$ defined in (11). The simulation results are illustrated in Figure 10 without and with the control for $\varphi=8.3^{\circ}$ and $a=0.8$. Without control, we observe in Figure 10(a) an infinity of points which indicates a chaotic behavior. This phenomenon disappears after applying the control based on the passive dynamic walking as it is shown in Figure 10(b) where it is presented only one single point of intersection with the Poincaré section $\Gamma$.

5.1.3. Further Simulation Results for Tracking the Passive Gait with $\varphi=5.2^{\circ}$ and $a=0.5$. In this section, we will propose some additional simulation results showing the effectiveness 


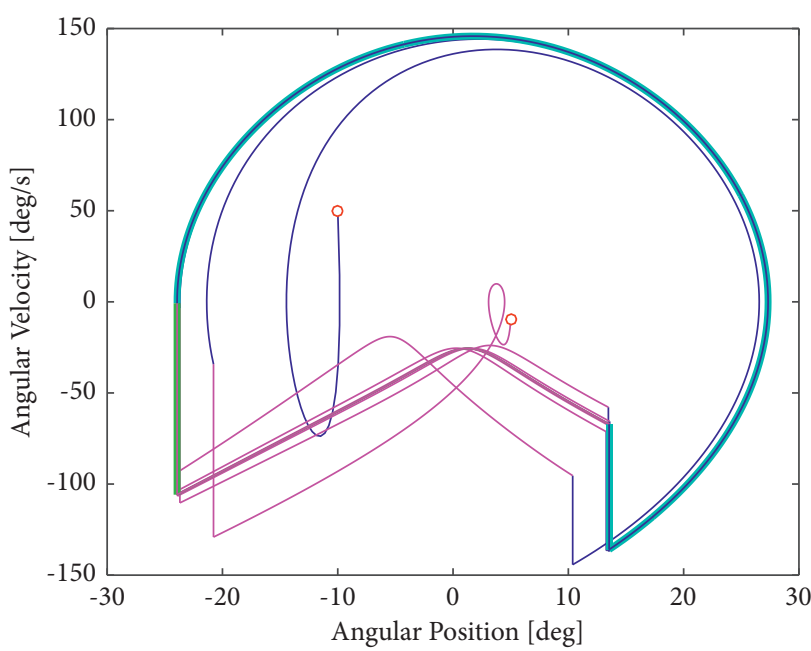

(a)

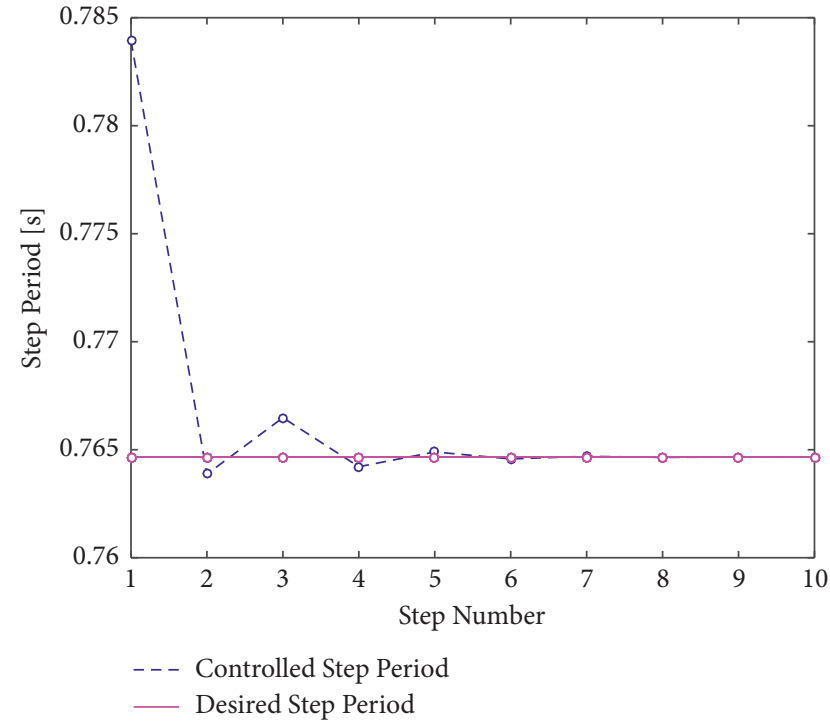

(b)

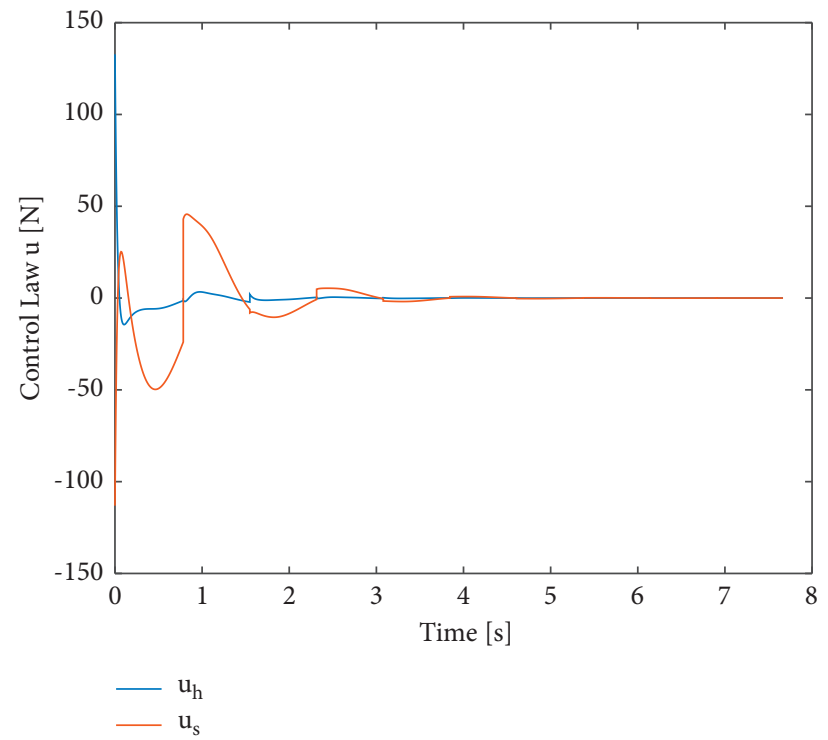

(c)

Figure 8: Control of the chaotic passive gait of the compass biped walker for the parameters $a=0.5$ and $\varphi=5.2^{\circ}$, using the passive dynamic walking-based control approach.

of the adopted control law in the tracking of the designed period-1 trajectory (period-1 passive gait). We will considered the desired period-1 passive trajectory to be that generated by the compass-gait biped walker during the swing phase and for the nominal parameters $\varphi=5.2^{\circ}$ and $a=0.5$. Thus, the nominal initial condition is given by the state vector $x_{*}^{+}$defined in (55). In addition, we propose to vary the slope angle to a different value and hence the compass biped robot walks down a slope different to the desired one. We will take four cases: $\varphi=7^{\circ}, \varphi=10^{\circ}, \varphi=0^{\circ}$, and $\varphi=-5^{\circ}$. It is important to note that for each slope, the desired period-1 passive gait is always the same. Then, the objective is to track the same period-1 trajectory. Notice that for the case $\varphi=0^{\circ}$, the biped robot walks on a level ground. However, for the case where the slope is negative, and then for the fourth case $\varphi=-5^{\circ}$, the compass robot goes on an inclined surface compared to the other cases where the biped robot descends the inclined walking surface. It is important to note that for these four different slopes, the passive compass walker cannot experience a successful bipedal walking on the walking surface. Emanated from any initial condition, the biped robot falls down after some few steps. We note that the initial condition for the simulation of the compass biped robot is adopted to be $x_{0}$ given by (56).

(1) Bipedal Walking for the Slope. $\varphi=7^{\circ}$. Figure 11 shows the simulation results for the first slope $\varphi=7^{\circ}$. Figure 11(a) reveals the desired period- 1 passive limit cycle (formed by the green and cyan large curves) and the controlled trajectory of the biped robot emanated from the initial 


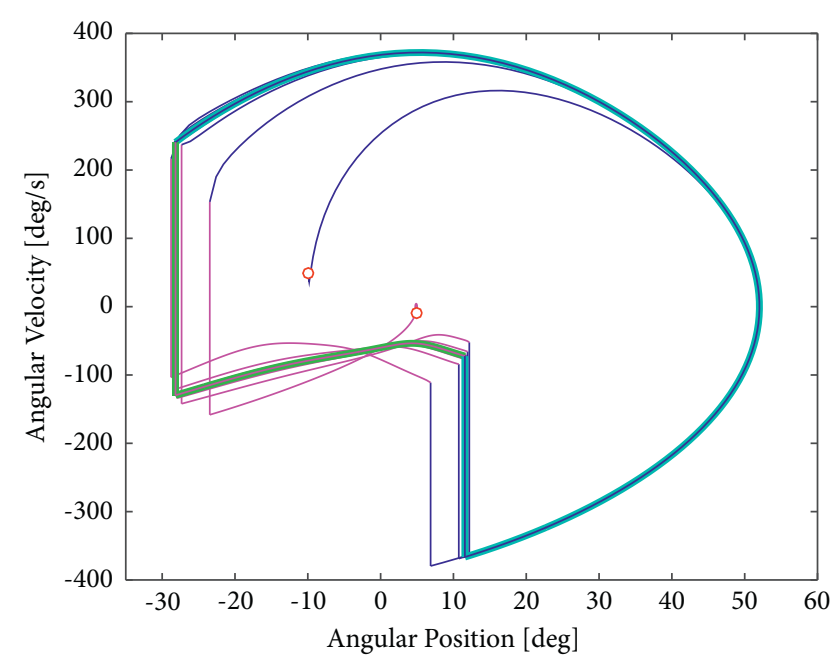

(a)

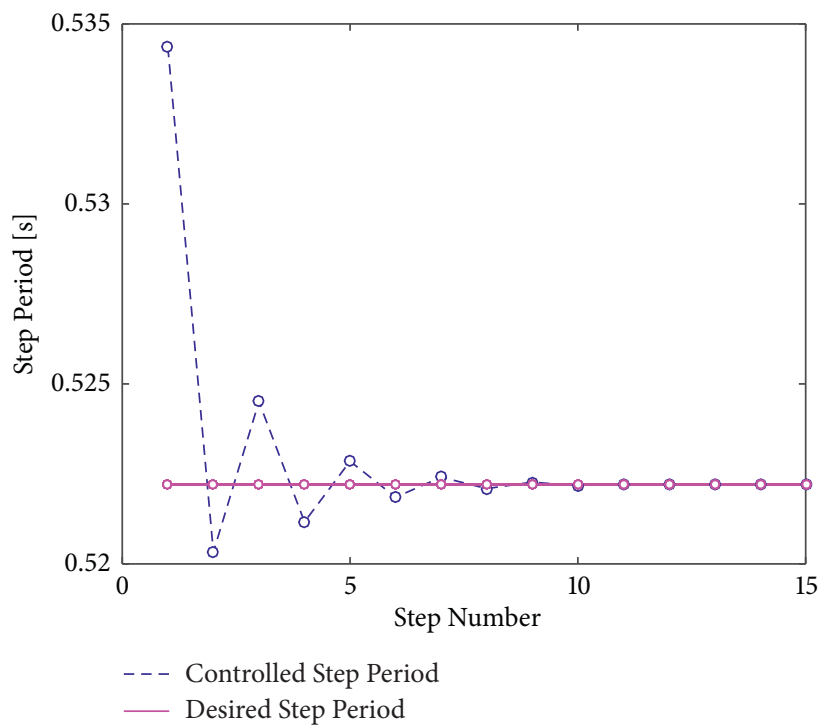

(b)

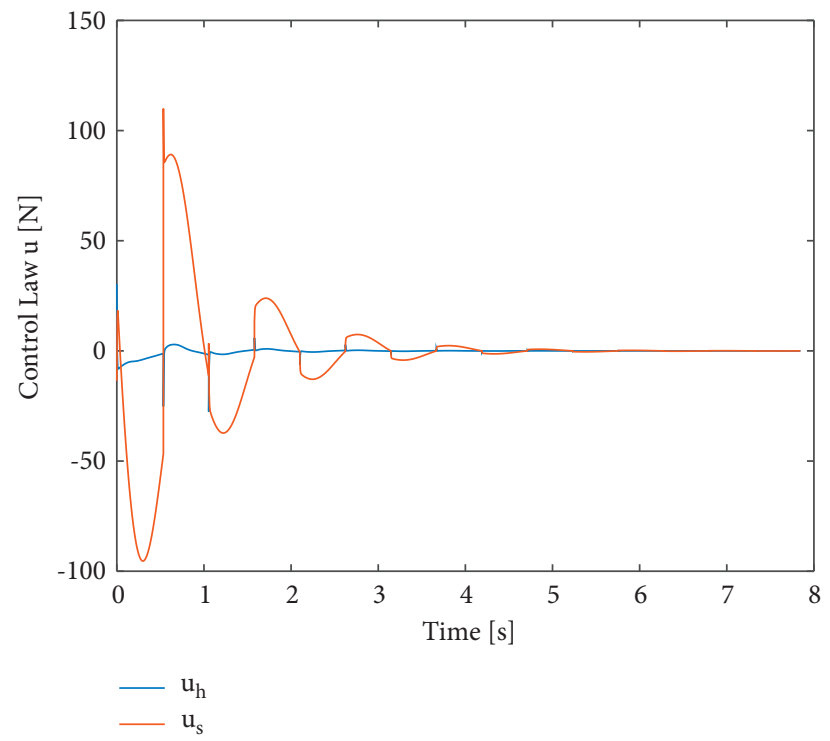

(c)

Figure 9: Simulation results showing the controlled chaotic gait of the compass-type biped walker for $\varphi=8.3^{\circ}$ and $a=0.8$ and using the first control approach based on the passive dynamic walking.

condition $x_{0}$. It is worth to note that by increasing the slope angle, the passive and then the uncontrolled compass-gait biped robot needs a bit more time to reach the walking surface. Therefore, the state vector just before impact associated to the slope $\varphi=5.2^{\circ}$, saying $x_{*, \varphi=5.2^{\circ}}^{-}$, is completely different to the state vector just before impact associated to the slope $\varphi=7^{\circ}$, that is, $x_{*, \varphi=7^{\circ}}^{-}$. Nevertheless, as the predefined objective is to track the desired period-1 passive gait generated for the slope $\varphi=5.2^{\circ}$, and since the compass biped robot walks down the slope $\varphi=7^{\circ}$, the period-1 passive gait will be re-emanated just after the impact from the state vector $x_{*, \varphi=5.2^{\circ}}^{+}$and not from the state vector $x_{*, \varphi=7^{\circ}}^{+}$, as described previously in the algebraic equation in (30). This methodology will allow to generate always the desired period-1 (unstable) passive gait.
We can also observe clearly the tracking of the desired trajectory from Figures 11(c)-11(f), where Figures 11(c) and 11(d) (resp., Figures 11(e) and 11(f)) show the in-time trajectory of the angular position and the angular velocity of the swing leg (resp., the stance leg). The tracking of the desired period-1 passive gait is obvious. It is clear from these two figures, Figures 11(c) and 11(d), that just after the impact of the swing leg with ground, the two trajectories (the desired gait and the controlled one) are different since they start from two different initial conditions, as explained previously. However, and via adopted control law (31), the compass robot follows the desired period-1 passive gait. Figure 11(b) reveals the applied control law $u$, defined by expression (31). It seems that the biped robot requires some nonnegligible energy to track the desired period-1 passive 


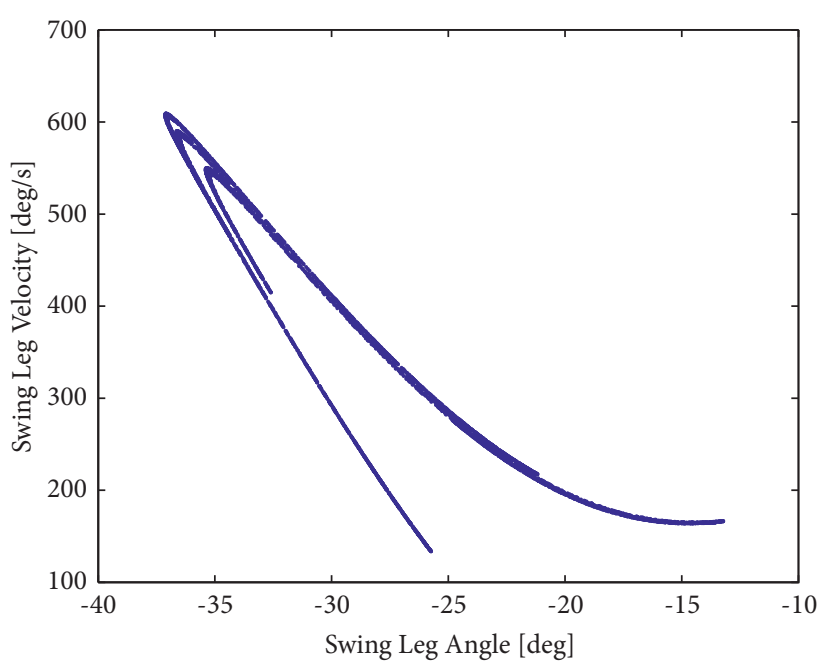

(a)

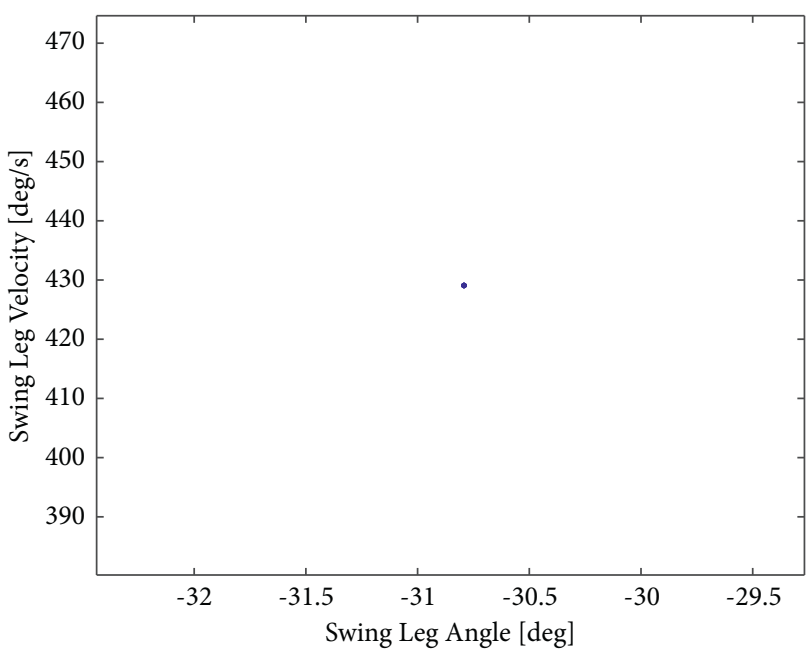

(b)

FIgURe 10: Simulation results showing the uncontrolled (a) and the controlled (b) passive gait of the compass-type biped walker for $\varphi=8.3^{\circ}$ and $a=0.8$ using the Poincaré section $\Gamma$ defined in (11).

gait. When the bipedal walking is controlled, the control effort applied at the hip, $u_{h}$, converges to zero. Moreover, the stance leg's controller $h_{s}$ decreases along the swing phase. Nevertheless, as this phase ends and the two initial conditions of the two trajectories become different at the beginning of a new walking step, the control effort increases again. This scheme continues for every step.

(2) Bipedal Walking for the Slope. $\varphi=10^{\circ}$. We consider now a different slope angle $\varphi=10^{\circ}$, on which the compass biped robot walks down. The desired period-1 passive gait is that generated for the slope $\varphi=5.2^{\circ}$. The simulation results are illustrated in Figure 12. In Figure 12(a), we observe the tracking of the desired period- 1 passive gait during the swing phase. The biped robot requires some milliseconds to follow the desired trajectory. The temporal evolution of the angular position and the angular velocity of the swing leg, respectively, in Figures $12(\mathrm{c})$ and $12(\mathrm{~d})$, and that of the stance leg, respectively, in Figures 12(e) and 12(f), confirm this fact. As noted previously, at the beginning of the swing phase, the desired passive trajectory and the controlled one are completely different. This difference requires a control effort to allow the compass walker tracks the desired passive gait. Figure 12(b) reveals the temporal evolution of the applied control law $u$. Compared to Figure 11(b), the control effort is increased.

In Figures 11 and 12, the tracking of the desired passive gait of the stance leg (Figures 11(e) and 11(f) for $\varphi=7^{\circ}$, and Figures 12(e) and 12(f) for $\varphi=10^{\circ}$ ), is not completed and requires more time. However, this fact cannot happen since the duration of the swing phase is constraint by the impact of the swing leg with the walking surface and then limited by the time of the walking step. To overcome this problem, the solution is to increase the values of the two gains $K_{p}$ and $K_{v}$ of control law (23). Thus, in (26) and (27), we choose $w_{1}=w_{2}=40$. Figure 13 reveals the simulation results with these new matrix gains $K_{p}$ and $K_{v}$. Compared to Figure 12(a), the shape of the controlled attractor (period-1 limit cycle) in Figure 13(a) is changed. Furthermore, the temporal evolution of the four states in Figures 13(c)-13(f) shows the tracking of the desired period-1 passive gait of the compass biped robot better than that in Figure 12 by adopting $w_{1}=w_{2}=20$. However, as seen in Figure 13(b), the increase of $w_{1}$ and $w_{2}=20$ and then of the two gains $K_{p}$ and $K_{v}$ of the PD control part provokes a considerable increase of the control effort applied to the biped robot.

(3) Bipedal Walking on a Level Ground, for the Slope. $\varphi=0^{\circ}$. We choose now the slope angle $\varphi=0^{\circ}$ and then the compass walker walks on a level ground. We reselect $w_{1}=w_{2}=20$ for the feedback gains $K_{p}$ and $K_{v}$ of adopted FF + PD control law (23). Figure 14 shows the simulation results. Compared to the previous cases for $\varphi=7^{\circ}$ and $\varphi=10^{\circ}$, the swing leg of the compass robot needs a smaller time to encounter the walking surface. Hence, the step period of the bipedal walking for the slope $\varphi=0^{\circ}$ is shorter than that for $\varphi=5.2^{\circ}$. This fact can be observed for the passive and controlled attractors in Figure 14(a). Indeed, and compared to the previous cases, the state vector just after the impact of the desired period-1 passive attractor (color in green and cyan) is located at the left side with respect to the state vector just before the impact.

Figures 14(c)-14(e) and Figure 14(e) show the curves of the angular positions and the angular velocities of the two legs of the compass bipedal walker. The tracking of the desired trajectory is obvious. In addition, Figure 14(b) reveals the control effort $u$ applied to the biped robot while walking. As in the previous cases, the hip controller $u_{h}$ requires small energy compared to the stance leg's controller $u_{s}$.

(4) Bipedal Walking Ascending the Slope. $\varphi=-5^{\circ}$. In this last case, we consider a negative slope angle, $\varphi=-5^{\circ}$. Thus, the compass biped walker ascends an inclined plane. Recall that the desired period-1 passive gait to be tracked by the compass robot is generated for the slope $\varphi=5.2^{\circ}$. The simulation results are illustrated in Figure 15. Figure 15(a) 

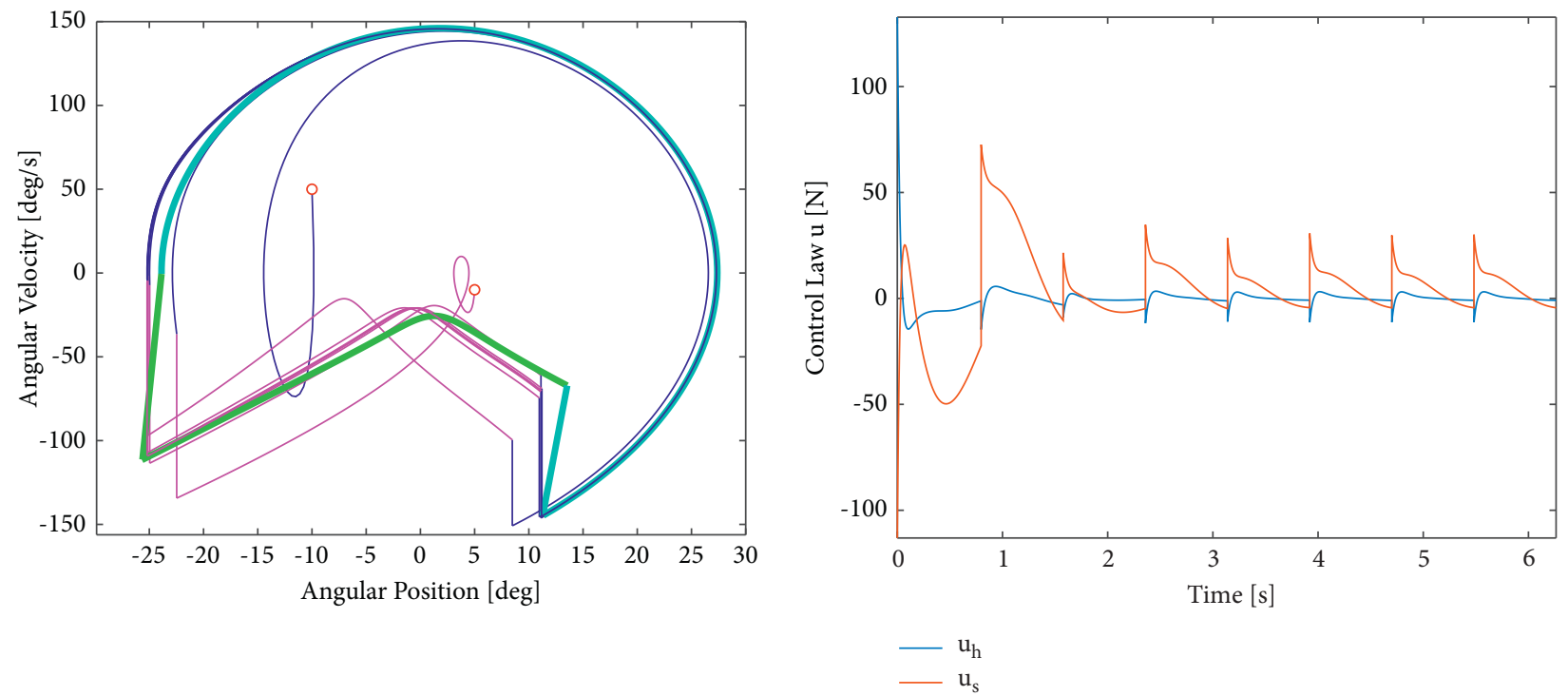

(a)
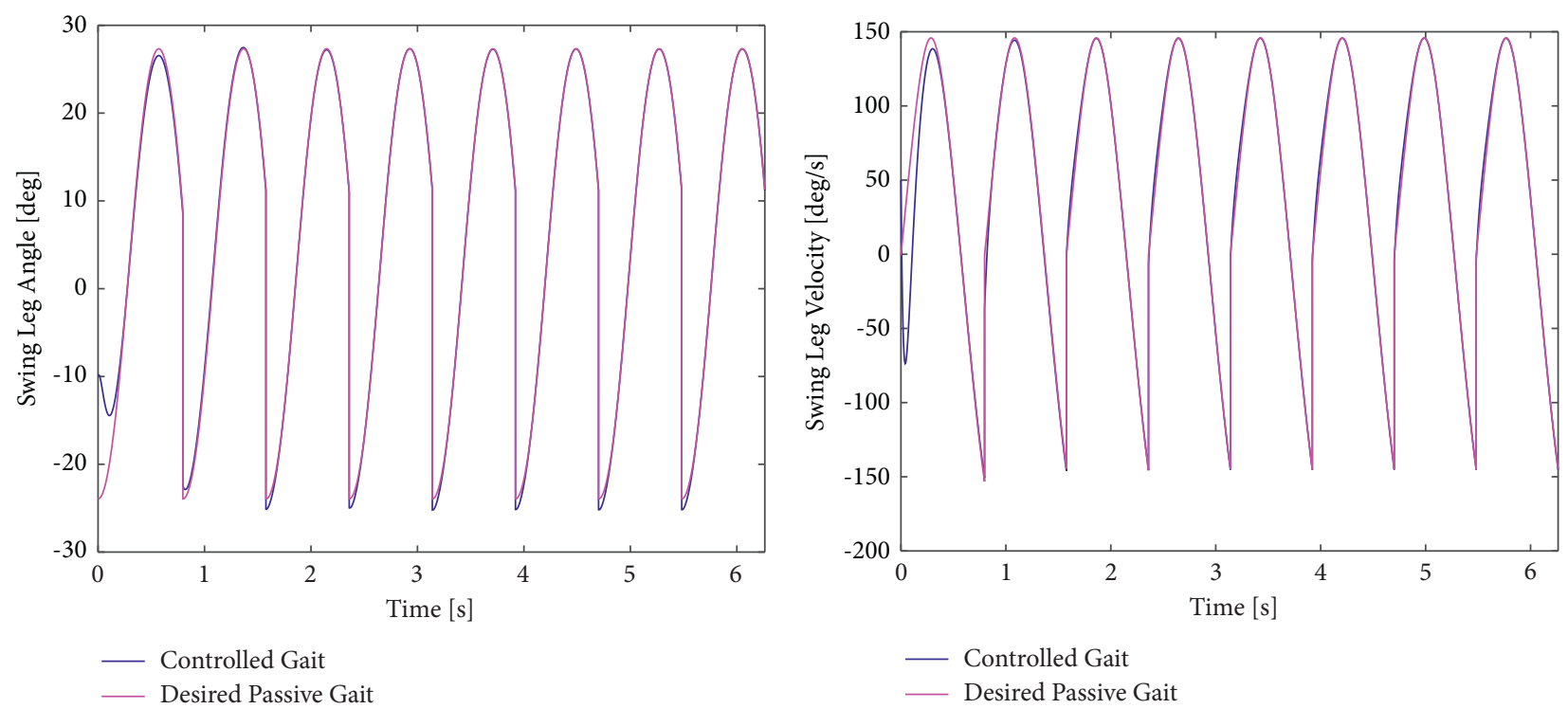

(c)

(d)

Figure 11: Continued. 


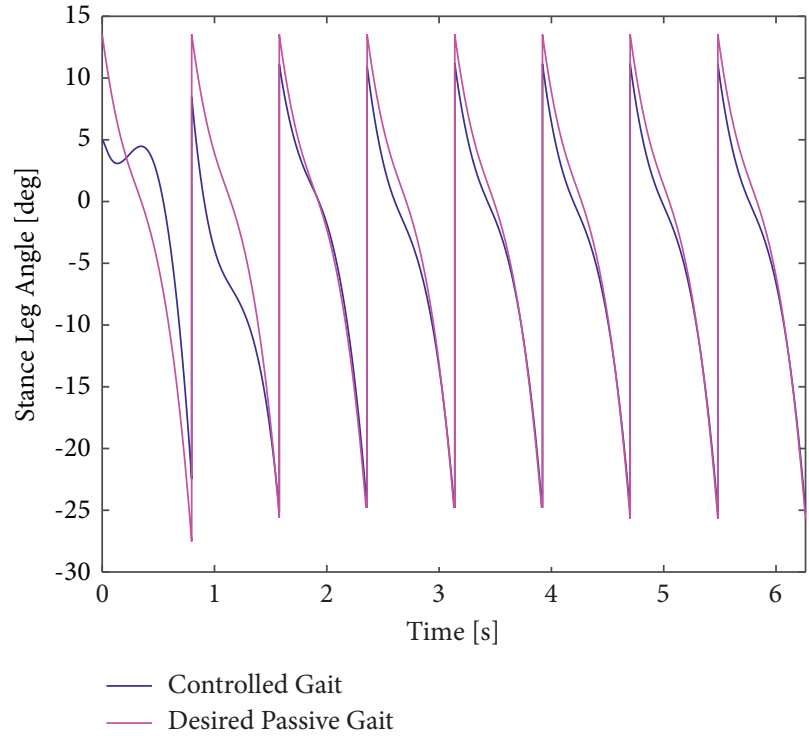

(e)

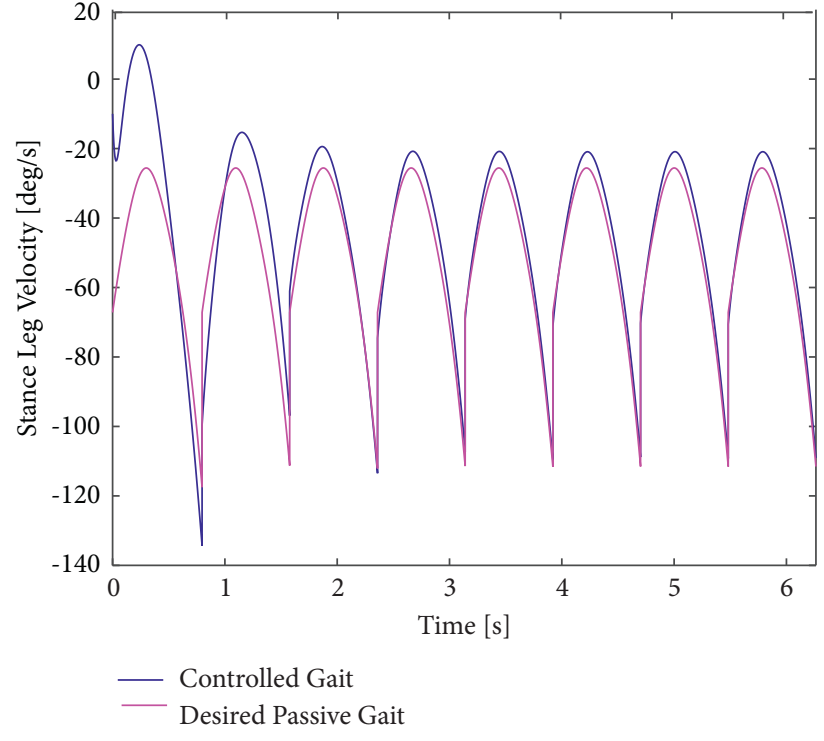

(f)

FIGURE 11: Simulation results revealing the tracking of the desired period-1 passive gait of the compass-type biped walker for $\varphi=7^{\circ}$ and $a=0.5$. Here, the desired period-1 passive dynamic walking is generated for the slope $\varphi=5.2^{\circ}$. In addition, we selected $w_{1}=w_{2}=20$ for the two feedback gains $K_{p}$ and $K_{v}$ in (26) and (27) of adopted control law (23).

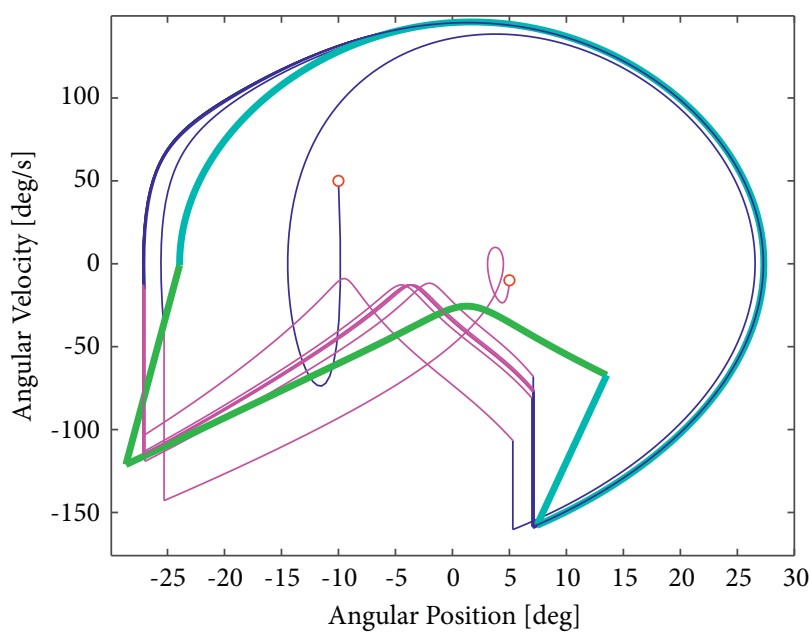

(a)

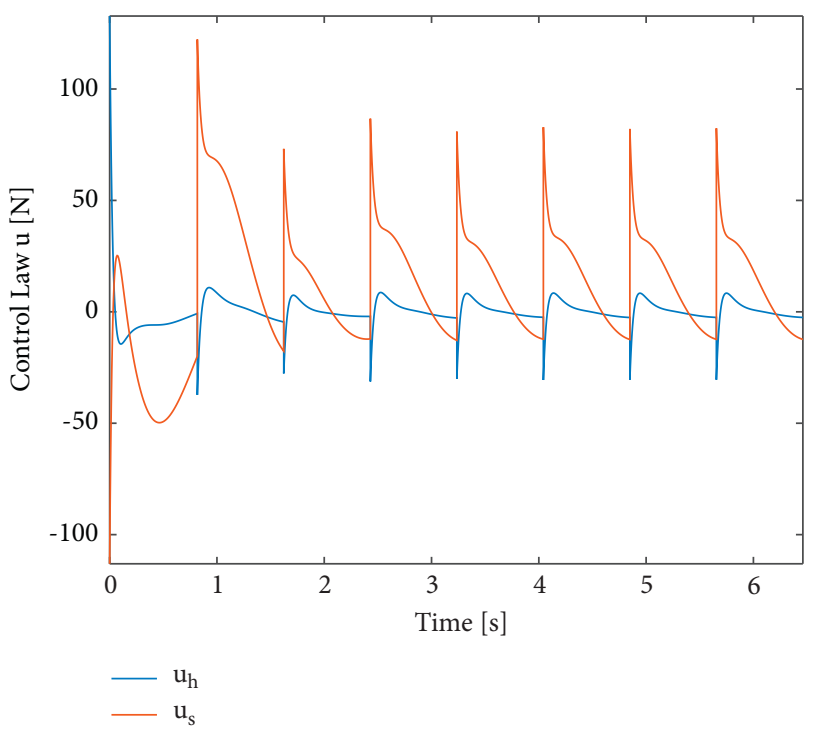

(b)

Figure 12: Continued. 


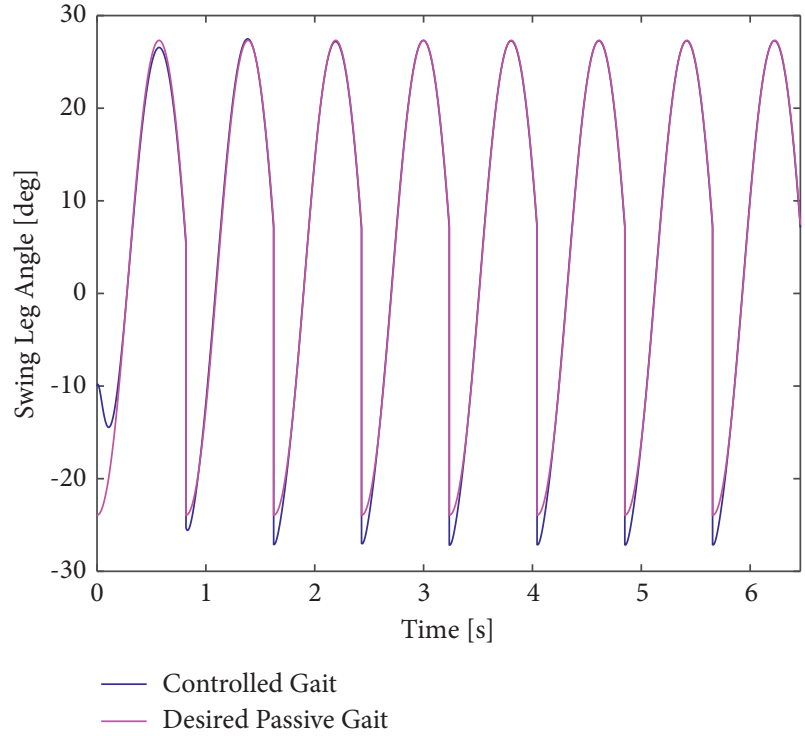

(c)

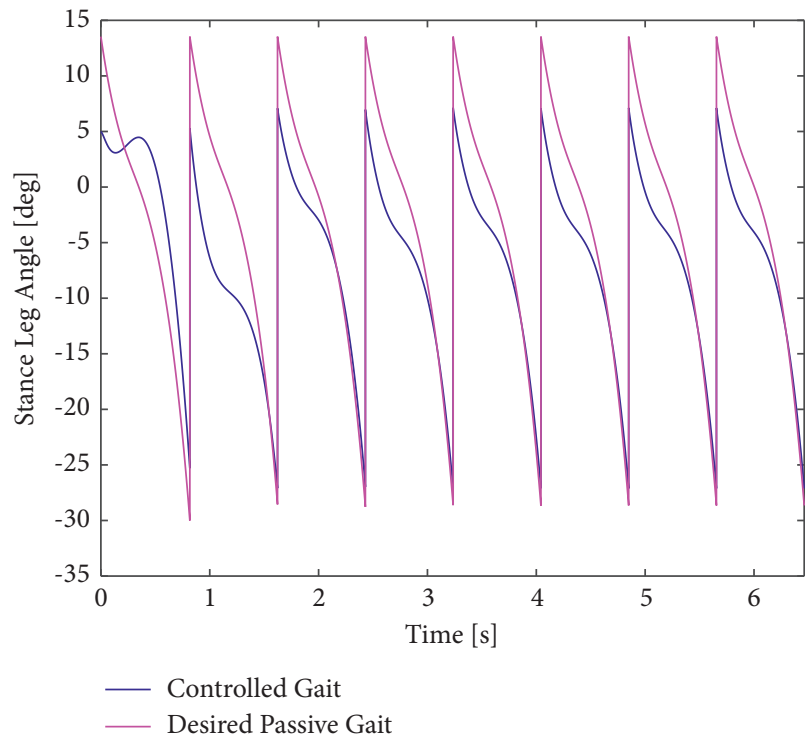

(e)

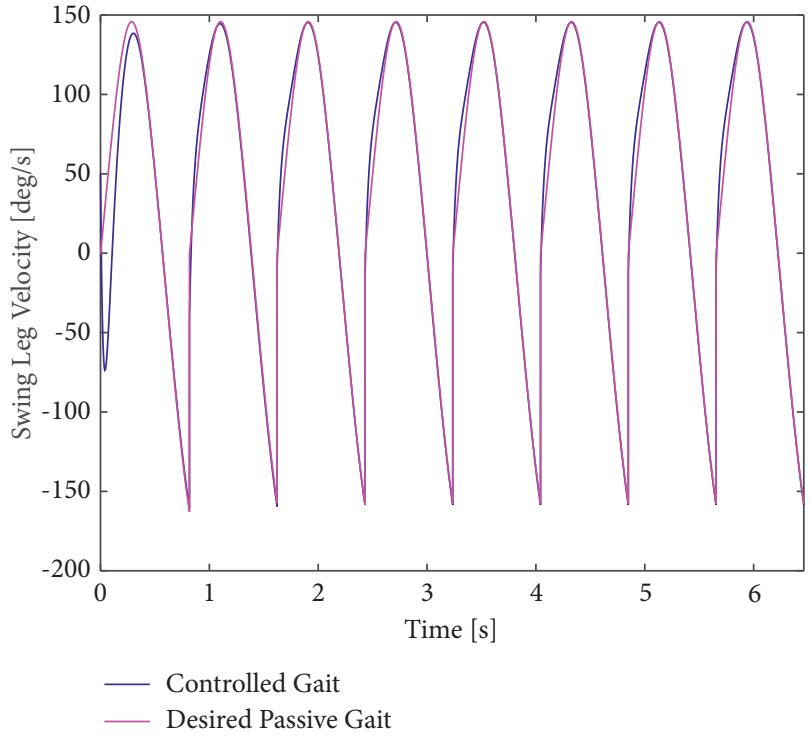

(d)

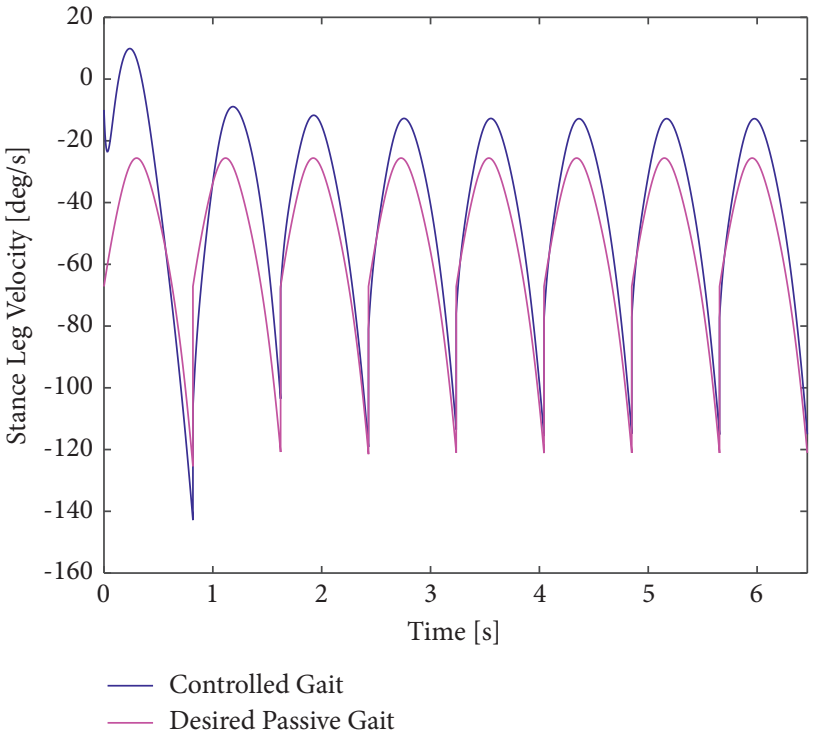

(f)

FIGURE 12: Simulation results showing the tracking of the desired period-1 passive gait of the compass-gait bipedal walking robot for $\varphi=10^{\circ}$ and $a=0.5$, and for $K_{p}$ and $K_{v}$ in (26) and (27) of control law (23) with $w_{1}=w_{2}=20$. As previously, the desired period-1 passive gait is generated for the slope $\varphi=5.2^{\circ}$.

shows the controlled attractor and the desired period-1 passive hybrid limit cycle to track by the compass robot. Figure 15(b) reveals the control effort applied to the biped robot while ascending the inclined plane. Compared to the case $\varphi=0^{\circ}$ and then Figure 14(b), the biped walker needs more control effort to track the desired trajectory and then to walk on the walking surface. Figures 15(c)-15(f) show the temporal evolution of the angular positions and the angular velocities of the two legs. Obviously, the swing leg tracks the desired trajectory. However, for the stance leg, there is a clear gap between the desired trajectory and the controlled one. Nevertheless, the angular position of the stance leg reaches the desired trajectory at the end of the swing phase. It seems that the stance leg needs more time to track the desired passive gait. However, as noted previously, the tracking procedure is restricted by the period of the swing phase and hence of the bipedal walking. In order to improve the tracking scheme, and as we have realized previously in the second case for $\varphi=10^{\circ}$, it is possible to increase the value of $w_{1}$ and $w_{2}$ and then of the two feedback gains $K_{p}$ and $K_{v}$ in (26) and (27) of control law (23).

5.1.4. Further Simulation Results for Tracking the Passive Walk with $\varphi=8.3^{\circ}$ and $a=0.8$. As the previous part, the objective here is to track the passive dynamic walking of the 

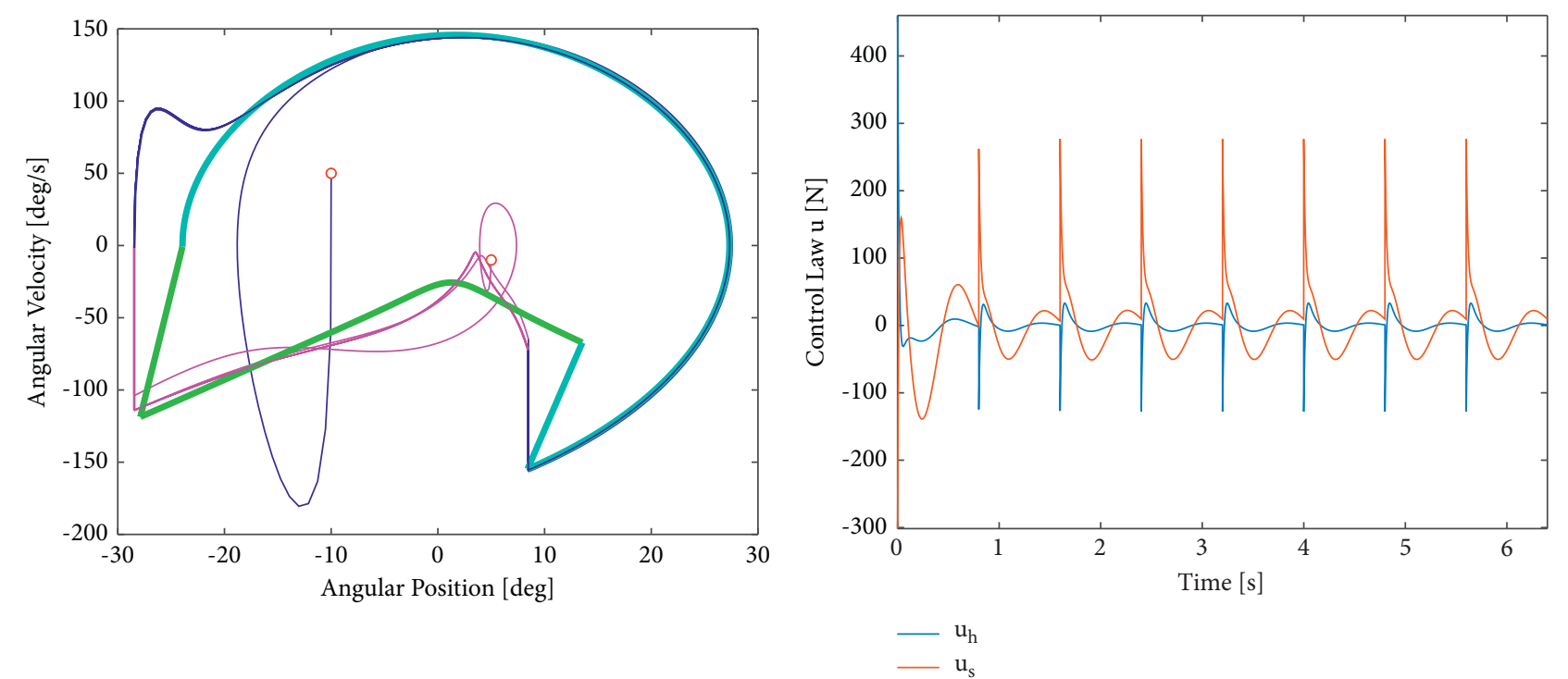

(a)
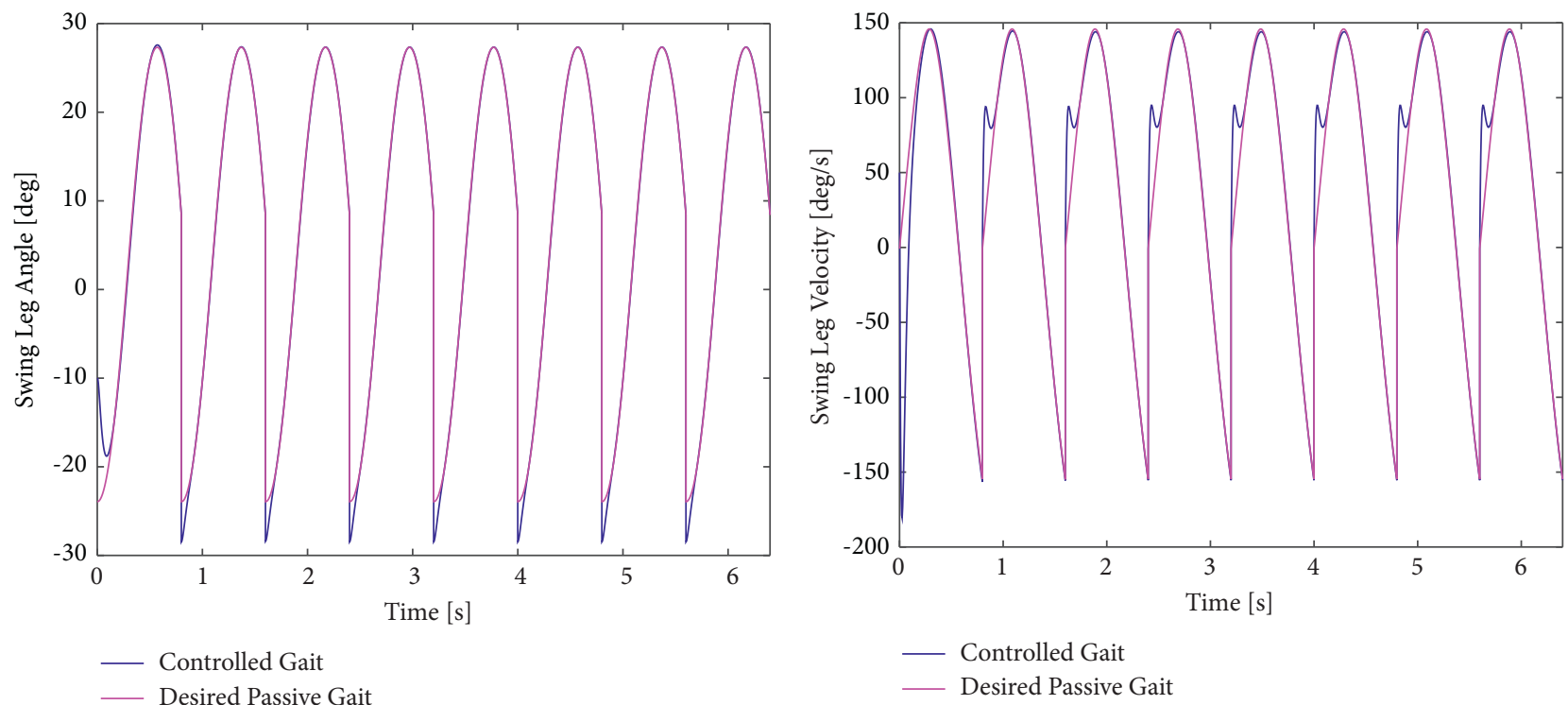

(c)

(d)

FIGURE 13: Continued. 


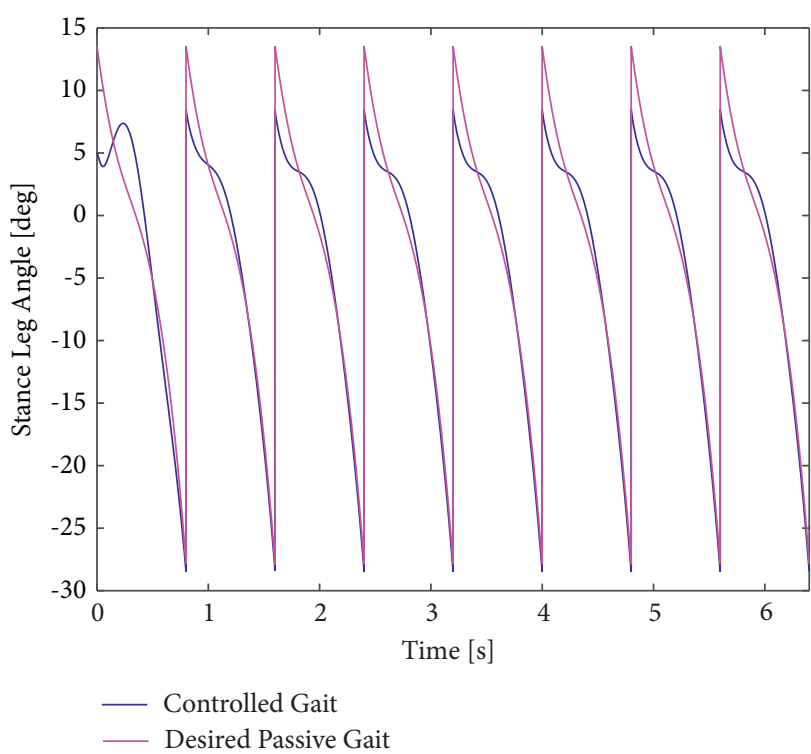

(e)

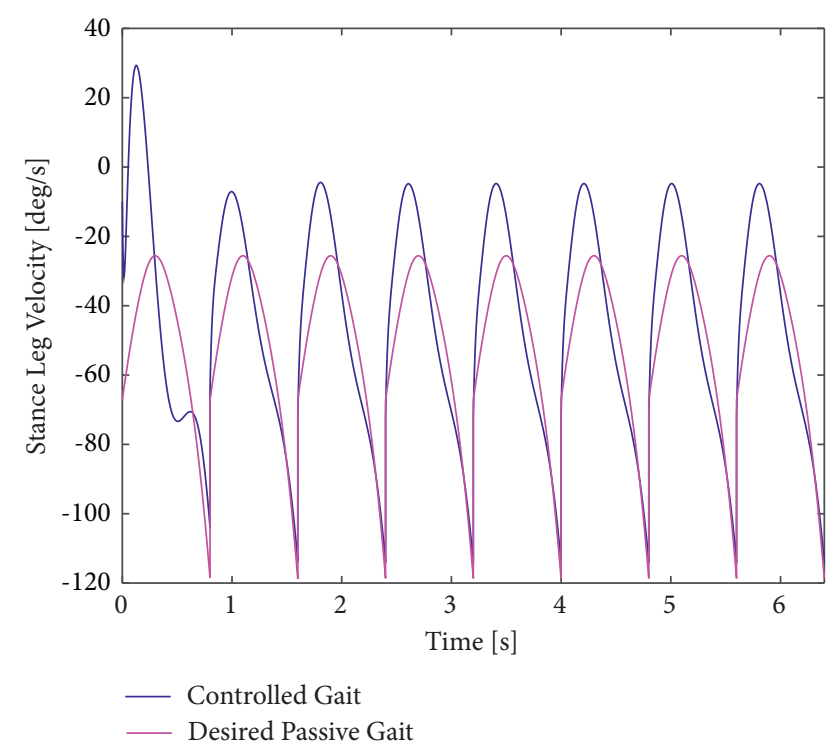

(f)

Figure 13: Simulation results showing the tracking of the desired period-1 passive gait of the bipedal compass $\operatorname{robot}$ for $\varphi=10^{\circ}$ and $a=0.5$. The two matrix gains $K_{p}$ and $K_{v}$ in (26) and (27) of adopted control law (23) are fixed such that $w_{1}=w_{2}=40$. Moreover, the desired period1 passive walking to be tracked is generated for the slope $\varphi=5.2^{\circ}$.

compass-gait bipedal robot generated for a different set of parameters $\varphi$ and $a$, namely, $\varphi=8.3^{\circ}$ and $a=0.8$. Actually, we have obtained almost the same simulation results and remarks. Thus, we will consider two cases: $\varphi=15^{\circ}$ and $\varphi=0^{\circ}$. The first case $\varphi=15^{\circ}$ is for steeper slope, whereas the second case $\varphi=0^{\circ}$ corresponds to a bipedal walking on a level ground. The initial condition for the simulation of the compass robot is fixed as previously to be $x_{0}$ given by (56).

(1) Bipedal Walking down the Slope. $\varphi=15^{\circ}$. Let us consider the first case of the slope $\varphi=15^{\circ}$. Thus, the compass robot will walk down an inclined plane of slope $\varphi=15^{\circ}$ and will track the desired period-1 passive gait generated for the nominal slope $\varphi=8.3^{\circ}$. Figure 16 shows the obtained simulation results. Figure 16(a) reveals the controlled attractor approaching the desired period-1 passive limit cycle. Figure 16(c)-16(f) confirm the tracking scheme. As previously, the stance leg needs more control effort to efficiently track the desired passive gait. Figure 16(b) reveals the control effort applied to the biped robot to track the desired period-1 passive gait. Like previous studies, the stance leg needs an important control effort, $u_{s}$, to track the desired gait. However, the control effort applied at the hip, $u_{h}$, is low.

(2) Bipedal Walking on the Level Ground. $\varphi=0^{\circ}$. In this part, the compass-gait biped robot under adopted control law (23) walks on the level ground and then the slope angle of the inclined surface is $\varphi=0^{\circ}$. The desired period-1 passive gait to be tracked is generated for the nominal slope $\varphi=8.3^{\circ}$ and for $a=0.8$. Simulation results are illustrated in Figure 17. It is obvious that the controlled gait tracks the desired period-1 passive gait. Figure 17(b) reveals the control effort $u$ applied to the biped robot while walking on the level ground. As previously, the stance leg needs an important control level to track the desired trajectory.

5.2. Simulation Results for the Second Control Approach. In this section, we present the simulation results for the second control approach by tracking a 4th-order Spline trajectory, which was designed in Section 4.3.2. Recall that the control law is FF+ PD controller (23). For such controller, we have adopted the same gain matrices $K_{p}$ and $K_{v}$ in (26) and (27). In addition, for the simulation of the controlled behavior, we have selected the same initial condition $x_{0}$ taken in the previous control approach based on the passive dynamic walking, that is, the state $x_{0}$ in (56). Moreover, we have considered the two same cases of the parameters $a$ and $\varphi$.

It is important to mention that we have achieved several simulations with different values of $K_{p}$ and $K_{v}$ and we have found (almost) the same behavior of the controlled passive gaits. We have selected, for example, $K_{p}=0$ and $K_{v}=0$ and as result, and we obtained the same control picture. Notice that with these values of the matrix gains $K_{p}$ and $K_{v}$, the PD action in FF + PD control law (23) is canceled, $u_{\mathrm{PD}}=0$, and then such control law becomes feedforward controller $u_{\mathrm{FF}}$ (23). It is worth noting that such controller has interesting advantages that it offers in implementation compared to the $\mathrm{PD}$ controller $u_{\mathrm{PD}}$ that was used in the previous analysis, and also compared to FF + PD control law $u_{\mathrm{FF}+\mathrm{PD}}$ (23). Indeed, the controller $u_{\mathrm{FF}}$ does not depend on the angular position $q$ nor on the angular velocity $\dot{q}$ of the biped robot. Moreover, such controller $u_{\mathrm{FF}}$ does not possess any design parameter. Therefore, the control process of the compass-gait walker 

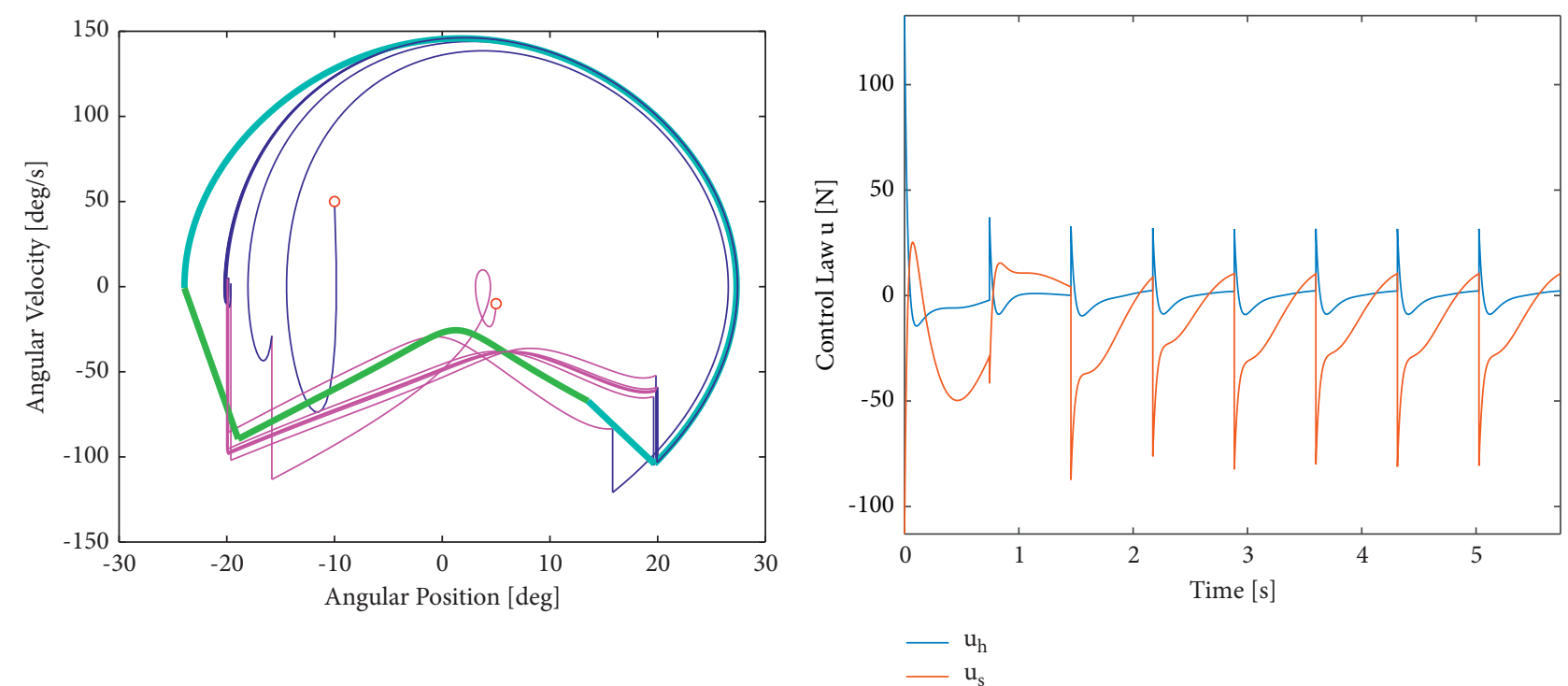

(a)

(b)
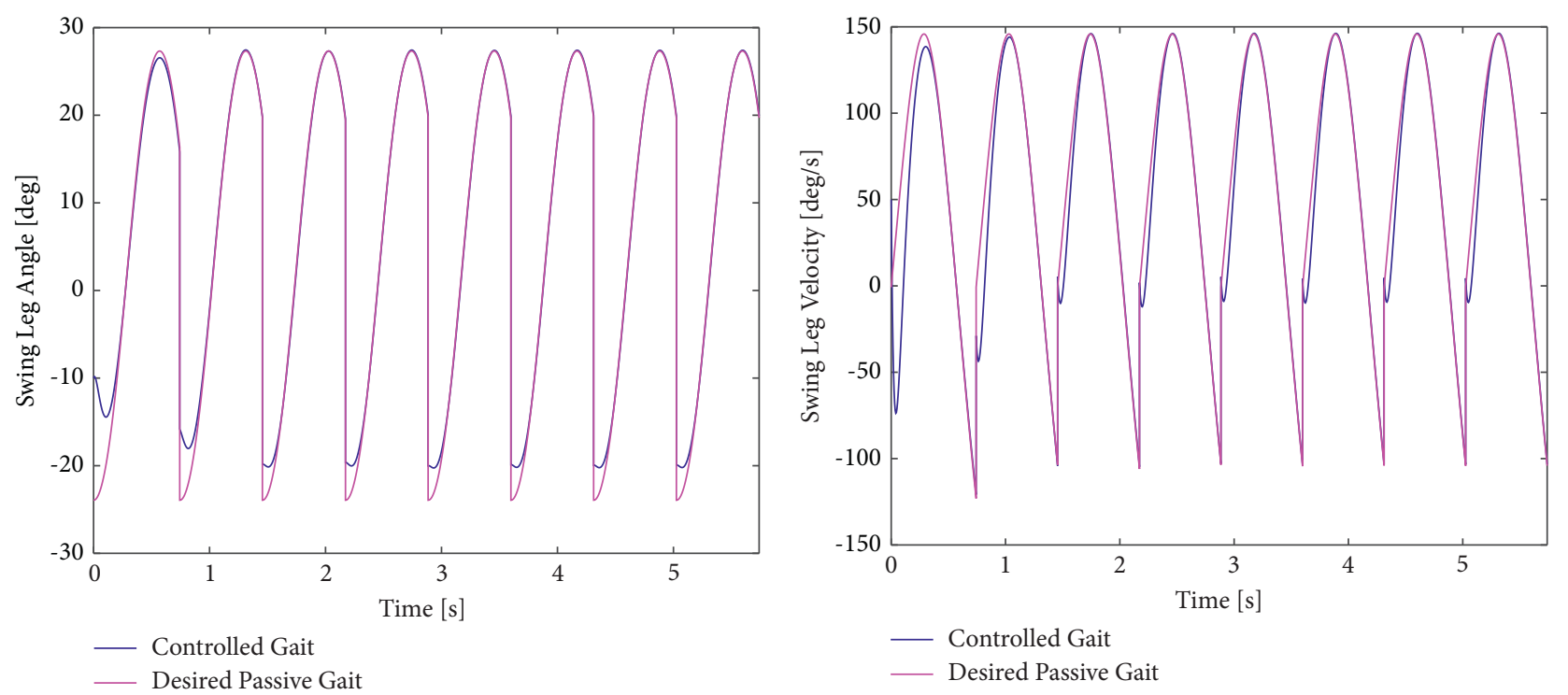

(c)

(d)

Figure 14: Continued. 


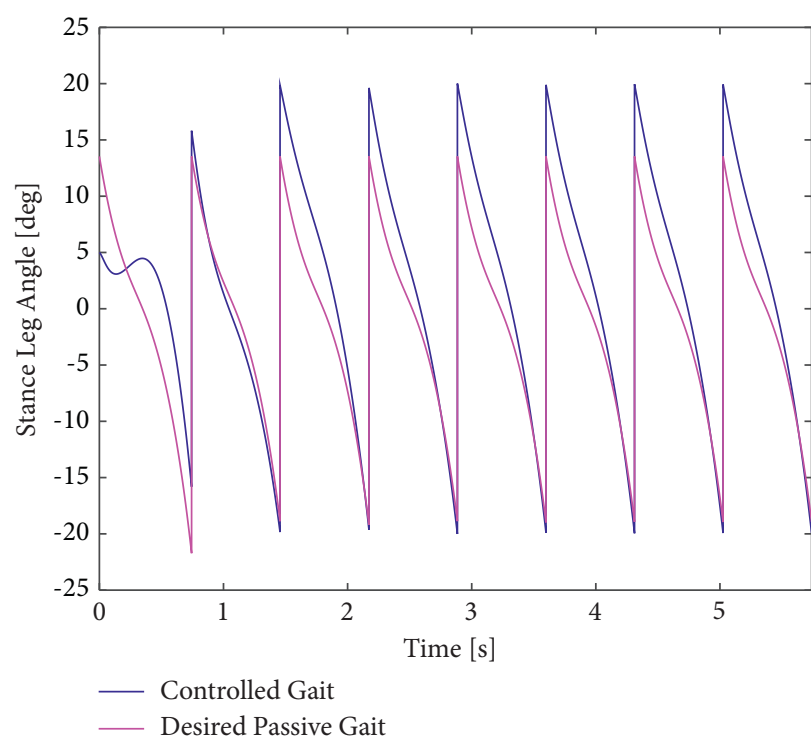

(e)

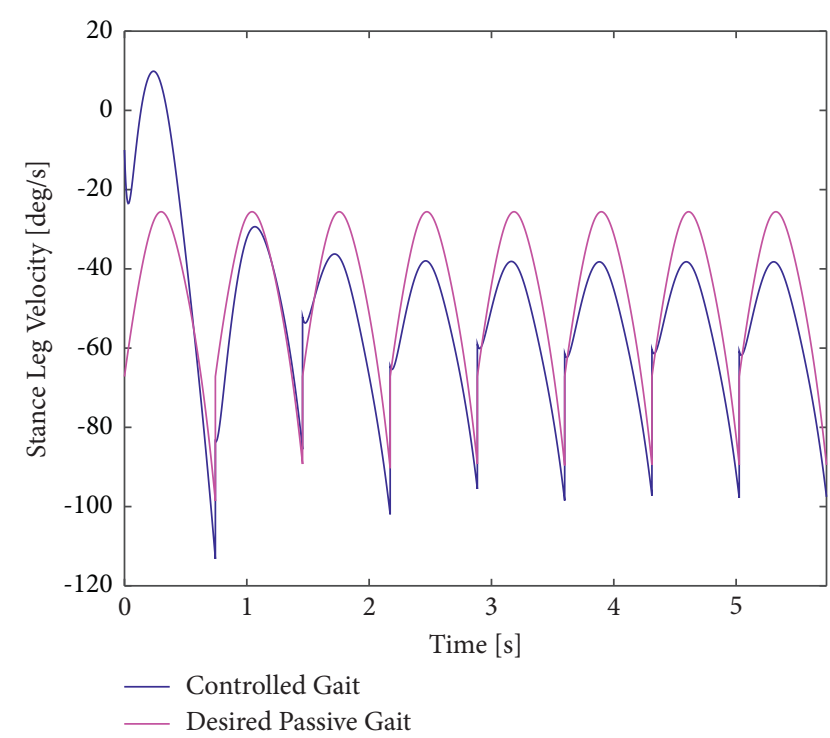

(f)

FIGURE 14: Simulation results showing the tracking of the desired period-1 passive gait of the compass walker for $\varphi=0^{\circ}$ and $a=0.5$, and for $K_{p}$ and $K_{v}$ in (26) and (27) of adopted FF + PD control law (23) with $w_{1}=w_{2}=20$. The desired period-1 passive gait is obtained for the slope $\varphi=5.2^{\circ}$.

using this second approach and also using the FF control law $u_{\mathrm{FF}}$ does not require the use of sensors to measure the angular quantities $q$ and $\dot{q}$. One only needs to design and compute the desired trajectory $q_{d}(t)$ and hence $\dot{q}_{d}(t)$ and $\ddot{q}_{d}(t)$ (maybe off-line).

5.2.1. First Case: $\varphi=5.2^{\circ}$ and $a=0.5$. Let start with the first simulation results by considering the case of the chaotic passive gait for $a=0.5$ and $\varphi=5.2^{\circ}$. Recall that the chaotic attractor generated using these values of $a$ and $\varphi$ is illustrated in Figure 5(b). As noted previously, the design of the constants $a_{i}, b_{i}, c_{i}, d_{i}$, and $e_{i}$, for $i=\{n s, s\}$, depends on the desired step period $\tau_{*}$ of the period-1 passive gait. Thus, for such values of $a$ and $\varphi$, we have $\tau_{*}=0.7647$.

Figure 18 shows the simulation results for this first case, that is, for $a=0.5$ and $\varphi=5.2^{\circ}$. It is obvious from Figure 18(a) that, starting from the departure point $x_{0}$ (marked with red circles), the trajectory converges rapidly to the planned period-1 gait. Initially, and in the first walking step (the curve colored in blue for the swing leg and the curve colored in magenta for the stance leg), the trajectory converges to the desired period-1 fixed point $x_{*}^{-}$. Thus, in the next step and just after the impact, the trajectory starts with the state $x_{*}^{+}$. The trajectory goes then from the initial point $x_{*}^{+}$to the desired final point $x_{*}^{-}$, and the period of this travel is always fix and equal to $\tau_{*}=0.7647$.

Figure 18(b) reveals the temporal evolution of the applied control law $u$. It is obvious that the compass biped walker requires a considerable energy to be applied on it in order to follow the desired planned trajectory. Compared to the previous control approach based on the passive dynamic walking (see Figure 8(c)), the amplitudes of the two controllers $u_{h}$ and $u_{s}$ are considerably high when the tracking was achieved.

5.2.2. Second Case: $\varphi=8.3^{\circ}$ and $a=0.8$. We consider now the second case using the following parameters: $\varphi=8.3^{\circ}$ and $a=0.8$, for which the chaotic passive gait is revealed by the chaotic attractor given by Figure $7(b)$. For such set of parameters, the desired period-1 gait has the following step period $\tau_{*}=0.5222$. Figure 19 shows the obtained simulation results. Note that we have considered the initial point $x_{0}$ as previously. Obviously, the results in Figure 19(a) reveal the same observation captured in Figure 18(a), where the biped robot needs only one walking step to reach and then follow the reference trajectory. Figure 19(b) shows the control law $u$. We have also obtained almost the same result as in Figure 18(b). The only remarkable difference, which is quite reasonable, lies in the amplitude of the controller $u_{s}$ during the first step. This high level of the amplitude comes from the fact that the selected departure condition $x_{0}$ was taken far away from the reference trajectory. Thus, the biped robot needs more control effort to reach the desired trajectory.

It is worth to note that after these investigations, the use of the second control approach of a designed trajectory needs much control efforts compared to the first control approach based on tracking an entirely passive dynamic walking generated from the dynamic model of the compassgait biped walker. We can say therefore that the first control approach is more efficient and also more attractive in terms of energy consumption. This result shows the great interest in the study and the use of the passive dynamic walking to control the locomotion of biped robots. 

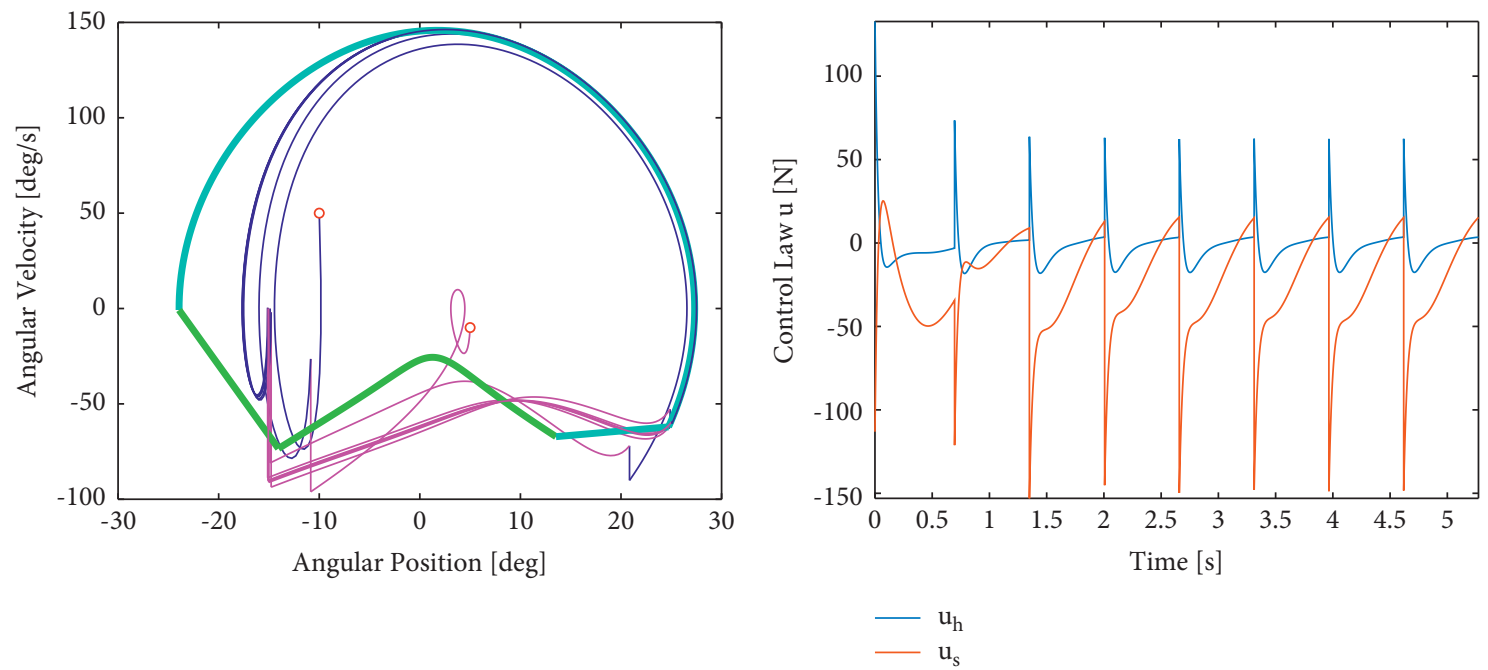

(a)
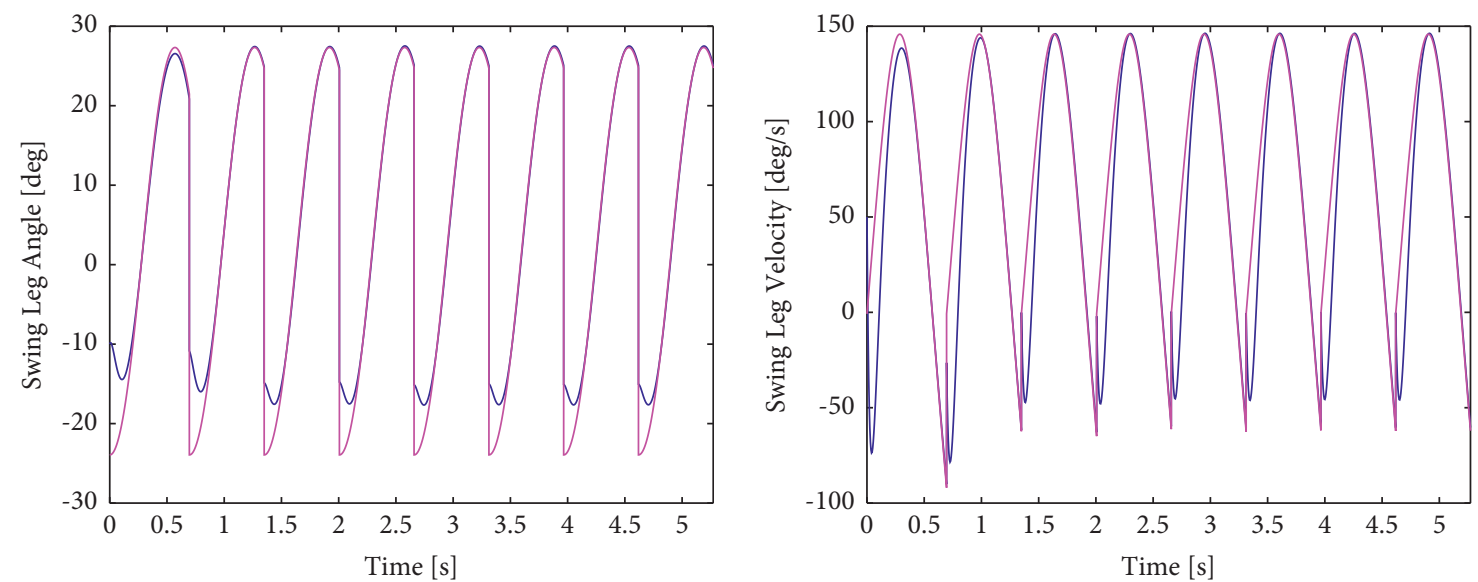

— Controlled Gait

_ Controlled Gait

_ Desired Passive Gait

(c)

(d)

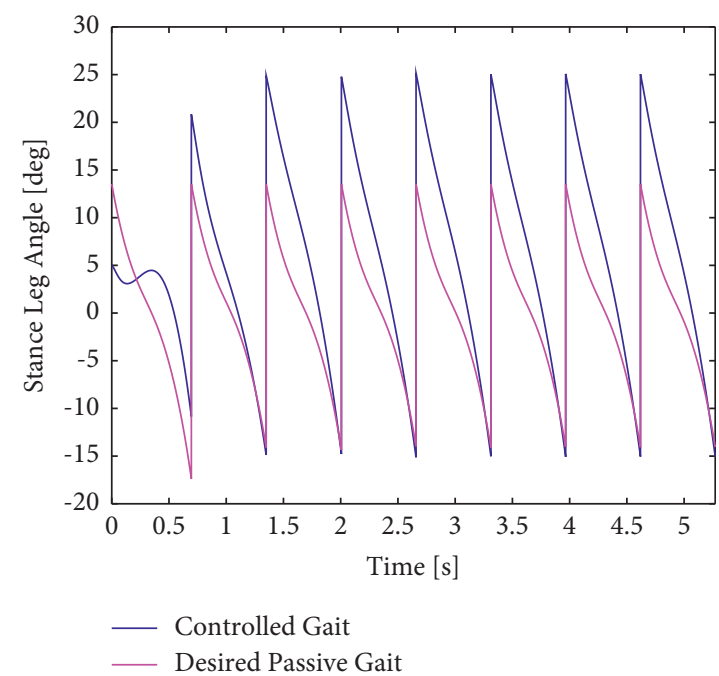

(e)

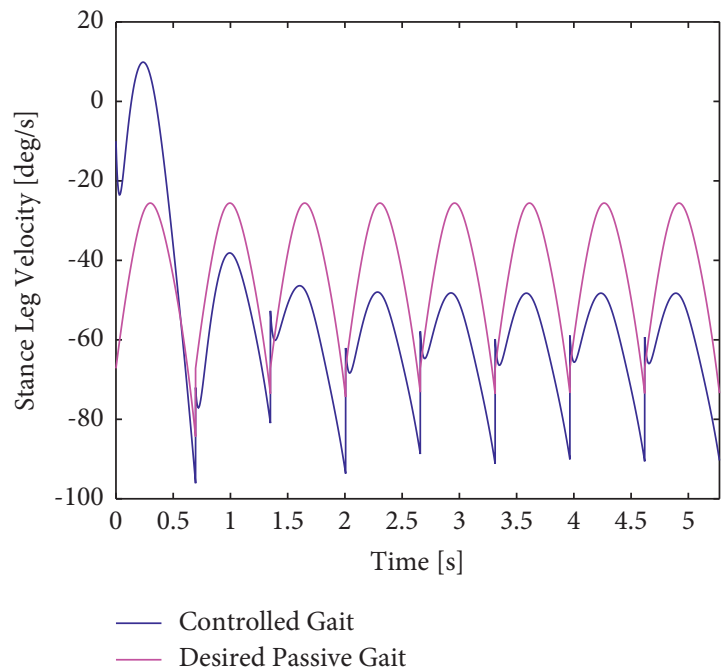

(f)

FIGURE 15: Simulation results revealing the tracking of the desired period-1 passive gait of the compass-gait walker while ascending the slope $\varphi=-5^{\circ}$. The desired period-1 passive gait is obtained for the slope $\varphi=5.2^{\circ}$. Moreover, we fixed $w_{1}=w_{2}=20$. 


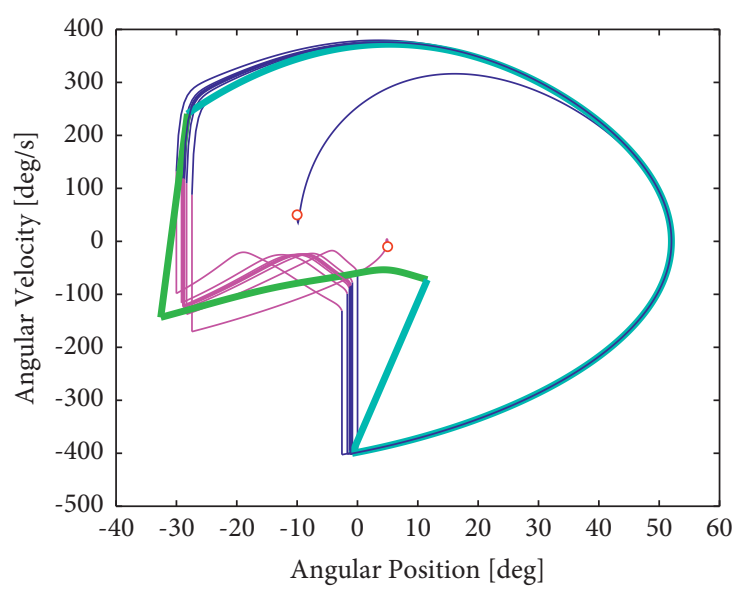

(a)

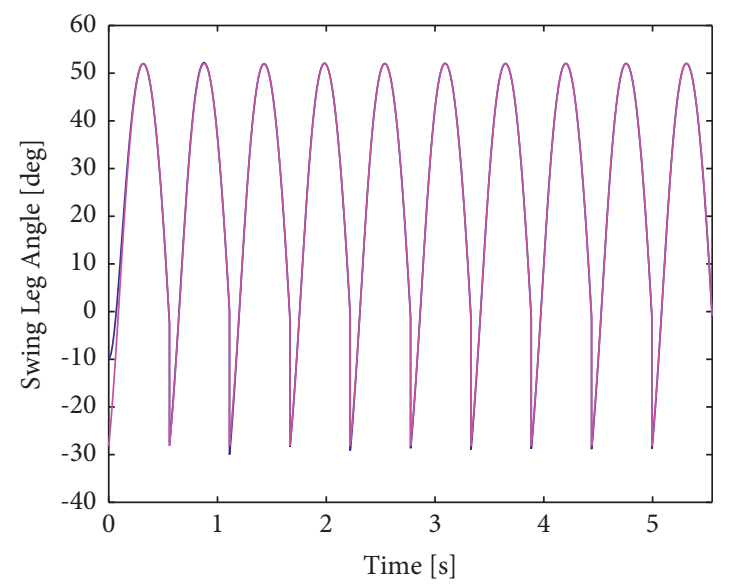

_ Controlled Gait

— Desired Passive Gait

(c)

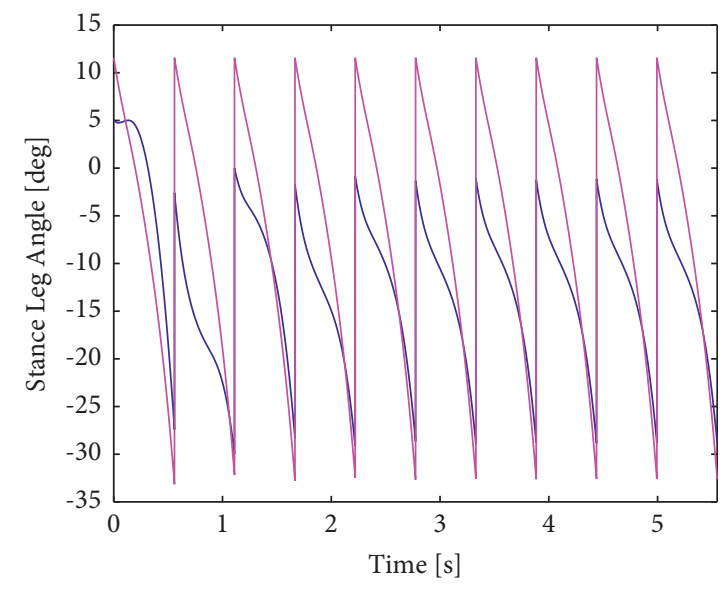

Controlled Gait

Desired Passive Gait

(e)

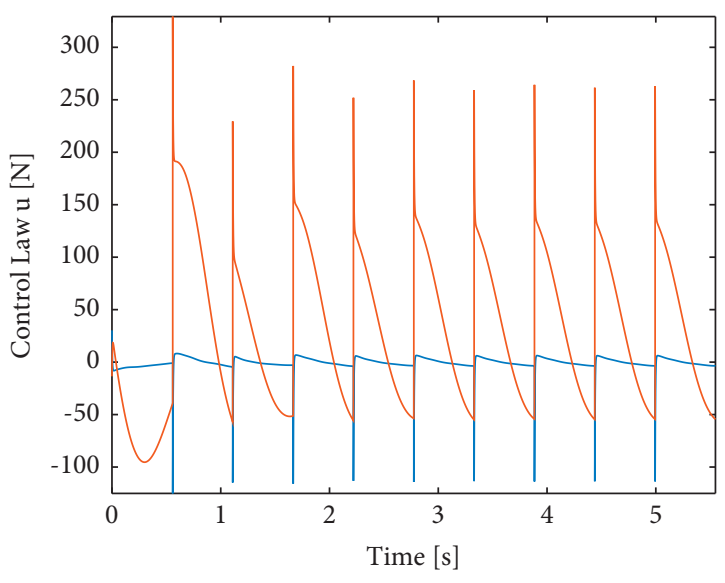

$-\mathrm{u}_{\mathrm{h}}$

(b)

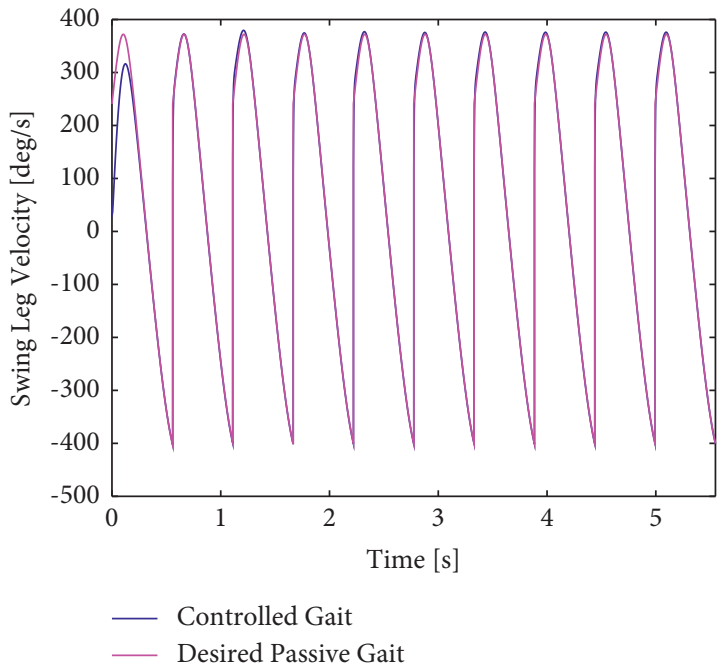

(d)

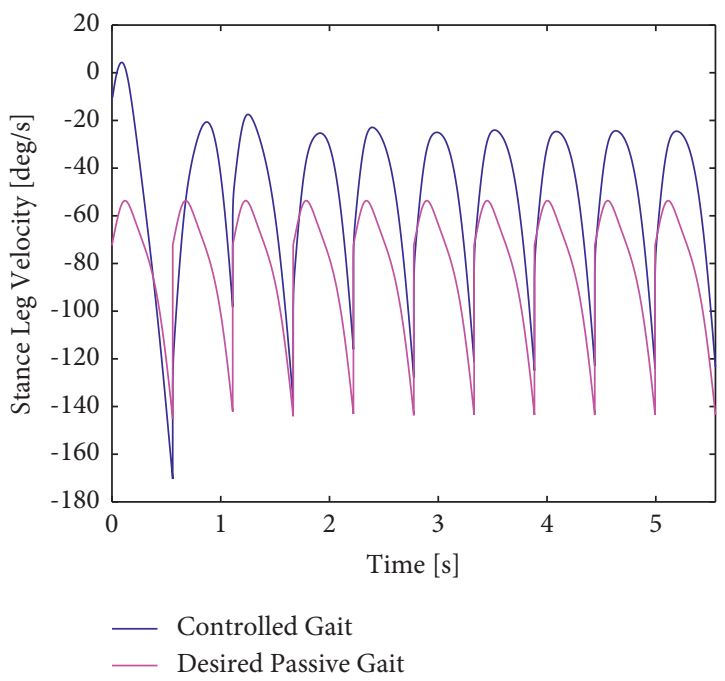

(f)

FIGURE 16: Simulation results showing the tracking of the desired period-1 passive gait of the compass biped robot while walking down the slope $\varphi=15^{\circ}$ and for the leg length's parameter $a=0.8$. The desired period-1 passive gait is obtained for the slope $\varphi=8.3^{\circ}$. 

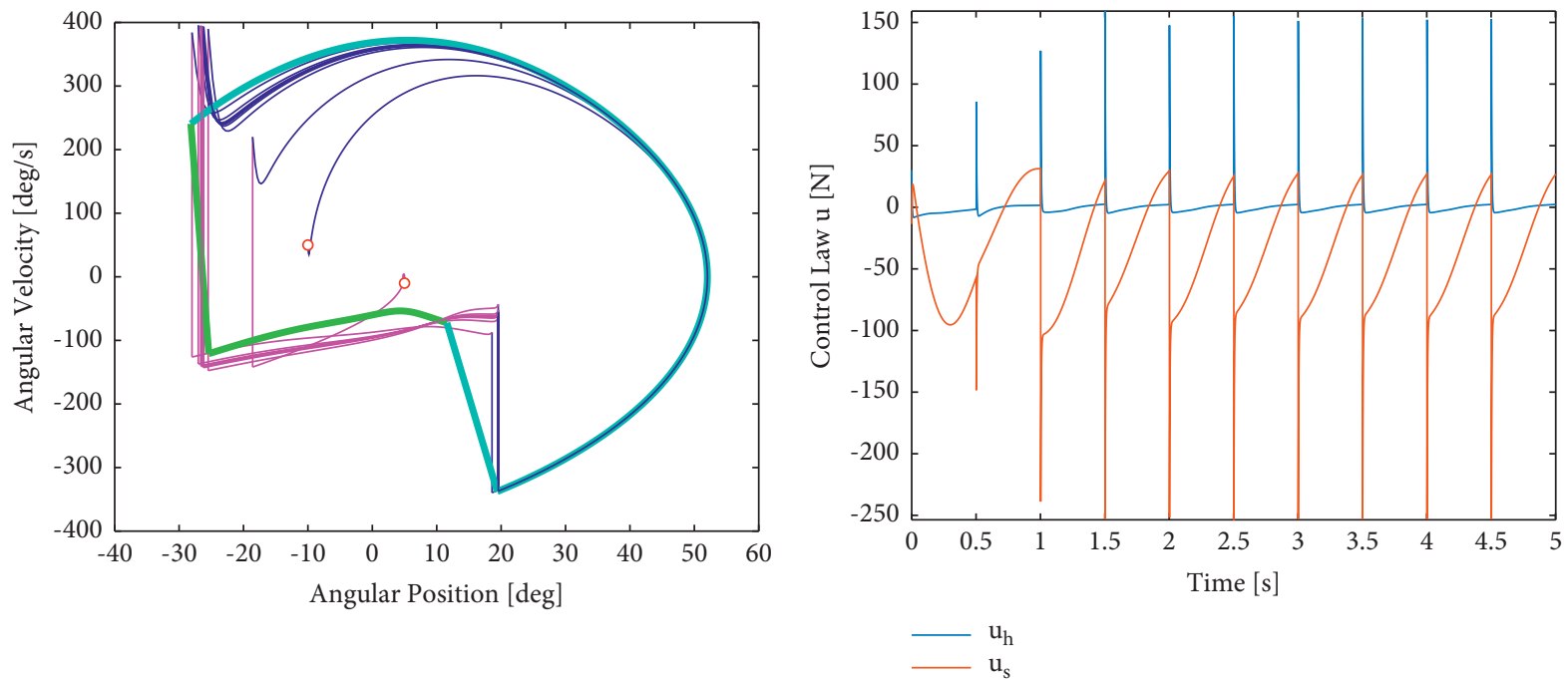

(a)

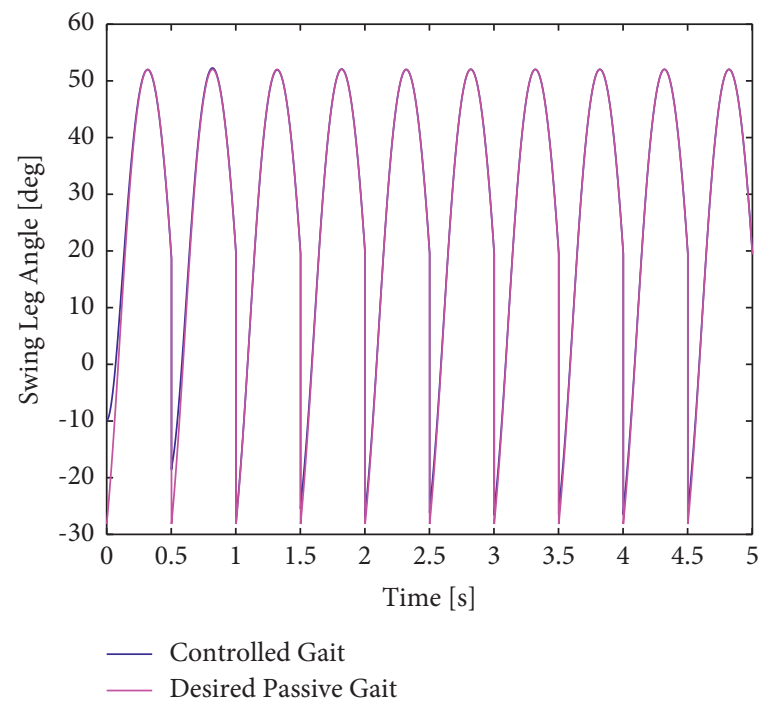

(c)

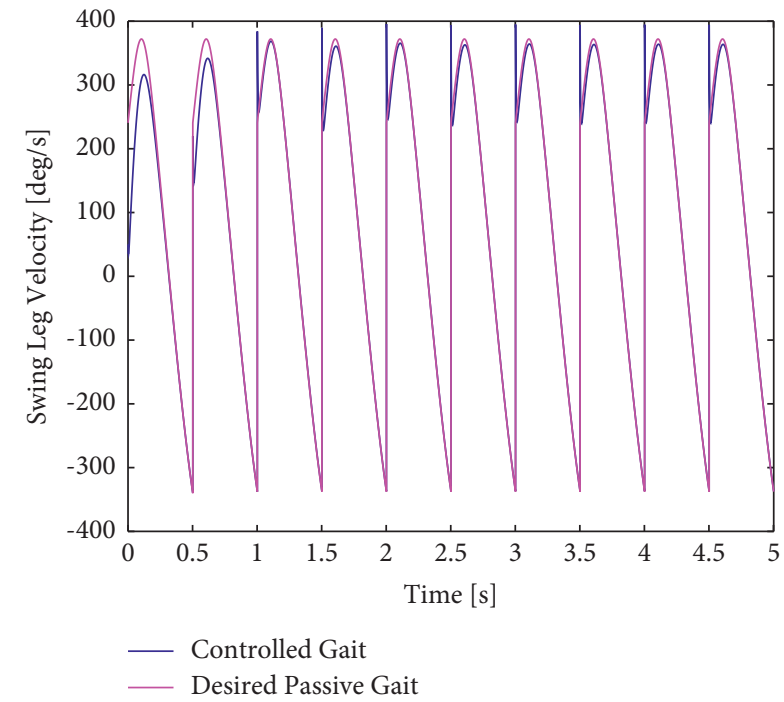

(d)

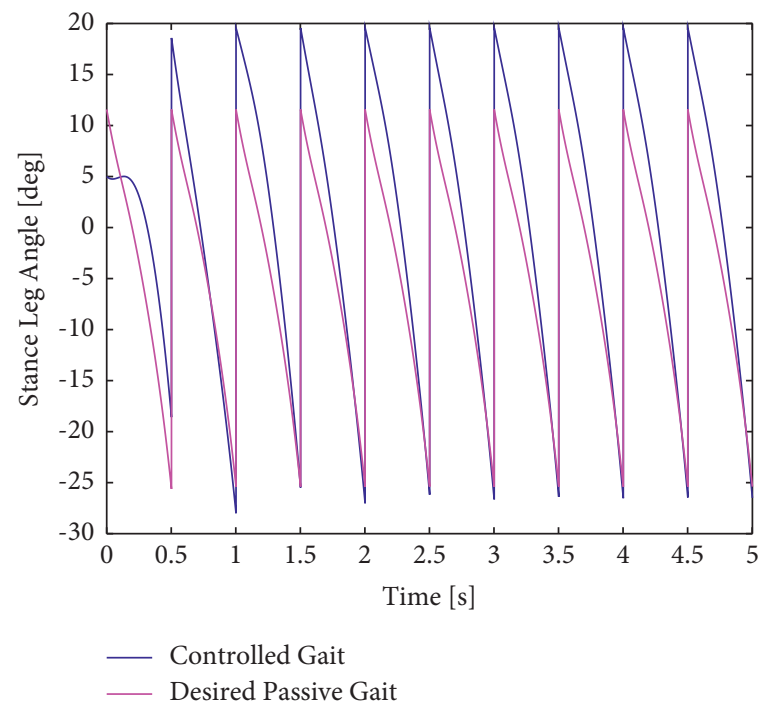

(e)

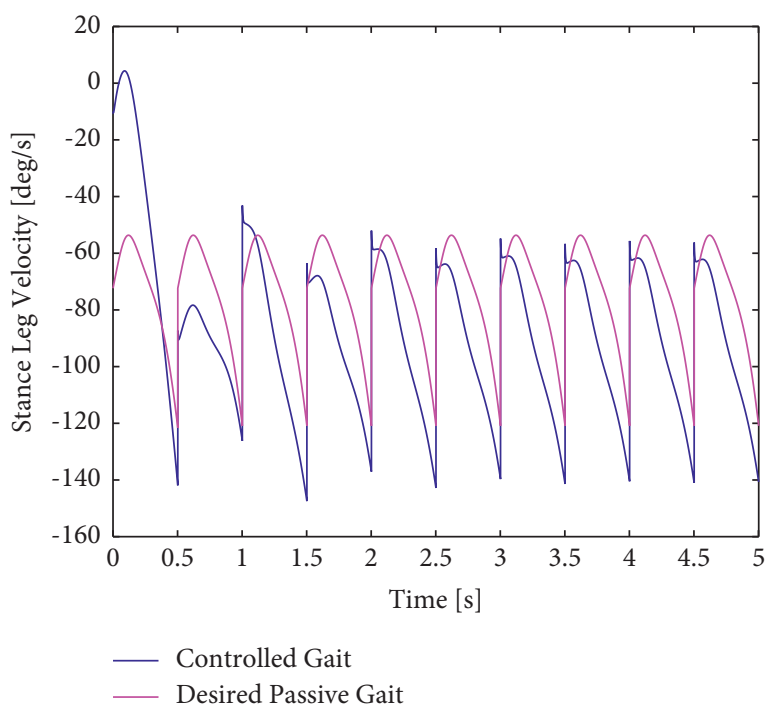

(f)

FIGURE 17: Simulation results revealing the tracking of the desired period-1 passive gait generated for the slope $\varphi=8.3^{\circ}$ and the lower leg segment's length $a=0.8$, while walking on the level ground and then for $\varphi=0^{\circ}$. 
5.2.3. Further Simulation Results for Tracking the 4th-Order Spline Trajectory with $\varphi=5.2^{\circ}$ and $a=0.5$. In this part, we will consider further simulation results showing the effectiveness of adopted control law (23) in the tracking of the designed period-1 order- 4 Spline trajectory. It is worth to note that such desired trajectory depends on the state vector just before impact $x_{*}^{-}$and the nominal step period $\tau_{*}$ obtained for the nominal parameters $\varphi=5.2^{\circ}$ and $a=0.5$. Two different slopes are chosen to verify the efficiency of adopted control law (23) in the tracking of the designed order-4 Spline trajectory, namely, $\varphi=10^{\circ}$ and $\varphi=0^{\circ}$. The initial condition for the simulation of the compass-gait biped walker is adopted to be $x_{0}$ given by (56).

(1) Bipedal Walking for the Slope. $\varphi=10^{\circ}$. Figure 20 shows the simulation results for the slope $\varphi=10^{\circ}$ and the length parameter $a=0.5$. Obviously, in Figure 20(a), the controlled gait of the compass robot converges to the desired gait in only three walking steps. This behavior can be observed in Figure 20(b), where the control effort conserves its oscillation after three steps (notice that a walking step can be understood as the time between two successive transitions in the control law $u$ in Figure 20(b)). As in the first control approach and the previous nominal cases, the control effort $u_{h}$ applied at the hip is smaller than that of the stance leg, $u_{s}$.

(2) Bipedal Walking on the Level Ground. $\varphi=0^{\circ}$. We consider now the case of a bipedal walking on the level ground, and then for $\varphi=0^{\circ}$. The length of the lower leg segment of the two legs is kept to its nominal value, that is, $a=0.5$. Figure 21(a) reveals the controlled attractor and the desired period-1 trajectory. Figure 21(b) depicts the control effort $u$ applied to the biped robot as it walks on the ground. The compass robot walks steadily and with a 1-periodic behavior on the level ground with an acceptable control effort.

\subsubsection{Further Simulation Results for Tracking the 4th-Order} Spline Trajectory with $\varphi=8.3^{\circ}$ and $a=0.8$. Let us consider now the case of the nominal passive gait obtained for the slope $\varphi=8.3^{\circ}$ and the lower leg segment $a=0.8$. As noted previously, the design of the desired 4th-order Spline trajectory depends on the state vector just before impact $x_{*}^{-}$and the step period $\tau_{*}$ obtained for these nominal parameters $\varphi=8.3^{\circ}$ and $a=0.8$. For the bipedal walking of the compass robot, we will select two slopes: $\varphi=15^{\circ}$ and $\varphi=0^{\circ}$. The initial condition for the simulation of the compass-gait biped walker is $x_{0}$ defined by (56).

(1) Bipedal Walking down the Slope. $\varphi=15^{\circ}$. Figure 22 reveals the simulation results of the controlled compass biped robot while walking down the slope $\varphi=15^{\circ}$ and for the lower leg segment length $\varphi=15^{\circ}$. The nominal parameters $x_{*}^{-}$and $\tau_{*}$ for planning the desired period-1 Spline trajectory were computed for the nominal parameters $\varphi=8.3^{\circ}$ and $a=0.8$. Figure 22(b) shows the temporal evolution of the control law $u$. Obviously, and as previously, the controlled gait becomes one-periodic just after three walking steps.
(2) Bipedal Walking on the Level Ground. $\varphi=0^{\circ}$. We consider now the case of bipedal locomotion on the level ground and then for $\varphi=0^{\circ}$. Recall that the nominal parameters adopted for the design of the 4th-order Spline trajectory to be tracked are $\varphi=8.3^{\circ}$ and $a=0.8$. Simulation results are depicted in Figure 23. As previously, the gait of the compass robot is controlled and becomes one-periodic just after three steps.

\section{Comparison}

In this section, we compare the efficiency of the two previous control approaches to control chaos in the compass-gait walker with another approach. The well-known method adopted for the control of chaos in the bipedal walking is the OGY control approach $[26,52]$. Such approach is based on the linearization of the controlled Poincare map $[45,46]$. This OGY control method was adopted in [71] to control chaos in the compass-gait biped robot and in [82] for the torso-driven biped robot. In these works, authors developed a mathematical expression of the constrained (or hybrid) controlled Poincaré map, which has not the classical form, but it was found to be constrained to a transcendental map that should be solved at each walking step. Some simplified versions of this constrained controlled Poincaré map were developed in $[13,40]$ for the compass biped robot and in [83] for the biped robot with torso. In all these previous works, such constrained/hybrid controlled Poincaré map is expressed as follows:

$$
\left\{\begin{array}{l}
x_{k+1}^{-}=\mathscr{P}\left(x_{k}^{-}, u_{k}, \tau_{k}\right), \\
\mathscr{Q}\left(x_{k}^{-}, u_{k}, \tau_{k}\right)=0,
\end{array}\right.
$$

where $x_{k}^{-}$is the state vector just before impact of the swing leg with the ground, $\tau_{k}$ is the step period, and $u_{k}$ is the controller applied during the $k$ th step and remains constant. The first equation in (58) represents the controlled Poincaré map, whereas the second equation defines the constraint that should be solved numerically to find $\tau_{k}$, which will be used next in the Poincare map to iterate it.

In order to design this constrained controlled Poincaré map (58), authors linearized the impulsive hybrid nonlinear system (10a) and (10b) around a desired period-1 passive hybrid limit cycle for some desired slope $\varphi$. Thus, they developed a reduced impulsive hybrid nonlinear/linear system. By means of this simplified system and via some mathematical developments, such expression (58) was therefore obtained.

Recently, authors in $[84,85]$ designed an explicit expression of the Poincaré map, different to (58), and that has the conventional form. They used in [84] the first-order Taylor series expansion, whereas they used in [85] the second-order Taylor approximation. They showed in these works the validity of the developed expression of the Poincaré map in investigating the complex behavior of the passive dynamic walking of the compass-gait bipedal walker. Moreover, they developed in [72] an analytical expression of the controlled Poincaré map to stabilize the passive gaits of the compass robot and then to control chaos. Such 


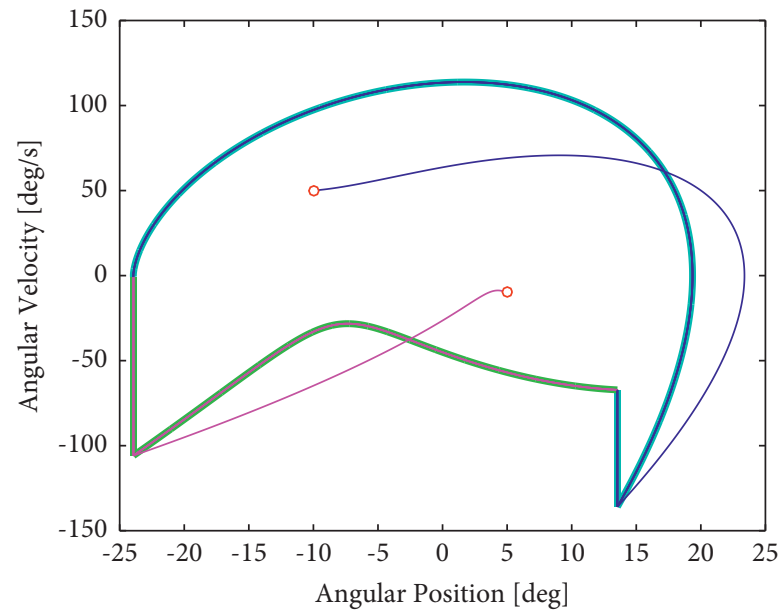

(a)

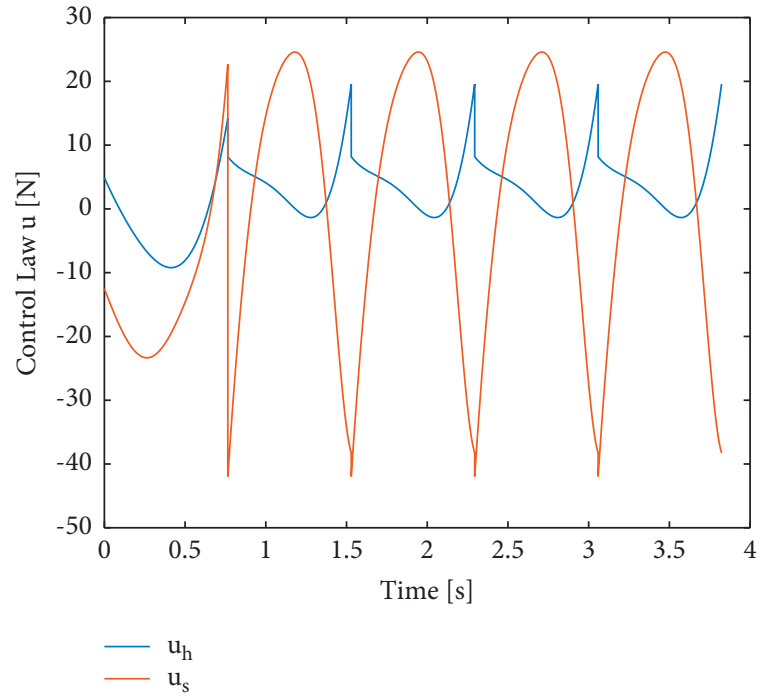

(b)

FIGURE 18: Simulation results showing the controlled chaotic gait of the compass-gait biped walker for $a=0.5$ and $\varphi=5.2^{\circ}$ using the second control approach by designing the 4th-order Spline trajectory.

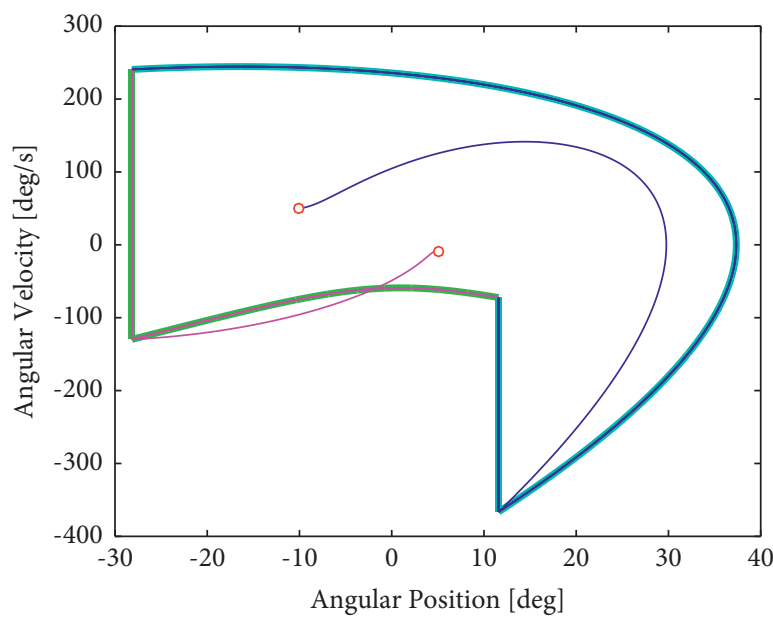

(a)

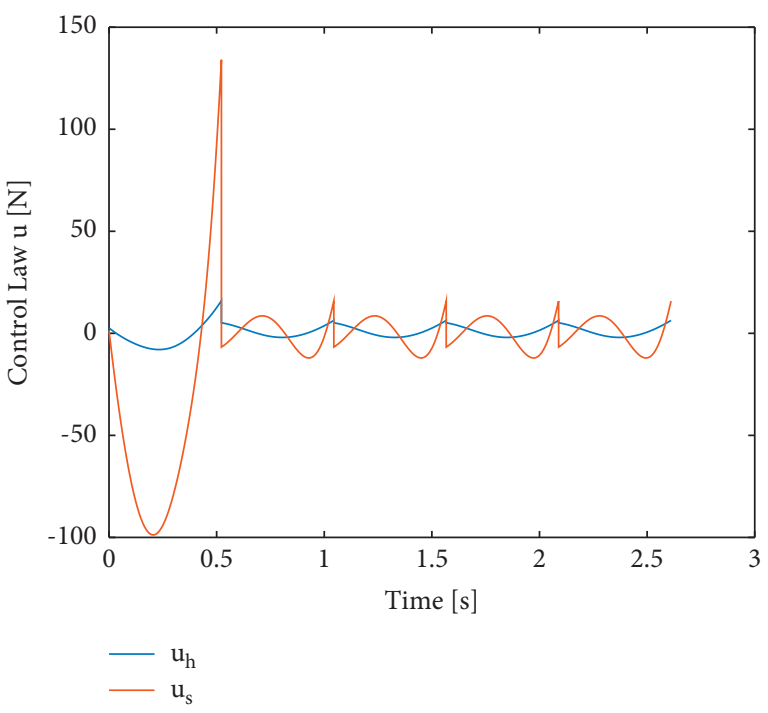

(b)

FIGURE 19: Simulation results of the controlled gait of the compass biped walker for $\varphi=8.3^{\circ}$ and $a=0.8$ and by adopting the second control approach.

controlled Poincaré map has the following expression, as the classical form:

$$
z_{k+1}^{-}=\mathscr{P}\left(z_{k}^{-}, u_{k}\right)
$$

Compared to mapping (58), $z_{k+1}^{-}=\mathscr{P}\left(z_{k}^{-}, u_{k}\right)$. in (59) is a reduced state vector and is defined with respect to the state vector $x_{k}^{-}$like so $z_{k}^{-}=\left[\begin{array}{ll}\mathscr{O}_{3 \times 1} & \mathscr{I}_{3}\end{array}\right] x_{k}^{-}$, where, and in the sequel, $\mathscr{O}$ is the zero matrix and $\mathscr{I}$ is the identity matrix.

For the stabilization of the period-1 fixed point, $z_{*}^{-}$, of uncontrolled Poincaré map (58) or (59), the following state feedback control law was adopted:

$$
u_{k}=K\left(z_{k}^{-}-z_{*}^{-}\right) \text {, }
$$

where $K$ is the feedback control gain designed using the LMI approach [85].

Note that for constrained controlled Poincaré map (58), the control law $u_{k}$ in (60) is defined with respect to the state vectors $x_{k}^{-}$and $x_{*}^{-}$, and the control gain $K$ is of dimension $(1 \times 4)$. In contrast, for reduced Poincaré map (59), the controller gain $K$ is of dimension $(1 \times 3)$. The vector $z_{*}^{-}$(or $x_{*}^{-}$) stands for the state of the period-1 passive gait (or the period-1 passive hybrid limit cycle) just before the impact phase. 


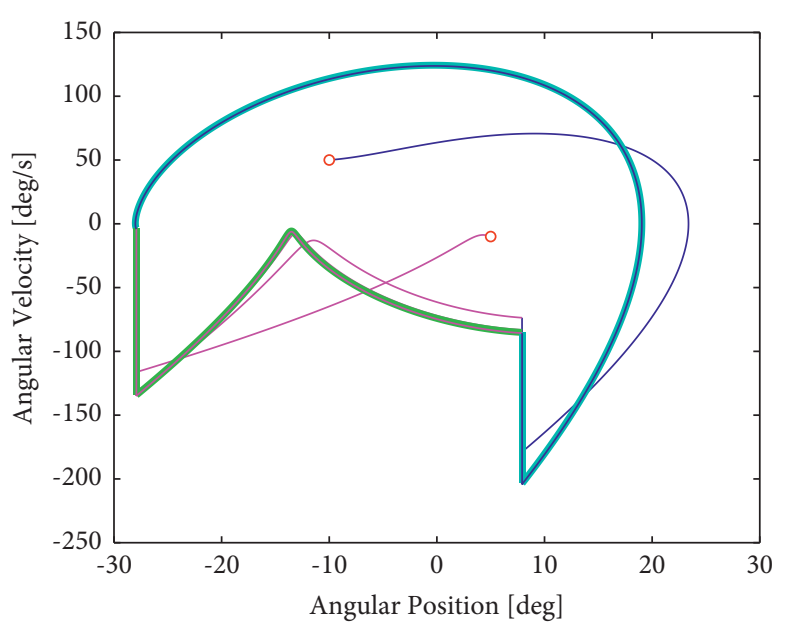

(a)

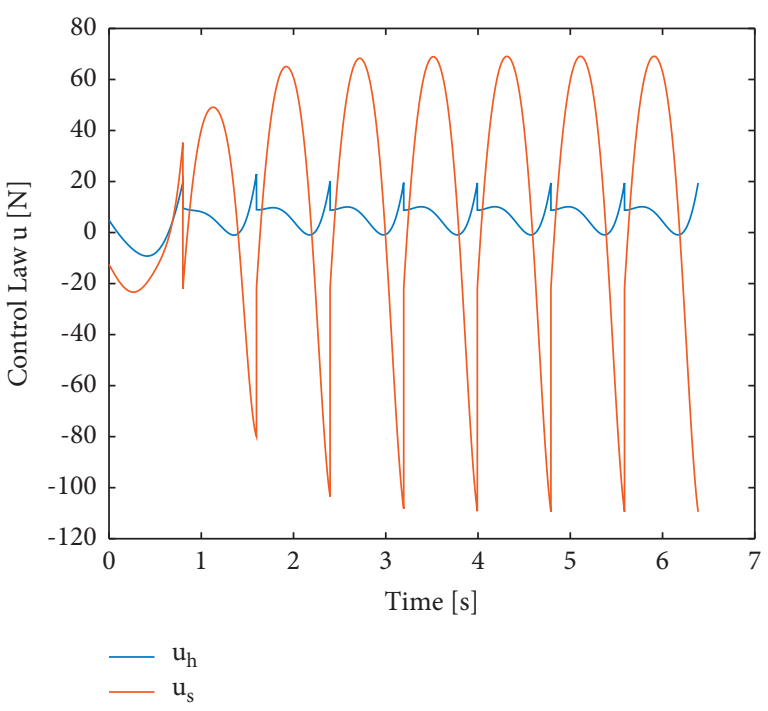

(b)

FiguRE 20: Simulation results of the controlled gait of the compass biped walker using the order-4 Spline trajectory for $\varphi=10^{\circ}$ and $a=0.5$. The nominal parameters for the design of the desired trajectory are $\varphi=5.2^{\circ}$ and $a=0.5$.

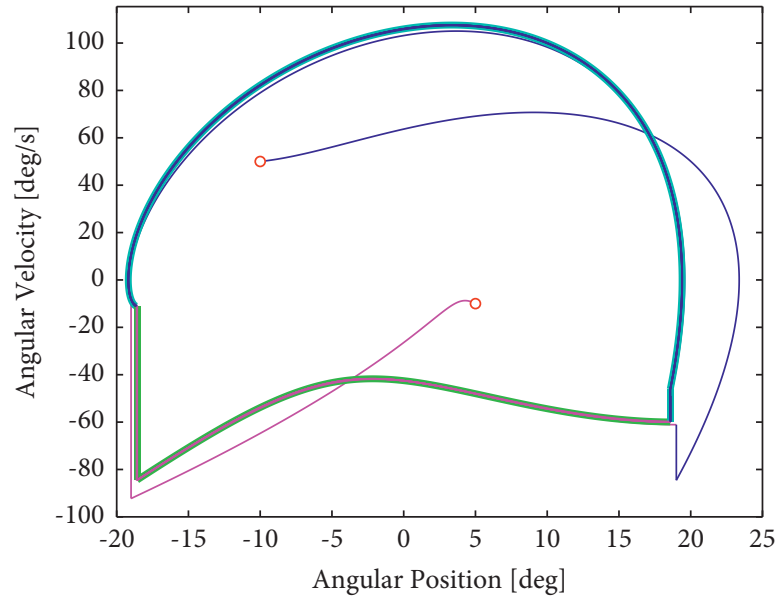

(a)

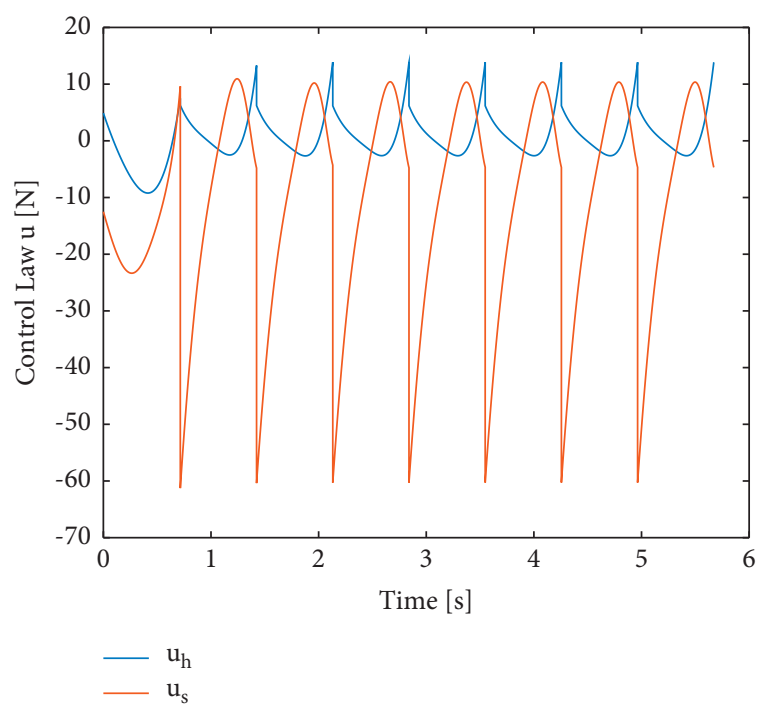

(b)

FIGURE 21: Simulation results of the controlled motion of the compass-gait biped robot using the order-4 Spline trajectory and while walking on the level ground, which is for $\varphi=0^{\circ}$, and with $a=0.5$. The nominal parameters for the design of the desired trajectory are $\varphi=5.2^{\circ}$ and $a=0.5$.

It is worth to note that such control law (60), when applied to impulsive hybrid nonlinear dynamics (10a) and (10b) of the compass walker, remains constant during a walking step. It only updates its value at the impact. In addition, an important remark revealed from the previous works $[71,82,85]$ using controlled Poincaré map (58) or (59), and then state feedback control law (60), is that the control amplitude used for the control of chaos and the stabilization of the passive bipedal walking is very low. This fact reveals then the energy efficiency of such control law (60) for bipedal robots.
In the sequel, we will use this OGY-based state feedback control law (60) for comparison. We note that in the works realized in [71, 85] for the compass-gait walker, only one control input at the hip was considered. Thus, as in the present work we have two control inputs, $u_{h}$ and $u_{s}$, then we will extend the work realized in [85] for the design of controlled Poincare map (58) and then for the computation of the feedback control gain $K$ by considering these two control inputs. Accordingly, the dimension of $K$ becomes $(2 \times 3)$. 


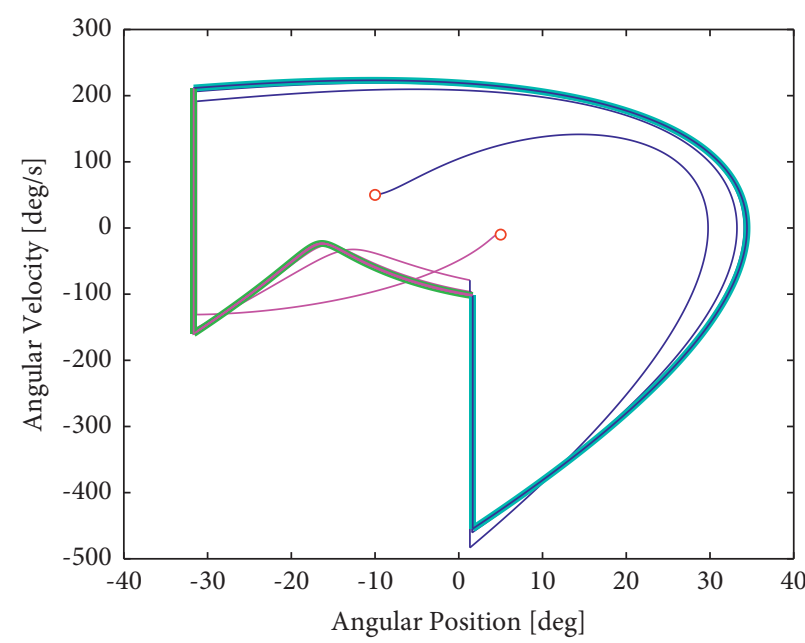

(a)

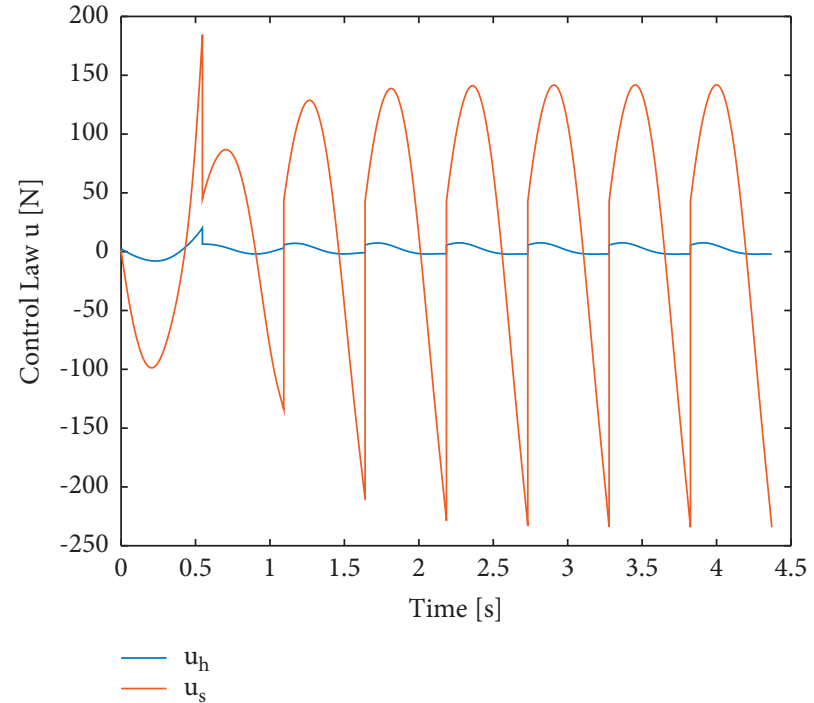

(b)

FIGURE 22: Simulation results of the controlled compass-gait biped robot descending the inclined surface of slope $\varphi=15^{\circ}$ and for $a=0.5$. The nominal parameters for the trajectory design are $\varphi=8.3^{\circ}$ and $a=0.8$.

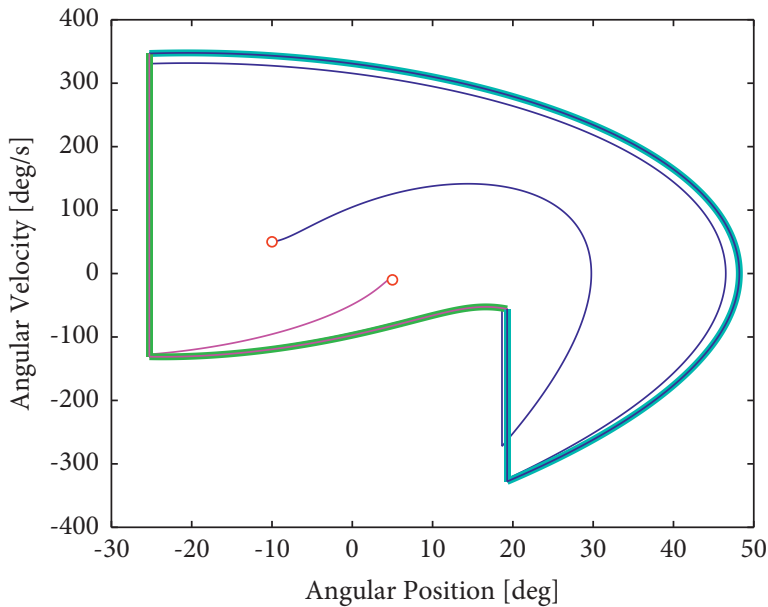

(a)

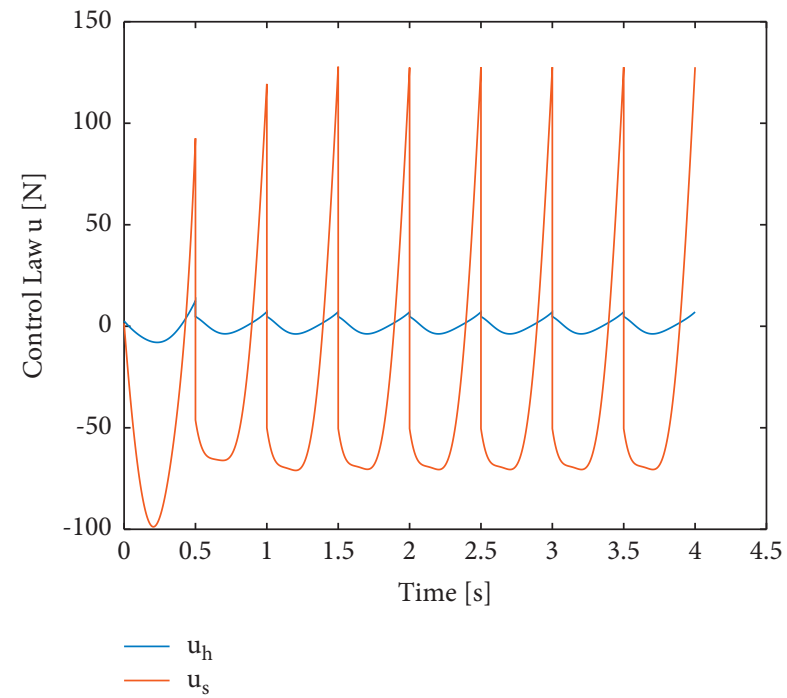

(b)

FIGURE 23: Simulation results of the controlled gait of the compass robot while walking on the level ground, $\varphi=0^{\circ}$, by adopting the second control approach using the order- 4 Spline trajectory and by choosing $a=0.8$. The nominal parameters for the design of the desired trajectory are $\varphi=8.3^{\circ}$ and $a=0.8$.

Note that in the previous simulations in Section 4, we used the state vector $x$, and then the initial condition for simulation was $x_{0}$. Thus, as OGY-based state feedback control law (60) depends on the reduced state vector just before impact $z_{k}^{-}$, then and for the simulation purpose, the initial condition $z_{0}^{-}$used for the computation of the initial vector of control law (60) to be applied to the compass robot at the first walking step can be computed from the state vector just after impact $z_{0}^{+}$using mainly the algebraic equation in (10b) and condition (7), as follows:

$$
\begin{aligned}
& z_{0}^{-}=\left[\begin{array}{ll}
\mathcal{O}_{3 \times 1} & \mathscr{I}_{3}
\end{array}\right] x_{0}^{-}, \\
& x_{0}^{-}=\left[\begin{array}{cc}
R & \mathcal{O}_{2 \times 2} \\
\mathcal{O}_{2 \times 2} & Q_{m}^{-1} Q_{p}
\end{array}\right] x_{0}^{+}, \\
& x_{0}^{+}=\left[\begin{array}{c}
-\widehat{C} \\
\mathscr{I}_{3}
\end{array}\right] z_{0}^{-}+\left[\begin{array}{c}
-2 \varphi \\
\mathcal{O}_{3 \times 1}
\end{array}\right],
\end{aligned}
$$

where $\widehat{C}=\left[\begin{array}{lll}1 & 0 & 0\end{array}\right]$.

Moreover, in order to apply the OGY-based state feedback control law, the initial condition $x_{0}^{+}$should belong 


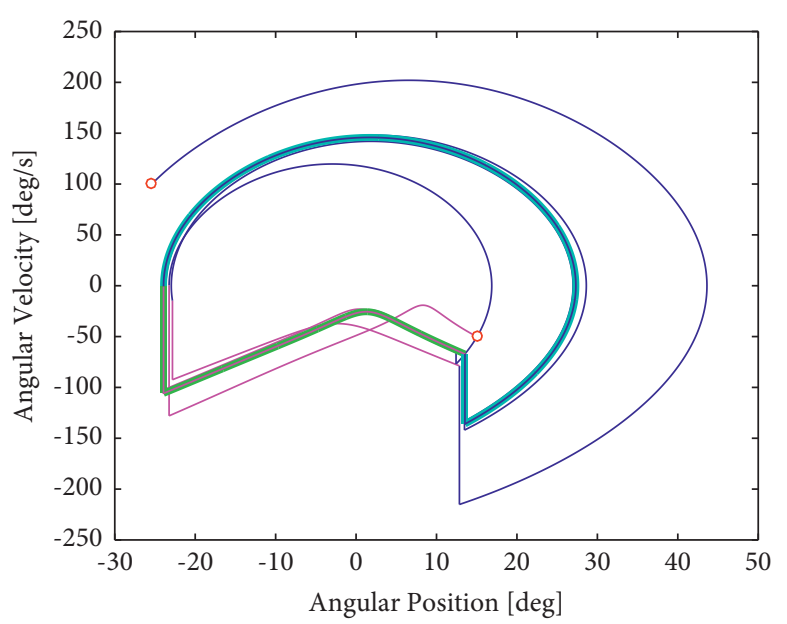

(a)

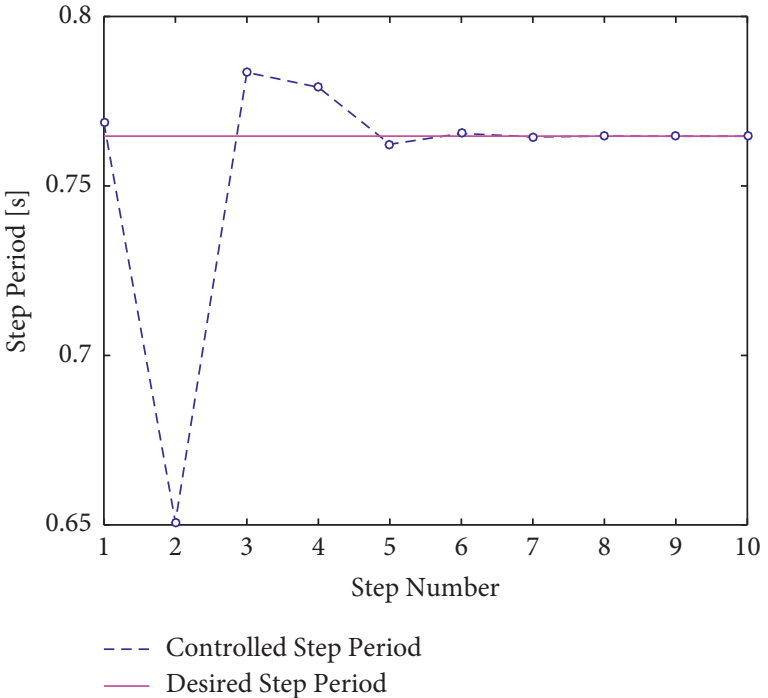

(b)

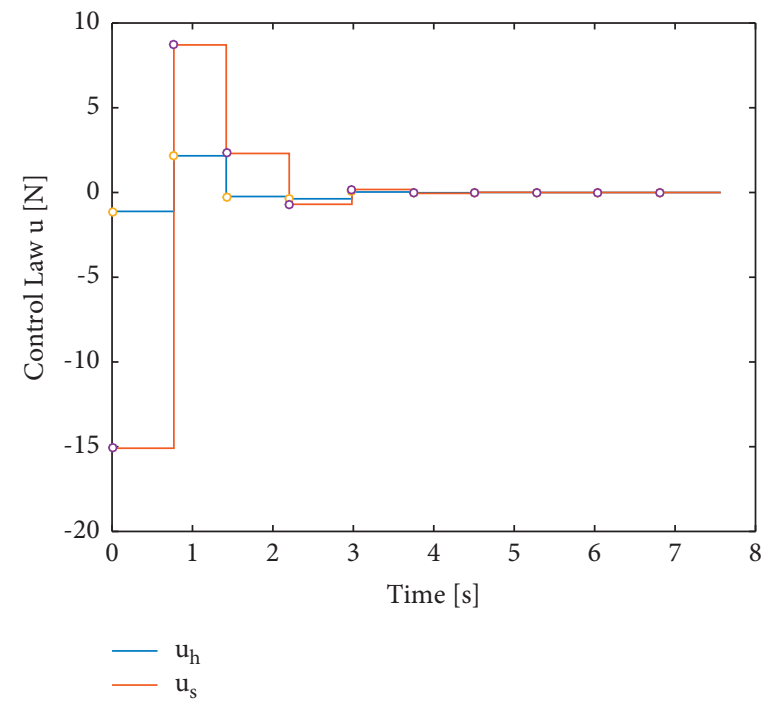

(c)

Figure 24: Simulation results of the controlled chaotic passive gait of the compass-gait walker for the parameters $a=0.5$ and $\varphi=5.2^{\circ}$ using the OGY-based state feedback control law $u_{k}$ in (60) and the initial condition $x_{0}^{+}$in (66).

to the impact set $\Gamma$ defined by (11). Then, we should select $x_{0}^{+}$ such that

$$
C x_{0}^{+}+2 \varphi=0 .
$$

Therefore, compared to the simulations realized in the previous section, the choice of the initial condition $x_{0}^{+}$ cannot be arbitrary, as we have done previously. It should imperatively satisfy condition (64). Thus, in order to make a comparison, we will realize our two previous control approaches adopted in the present work using a different initial condition that should be used then for the OGY-based control approach. Moreover, we will adopt the same set of parameters $\varphi$ and $a$ used in the previous section.
6.1. Simulation Results for $a=0.5$ and $\varphi=5.2^{\circ}$. We consider here the first scenario of the bipedal walking for which $a=$ 0.5 and $\varphi=5.2^{\circ}$. For such set of parameters, the period- 1 fixed point just after impact, $x_{*}^{+}$, associated to the desired period-1 hybrid limit cycle embedded inside the chaotic attractor is given by (55). Using relations (61) and (62), it is possible to calculate the reduced state vector just before impact $z_{*}^{-}$to be used for OGY-based state feedback control law (60). Moreover, using the approach introduced in [72] for the computation of the feedback gain $K$ of controller (60) via the design of the explicit expression of controlled Poincare mapping (60), the controller gain $K$ is obtained as follows: 


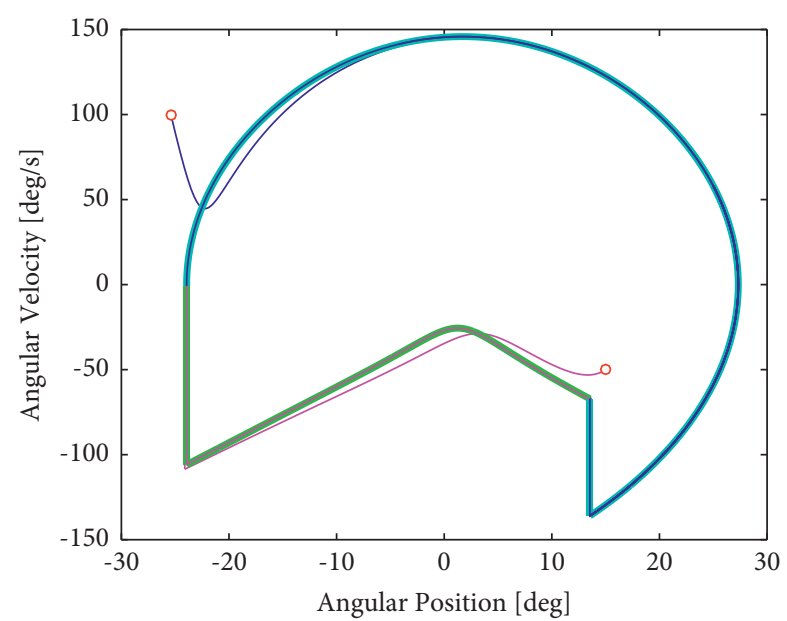

(a)

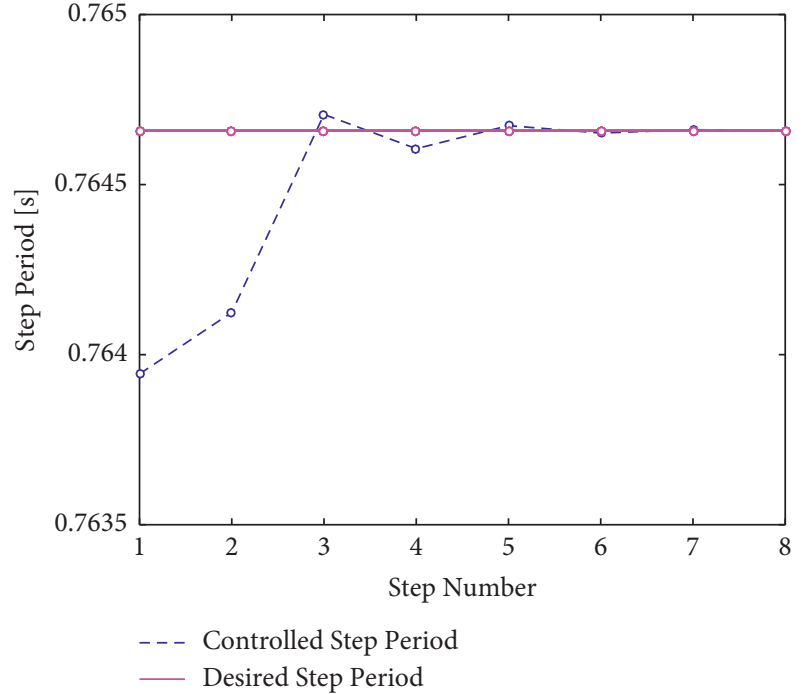

(b)

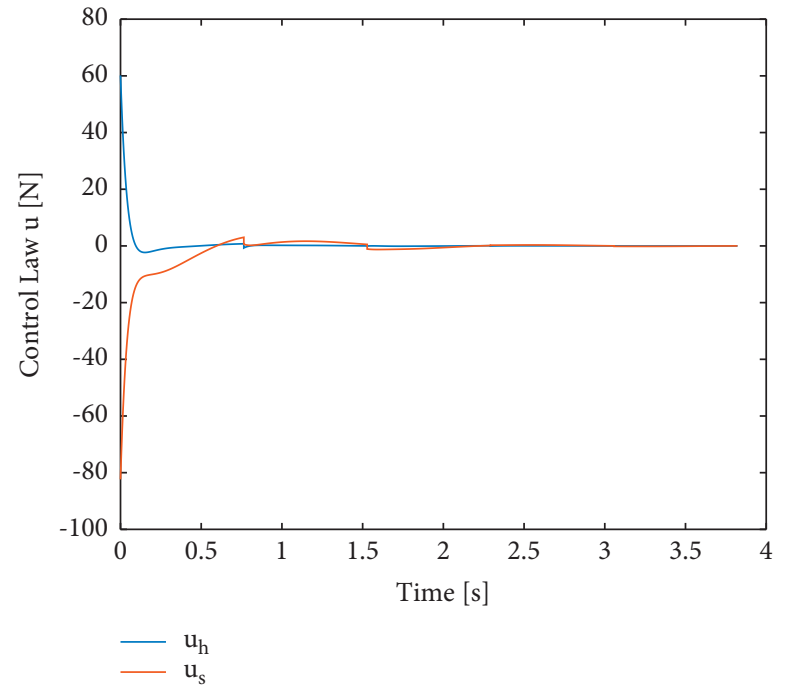

(c)

FigURE 25: Simulation results of the controlled bipedal gait for the set of parameters $a=0.5$ and $\varphi=5.2^{\circ}$, using the first control approach based on the passive dynamic walking. The same initial condition $x_{0}^{+}$in (66) as in Figure 24 is used here.

$$
K=\left[\begin{array}{ccc}
35.0024 & 0.7880 & -7.3465 \\
260.1874 & 3.5781 & -27.2967
\end{array}\right] \text {. }
$$

Using then this gain $K$ and the period-1 fixed point $z_{*}^{-}$in control law (60) and then to impulsive hybrid nonlinear dynamics (10a) and (10b) of the compass-gait walker, the bipedal waking will be stabilized and chaos will be therefore controlled. To show this fact and also for comparison, we consider first the following initial condition:

$$
x_{0}^{+}=\left[\begin{array}{llll}
-25.4 & 15 & 100 & -50
\end{array}\right] .
$$

Recall that the first initial condition $z_{0}^{-}$for control law (60) is calculated via relations (61) and (62). Moreover, the first element, -25.4 , in the state vector $x_{0}^{+}$in (66) is calculated according to expression (64). Thus, such initial condition $x_{0}^{+}$ will be used for the OGY-based control method and also for the two adopted control methods proposed in this work.

The simulation results revealing the behavior of the bipedal walking of the compass robot under OGY-based state feedback control law (60) are given in Figure 24. The simulation results obtained using the first control approach based on tracking the passive dynamic walking of the biped robot itself are given in Figure 25, whereas those obtained via the second control approach based on the 4th-order Spline trajectory are shown in Figure 26. All these results reveal that the chaotic passive gait is well controlled and hence the behavior becomes one-periodic, as shown in Figures 24(a), 25(a), and 26(a). In Figure 24(b), the chaotic gait needs almost 7 steps to be stabilized, where the step period of the controlled gait converges to the desired step period. The same observation can be revealed from 25(b). In 26(b), and 


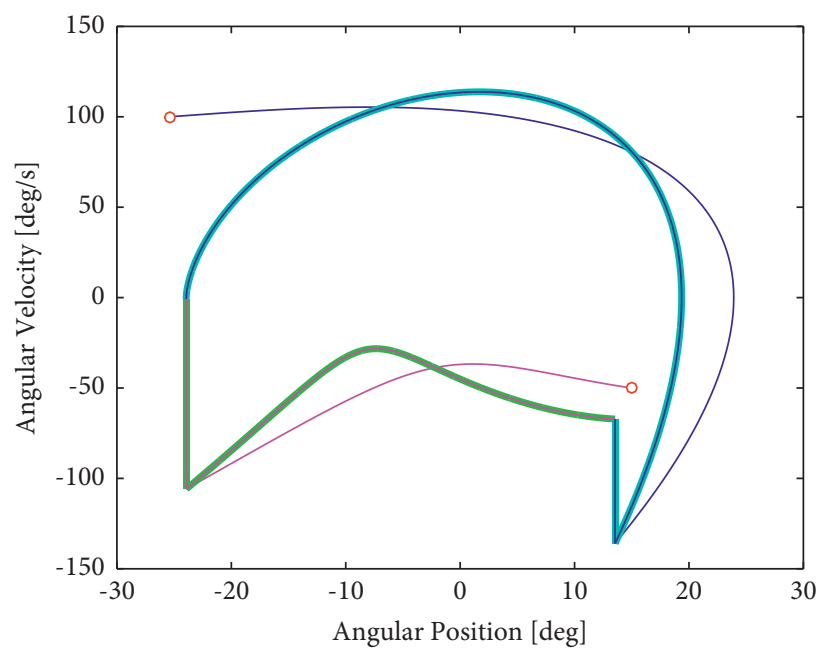

(a)

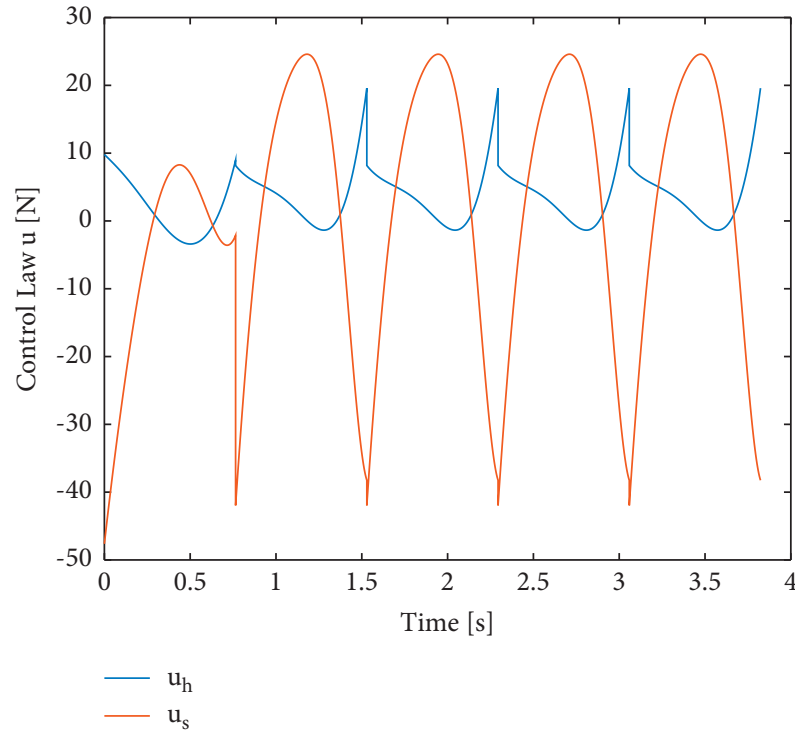

(b)

FiguRE 26: Simulation results showing the controlled chaotic gait for the parameters $a=0.5$ and $\varphi=5.2^{\circ}$ and using the 4 th-order Spline trajectory.

as reported in the previous section, the passive gait is controlled into a single walking step.

The clear difference between the three control approaches lies in the control effort applied to the compass-gait walker. Indeed, in the OGY-based control approach, and then in Figure 24(c), the maximum amplitude of the control law, and more particularly of the control input of the stance leg $u_{s}$, is about $-15[N]$. This value was applied at the first walking step and remains constant during this first step. At the first step, the new control effort applied to the stance leg is around $9[N]$. The value of control law $u_{s}$ decreases progressively after each step and converges to zero. In contrast, the control effort applied at the hip $u_{h}$ is relatively small and converges to zero after almost 5 seconds. Using now the first control approach adopted in this work, and then relying on Figure 25(c), the two control inputs $u_{s}$ and $u_{h}$ converge to zero after almost 3 seconds. Nevertheless, the amplitude of these controllers is relatively high at the first step. The maximum value of the amplitude of the signal $u_{h}$ is around $60[N]$ and that of the signal $u_{s}$ is about $-80[N]$. The control effort of the two controllers decreases and converges to zero. However, compared to the OGY-based control method, the convergence of the controlled chaotic gait to the desired period-1 passive gait using the first control approach is faster as it is clearly depicted in Figures 24(a) and 25(a). This shows the superiority of the control method using the tracking based on the passive dynamic walking of the compass walker itself. For the second control approach adopted in this work, and then for the results in Figure 26, there is no clear difference with the results reported in Figure 18 . The only difference lies in the control signals applied at the first walking step. The other steps are identical since the passive chaotic gait is controlled into only one step and also as the initial condition $x_{0}^{+}$is modified. As noted in the previous section, the control of the passive gait by tracking the planned 4th-order Spline trajectory needs an important control effort during each step and this behavior remains as is while the biped robot walks.

As the OGY-based control method and the first control approach are both entirely based on the passive dynamic walking of the compass biped robot, then and as revealed by the simulation results, the controlled chaotic gait becomes one-periodic and also becomes (almost) passive. In fact, a very weak control effort $u$ is required to remain the gait at the desired one. Therefore, we can conclude that the controlled gait becomes nearly passive. Nevertheless, as discussed previously, the tracking-based control method is faster than the OGY-based control approach.

Actually, we have modified the previous initial condition $x_{0}^{+}$given by (66), and we have adopted that selected in the previous section using the two proposed tracking-based control approaches, that is, state (56), but with a minor rectification in order to satisfy constraint (64). Such new initial condition is

$$
x_{0}^{+}=\left[\begin{array}{llll}
-15.4 & 5 & 50 & -10
\end{array}\right] \text {. }
$$

However, starting from this initial state $x_{0}^{+}$and by applying the OGY-based control method, and then control law (60) with matrix gain $K(65)$, the compass-gait biped walker falls down from the first step. This result reveals the superiority of the two control approaches proposed in this work compared with the OGY-based control method.

6.2. Simulation Results for $a=0.8$ and $\varphi=8.3^{\circ}$. Recall that for the parameters $a=0.8$ and $\varphi=8.3^{\circ}$, the chaotic attractor of the passive bipedal gait of the compass walker 


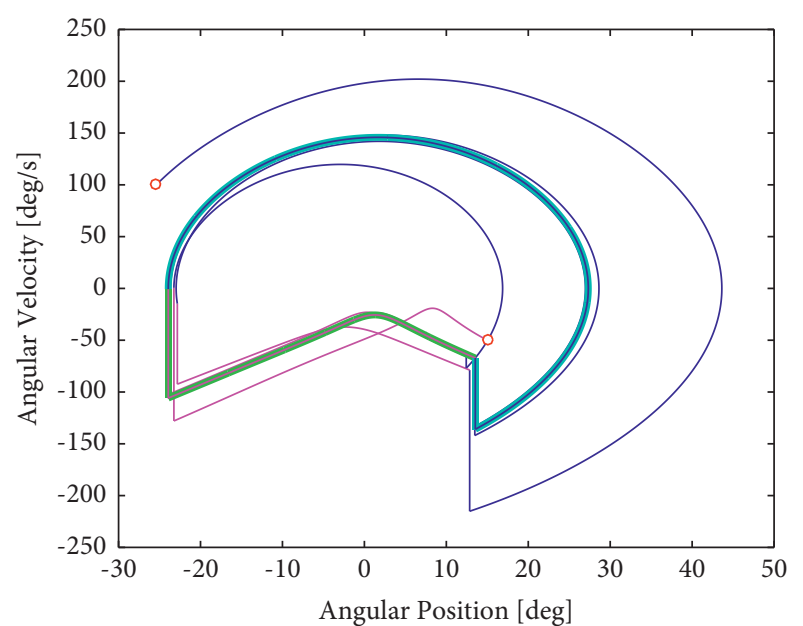

(a)

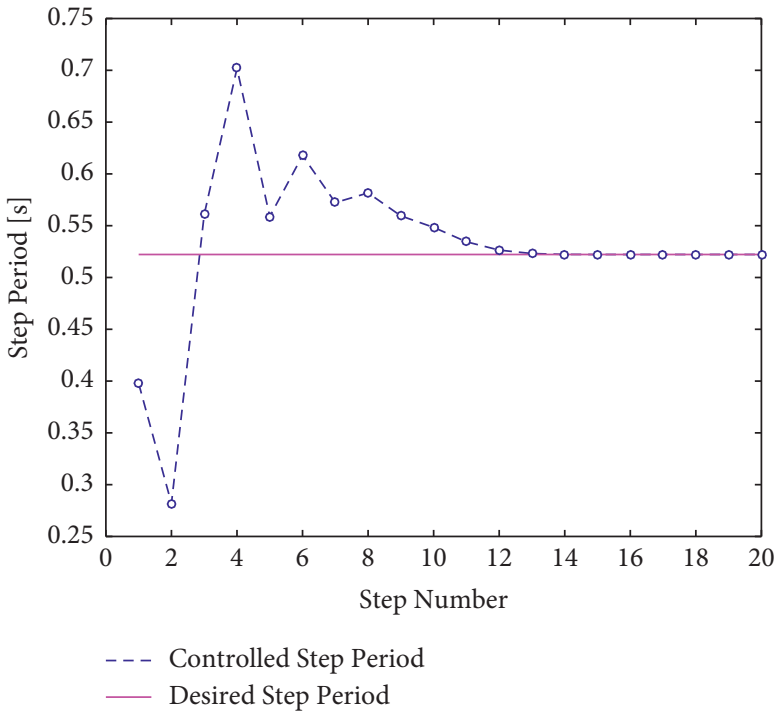

(b)

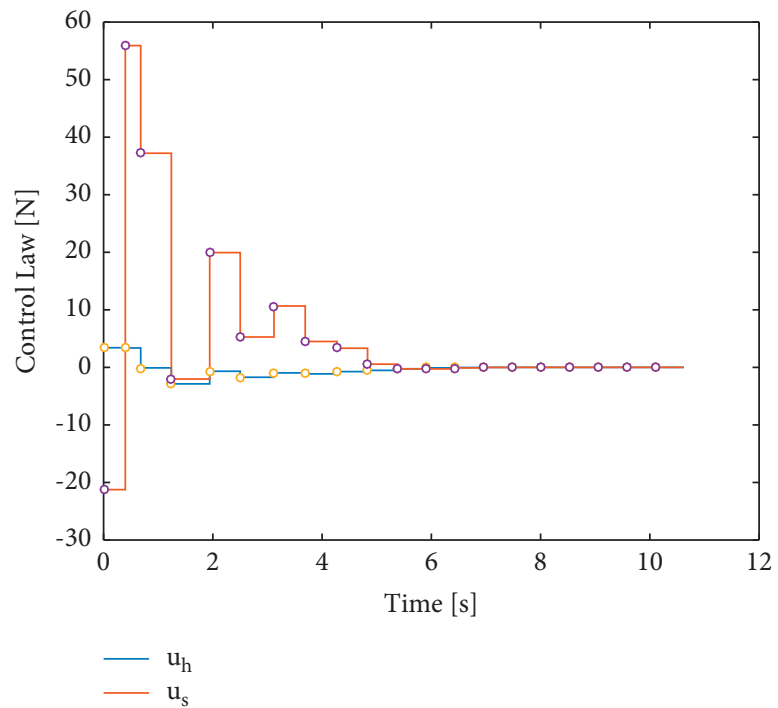

(c)

FIGURE 27: Simulation results showing the controlled gait of the compass-gait bipedal robot for $a=0.8$ and $\varphi=8.3^{\circ}$ by applying the OGYbased state feedback control law $u_{k}$ in (60) and the initial condition $x_{0}^{+}$in (69).

is given by Figure 7(b). Moreover, the period-1 fixed point just after impact, $x_{*}^{+}$, of the desired period-1 hybrid limit cycle is given by (57) and its associated step period is $\tau_{*}=0.5222$. As noted previously, the reduced state vector just before impact $z_{*}^{-}$that will be used in OGY-based state feedback control law (60) is computed relying on relations (61) and (62). Moreover, based on [72], the matrix gain $K$ of this OGY-based control law (60) is computed to be as follows:

$$
K=\left[\begin{array}{ccc}
14.6513 & 0.2120 & -4.4529 \\
381.7472 & 1.6166 & -17.6538
\end{array}\right]
$$

The initial condition $x_{0}^{+}$adopted for the simulation in this second scenario is

$$
x_{0}^{+}=\left[\begin{array}{llll}
-36.6 & 20 & 350 & -100
\end{array}\right] \text {. }
$$

As in the previous case for initial condition (66), the first element, -36.6 , in the previous state vector $x_{0}^{+}$is calculated according to expression (64).

Using then matrix gain $K(68)$ in OGY-based control law (60) and by applying it to the impulsive hybrid nonlinear dynamics of the dynamic walking of the compass-gait walker, we obtain the simulation results in Figure 27. It is obvious that the chaotic gait is controlled and the behavior becomes oneperiodic and also becomes the desired one. Emanating from the initial state $x_{0}^{+}$given by (69), and according to Figure 27(a), the bipedal gait experiences first some long and short walking steps, before it converges to the desired period-1 gait after about 15 steps, as seen in Figure 27(b). From Figure 27(c), the control 


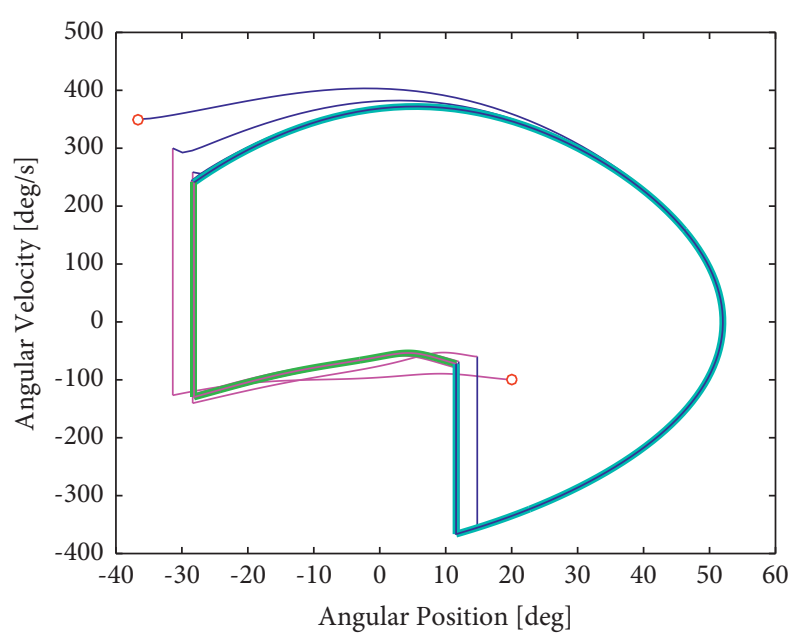

(a)

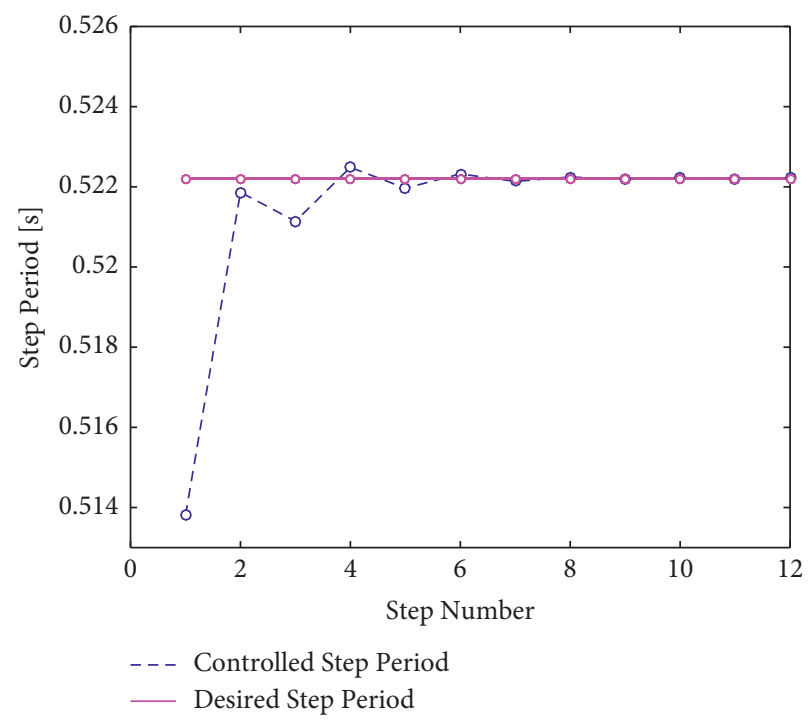

(b)

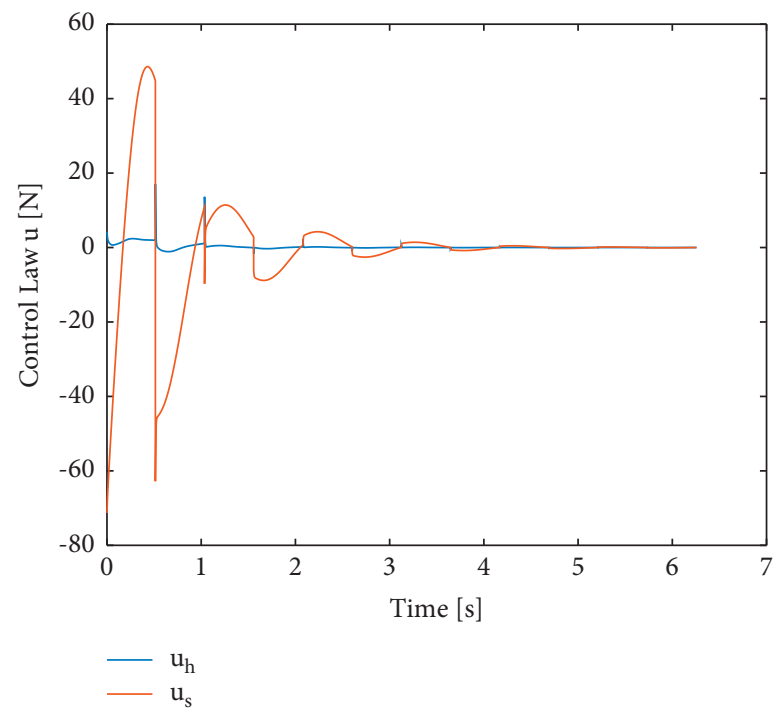

(c)

FigURE 28: Simulation results of the controlled chaotic passive gait of the bipedal walker for the set of parameters $a=0.8$ and $\varphi=8.3^{\circ}$, using the first control approach based on the passive dynamic walking.

effort of the stance leg $u_{s}$ reaches the highest value about $55[N]$. While the biped robot walks down the sloped surface, this value decreases and converges to zero. However, the control effort applied to the hip takes very small values, around $\pm 5[N]$, and this value (in module) decreases in function of steps and reaches zero. Accordingly, as in the previous scenario, the controlled gait becomes nearly passive.

By considering now the first control approach adopted in this paper, and then by selecting initial condition (69), we obtain the simulation results in Figure 28. Relying on Figure 28(b), the controlled gait needs about 8 steps in order to be stabilized at the desired one. Moreover, according to Figure $28(\mathrm{c})$, the highest amplitude of the control law $u_{s}$ is about $-80[N]$. The amplitude of the control law $u_{s}$ and that of $u_{h}$ decrease in time and converge to zero. Hence, the controlled gait becomes, as in the OGY-based control method, passive. As noted in the previous scenario, the convergence of the control process using the first control approach based on the passive dynamic walking is faster than that using the OGY-based control method.

Figure 29 shows the simulation results using the second control approach based on tracking the 4th-order Spline trajectory. As in the previous cases, the controlled biped robot needs only one step to follow the planned trajectory. Nevertheless, as seen in Figure 29(b), this control method requires a considerable energy compared to the two other control techniques.

In order to further investigate the efficiency of the proposed control methods, we have modified the previous initial condition $x_{0}^{+}$given by (69) and we have selected the initial condition used for the simulation results in the previous section using the two proposed tracking-based 


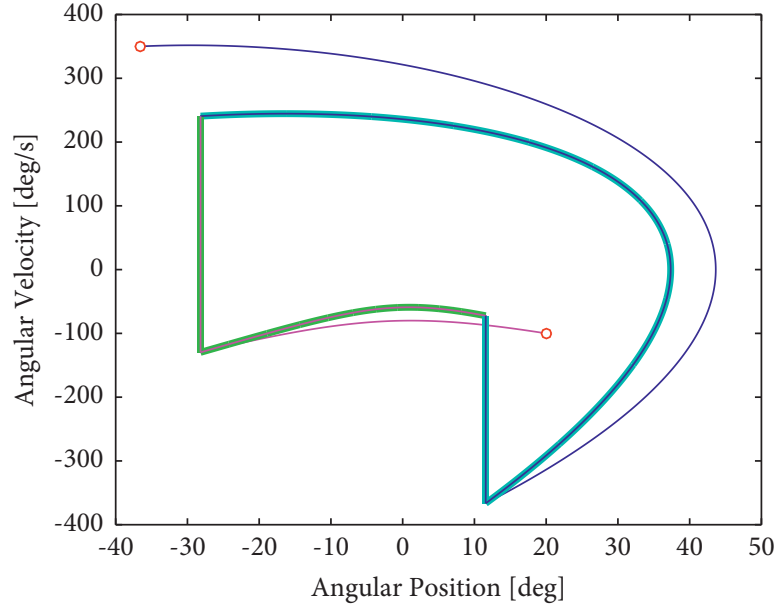

(a)

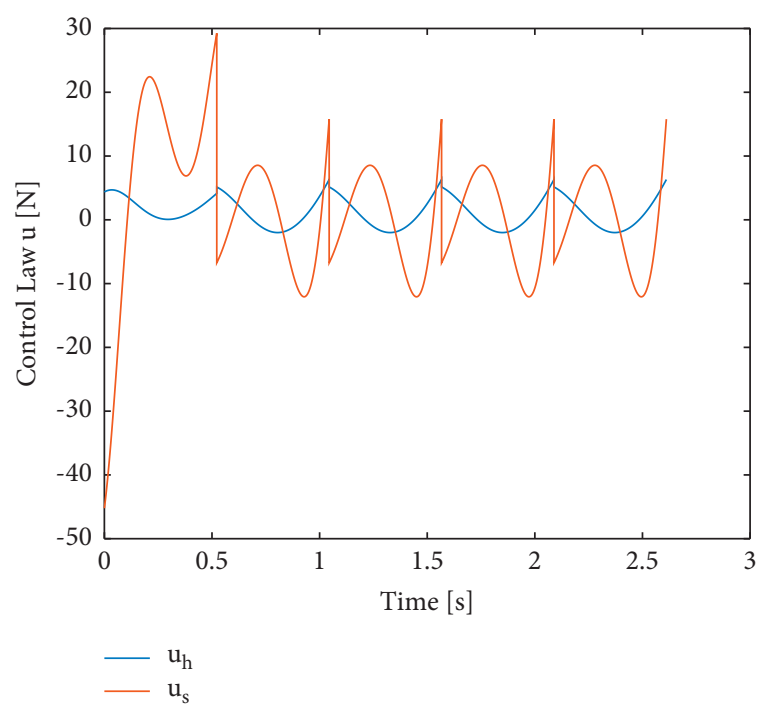

(b)

FIGURE 29: Simulation results of the controlled chaotic passive gait of the compass robot for the parameters $a=0.8$ and $\varphi=8.3^{\circ}$ by adopting the control approach based on tracking the planned 4th-order Spline trajectory.

control approaches. Such new initial condition is as follows:

$$
x_{0}^{+}=\left[\begin{array}{llll}
-21.6 & 5 & 50 & -10
\end{array}\right]
$$

Using then this initial condition $x_{0}^{+}$for the OGY-based control method, and by applying the control law (60), we found that the compass-gait bipedal robot falls down from the first step, without finishing it. As in the previous scenario, this result shows the superiority and efficiency of the two proposed control approaches compared with the OGYbased control method.

\section{Conclusion}

In this work, we have analyzed and controlled the passive dynamic walking of the compass-gait walker. For the analysis part, we have mainly used the bifurcation diagrams and we showed the production of the period-doubling route to chaos by varying either the slope angle or the length of the lower leg segment. For the control part, we have proposed two approaches for the reference trajectory tracking via the feedforward-plus-PD controller. The first approach lies mainly on the use of the generated period-1 passive gait as the reference trajectory. The second control approach results mainly in the planning of a 4 th-order Spline trajectory. Furthermore, we proposed the OGY-based control method for comparison purpose. We showed via simulation the efficiency of the proposed controller in the tracking of the desired period-1 trajectories using the two proposed control approaches. The superiority of these control approaches compared with the OGY-based control technique was also demonstrated. It was revealed that the control approach based on the passive dynamic walking provides a considerable energetic efficiency.

\section{Future Recommendations}

As a future work, we will develop other control approaches by considering also the problem of uncertainties and external disturbances. Furthermore, our goal is to extend the proposed control approaches to other models of biped robots. In addition, as a perspective that can improve our research, we will control the passive dynamic walk of the compass-type bipedal robot while minimizing energy by introducing a function of energy minimization. Moreover, in order to prevent the abrupt switching in the control law observed in almost all of the simulation results, our future direction is to use the static output feedback controller and using only the angular positions of the two legs as measurable states.

\section{Data Availability}

The data used to support the findings of this study are available from the corresponding author upon request.

\section{Conflicts of Interest}

The authors declare that there are no conflicts of interest regarding the publication of this paper.

\section{Acknowledgments}

The authors would like to thank the Ministry of Higher Education and Scientific Research (Ministère de l'Enseignement Supérieur et de la Recherche Scientifique (MESRS)), Tunisia, for technical and financial support (Project no. 20PEJC 06-02). 


\section{References}

[1] J. W. Grizzle, C. Chevallereau, R. W. Sinnet, and A. D. Ames, "Models, feedback control, and open problems of 3d bipedal robotic walking," Automatica, vol. 50, no. 8, pp. 1955-1988, 2014.

[2] E. R. Westervelt, J. W. Grizzle, C. Chevallereau, J.-H. Choi, and B. Morris, Feedback Control of Dynamic Bipedal Robot Locomotion, Taylor \& Francis/CRC, London, UK, 2007.

[3] A. Goswami and P. Vadakkepat, Humanoid Robotics: A Reference, Springer Netherlands, Berlin, Germany, 1st edition, 2019.

[4] S. Kajita, H. Hirukawa, K. Harada, K. Yokoi, and S. Sakka, Introduction à la commande des robots humanoïdes: De la modélisation à la génération du mouvement, Springer, Berlin, Germany, 2009.

[5] S. Hong, Y. Oh, D. Kim, and B.-J. You, "A walking pattern generation method with feedback and feedforward control for humanoid robots," in Proceedings of the 2009 IEEE/RSJ International Conference on Intelligent Robots and Systems, pp. 1078-1083, IEEE, St. Louis, MO, USA, October 2009.

[6] Z. Tang and M. J. Er, "Humanoid 3d gait generation based on inverted pendulum model," in Proceedings of the 2007 IEEE 22nd International Symposium on Intelligent Control, pp. 339-344, IEEE, Singapore, October 2007.

[7] B.-J. Bum-Joo Lee, D. Stonier, Y.-D. Yong-Duk Kim, J.-K. Jeong-Ki Yoo, and J.-H. Jong-Hwan Kim, "Modifiable walking pattern of a humanoid robot by using allowable $\mathrm{zmp}$ variation," IEEE Transactions on Robotics, vol. 24, no. 4, pp. 917-925, 2008.

[8] W. Takano, K. Yamane, and Y. Nakamura, "Capture database through symbolization, recognition and generation of motion patterns," in Proceedings 2007 IEEE International Conference on Robotics and Automation, pp. 3092-3097, IEEE, Roma, Italy, April 2007.

[9] K. Harada, K. Miura, M. Morisawa et al., "Toward human-like walking pattern generator," in Proceedings of the 2009 IEEE/ RSJ International Conference on Intelligent Robots and Systems, pp. 1071-1077, IEEE, St. Louis, MO, USA, October 2009.

[10] M. Zhao, J. Zhang, H. Dong, Y. Liu, L. Li, and X. Su, "Humanoid robot gait generation based on limit cycle stability," in Robot Soccer World Cup, pp. 403-413, Springer, Berlin, Germany, 2008.

[11] R. Katoh and M. Mori, "Control method of biped locomotion giving asymptotic stability of trajectory," Automatica, vol. 20, no. 4, pp. 405-414, 1984.

[12] Q. Huang, S. Kajita, N. Koyachi et al., "A high stability, smooth walking pattern for a biped robot,"vol. 1, pp. 65-71, in Proceedings 1999 IEEE International Conference on Robotics and Automation (Cat. No. 99CH36288C), vol. 1, pp. 65-71, IEEE, Detroit, MI, USA, May 1999.

[13] H. Gritli and S. Belghith, "Identification, stability and stabilization of limit cycles in a compass-gait biped model via a hybrid Poincaré map," Advances and Applications in Nonlinear Control Systems, Springer International Publishing, Berlin, Germany, pp. 259-289, 2016.

[14] X. Wang, A. Wang, D. Wang, W. Wang, B. Liang, and Y. Qi, "Repetitive control scheme of robotic manipulators based on improved B-spline function," Complexity, vol. 2021, p. $651105,2021$.

[15] A. Gasparetto and V. Zanotto, "A new method for smooth trajectory planning of robot manipulators," Mechanism and Machine Theory, vol. 42, no. 4, pp. 455-471, 2007.
[16] P. Tangpattanakul and P. Artrit, "Minimum-time trajectory of robot manipulator using harmony search algorithm,"vol. 1, pp. 354-357, in Proceedings of the 2009 6th International Conference on Electrical Engineering/Electronics, Computer, Telecommunications and Information Technology, vol. 1, IEEE, Chonburi, Thailand, May 2009.

[17] X. Yu, W. He, H. Li, and J. Sun, “Adaptive fuzzy full-state and output-feedback control for uncertain robots with output constraint," IEEE Transactions on Systems, Man, and Cybernetics, Systems.

[18] W. He, H. Gao, C. Zhou, C. Yang, and Z. Li, "Reinforcement learning control of a flexible two-link manipulator: an experimental investigation," IEEE Transactions on Systems, Man, and Cybernetics.

[19] S. H. Strogatz, "Nonlinear Dynamics and Chaos: With Applications to Physics, Biology, Chemistry, and Engineering," Studies in Nonlinearity, Westview Press, Colorado, UK, second edition, 2014.

[20] O. Makarenkov and J. S. Lamb, "Dynamics and bifurcations of nonsmooth systems: a survey," Physica D: Nonlinear Phenomena, vol. 241, no. 2, pp. 1826-1844, 2012.

[21] S. Vaidyanathan and C. Volos, "Advances and applications in chaotic systems," Studies in Computational Intelligence, Vol. 636, Springer International Publishing, Berlin, Germany, 1st edition, 2016.

[22] A. T. Azar and S. Vaidyanathan, "Chaos modeling and control systems design," Studies in Computational Intelligence, Vol. 581, Springer International Publishing, Berlin, Germany, 1st edition, 2015.

[23] A. Buscarino, C. Famoso, L. Fortuna, and M. Frasca, "A new chaotic electro-mechanical oscillator," International Journal of Bifurcation and Chaos, vol. 26, no. 10, p. 1650161, 2016.

[24] H. Gritli and S. Belghith, "Diversity in the nonlinear dynamic behavior of a one-degree-of-freedom impact mechanical oscillator under OGY-based state-feedback control law: order, chaos and exhibition of the border-collision bifurcation," Mechanism and Machine Theory, vol. 124, pp. 1-41, 2018.

[25] C. K. Volos, I. M. Kyprianidis, and I. N. Stouboulos, "Experimental investigation on coverage performance of a chaotic autonomous mobile robot," Robotics and Autonomous Systems, vol. 61, no. 12, pp. 1314-1322, 2013.

[26] S. Iqbal, X. Zang, Y. Zhu, and J. Zhao, "Bifurcations and chaos in passive dynamic walking: a review," Robotics and Autonomous Systems, vol. 62, no. 6, pp. 889-909, 2014.

[27] A. Goswami, B. Thuilot, and B. Espiau, "A study of the passive gait of a compass-like biped robot," The International Journal of Robotics Research, vol. 17, no. 12, pp. 1282-1301, 1998.

[28] A. Goswami, B. Thuilot, and B. Espiau, "Compass-like biped robot. Part I: Stability and bifurcation of passive gaits," Technical Report 2996, INRIA, Rocquencourt, France, 1996.

[29] M. Fathizadeh, S. Taghvaei, and H. Mohammadi, "Analyzing bifurcation, stability and chaos for a passive walking biped model with a sole foot," International Journal of Bifurcation and Chaos, vol. 28, no. 9, p. 1850113, 2018.

[30] S. Montazeri Moghadam, M. Sadeghi Talarposhti, A. Niaty, F. Towhidkhah, and S. Jafari, "The simple chaotic model of passive dynamic walking," Nonlinear Dynamics, vol. 93, no. 3, pp. 1183-1199, 2018.

[31] M. Nourian Zavareh, F. Nazarimehr, K. Rajagopal, and S. Jafari, "Hidden attractor in a passive motion model of compass-gait robot," International Journal of Bifurcation and Chaos, vol. 28, no. 14, p. 1850171, 2018. 
[32] J. He and G. Ren, "On the stability of passive dynamic walker with flat foot and series ankle spring," Advances in Mechanical Engineering, vol. 10, no. 3, pp. 1-12, 2018.

[33] M. Fathizadeh, H. Mohammadi, and S. Taghvaei, "A modified passive walking biped model with two feasible switching patterns of motion to resemble multi-pattern human walking," Chaos, Solitons \& Fractals, vol. 127, pp. 83-95, 2019.

[34] Y. Kuang and Y. Shen, "Painlevé paradox and dynamic selflocking during passive walking of bipedal robot," European Journal of Mechanics - A: Solids, vol. 77, p. 103811, 2019.

[35] K. Okamoto, S. Aoi, I. Obayashi, H. Kokubu, K. Senda, and K. Tsuchiya, "Fractal mechanism of basin of attraction in passive dynamic walking," Bioinspiration \& Biomimetics, vol. 15, no. 5, Article ID 055002, 2020.

[36] H. Gritli, S. Belghith, and N. Khraief, "Cyclic-fold bifurcation and boundary crisis in dynamic walking of biped robots," International Journal of Bifurcation and Chaos, vol. 22, no. 10, p. 1250257, 2012.

[37] Q. Li, J. Guo, and X.-S. Yang, "Bifurcation and chaos in the simple passive dynamic walking model with upper body," Chaos: An Interdisciplinary Journal of Nonlinear Science, vol. 24, no. 3, Article ID 033114, 2014.

[38] H. Gritli, N. Khraief, and S. Belghith, "Period-three route to chaos induced by a cyclic-fold bifurcation in passive dynamic walking of a compass-gait biped robot," Communications in Nonlinear Science and Numerical Simulation, vol. 17, no. 11, pp. 4356-4372, 2012.

[39] Q. Li, S. Tang, and X.-S. Yang, "New bifurcations in the simplest passive walking model," Chaos: An Interdisciplinary Journal of Nonlinear Science, vol. 23, no. 4, Article ID 043110, 2013.

[40] H. Gritli and S. Belghith, "Walking dynamics of the passive compass-gait model under OGY-based control: emergence of bifurcations and chaos," Communications in Nonlinear Science and Numerical Simulation, vol. 47, pp. 308-327, 2017.

[41] H. Gritli and S. Belghith, "Walking dynamics of the passive compass-gait model under OGY-based state-feedback control: analysis of local bifurcations via the hybrid Poincaré map," Chaos, Solitons \& Fractals, vol. 98, pp. 72-87, 2017.

[42] H. Gritli and S. Belghith, "Walking dynamics of the passive compass-gait model under OGY-based state-feedback control: rise of the Neimark-Sacker bifurcation," Chaos, Solitons \& Fractals, vol. 110, pp. 158-168, 2018.

[43] A. L. Fradkov and R. J. Evans, "Control of chaos: methods and applications in engineering," Annual Reviews in Control, vol. 29, no. 1, pp. 33-56, 2005.

[44] A. L. Fradkov, R. J. Evans, and B. R. Andrievsky, "Control of chaos: methods and applications in mechanics," Philosophical Transactions of The Royal Society A, vol. 364, pp. 2279-2307, 1846.

[45] B. R. Andrievskii and A. L. Fradkov, "Control of chaos: methods and applications. I. Methods," Automation and Remote Control, vol. 64, no. 5, pp. 673-713, 2003.

[46] B. R. Andrievskii and A. L. Fradkov, "Control of chaos: methods and applications. II. Applications," Automation and Remote Control, vol. 65, no. 4, pp. 505-533, 2004.

[47] S. Boccaletti, C. Grebogi, Y.-C. Lai, H. Mancini, and D. Maza, "The control of chaos: theory and applications," Physics Reports, vol. 329, no. 3, pp. 103-197, 2000.

[48] H. Karami, S. Mobayen, M. Lashkari, F. Bayat, and A. Chang, "Lmi-observer-based stabilizer for chaotic systems in the existence of a nonlinear function and perturbation," Mathematics, vol. 9, no. 10, p. 1128, 2021.
[49] S. M. Esmaeilzadeh, M. Golestani, and S. Mobayen, "Chattering-free fault-tolerant attitude control with fast fixed-time convergence for flexible spacecraft," International Journal of Control, Automation and Systems, vol. 19, no. 2, pp. 767-776, 2021.

[50] G. Pujol-Vazquez, S. Mobayen, and L. Acho, "Robust control design to the furuta system under time delay measurement feedback and exogenous-based perturbation," Mathematics, vol. 8 , no. 12, p. 2131, 2020.

[51] S. Rasoolinasab, S. Mobayen, A. Fekih, P. Narayan, and Y. Yao, "A composite feedback approach to stabilize nonholonomic systems with time varying time delays and nonlinear disturbances," ISA Transactions, vol. 101, pp. 177-188, 2020.

[52] S. Gupta and A. Kumar, "A brief review of dynamics and control of underactuated biped robots," Advanced Robotics, vol. 31, no. 12, pp. 607-623, 2017.

[53] K. Deng, M. Zhao, and W. Xu, "Level-ground walking for a bipedal robot with a torso via hip series elastic actuators and its gait bifurcation control," Robotics and Autonomous Systems, vol. 79, pp. 58-71, 2016.

[54] K. Deng, M. Zhao, and W. Xu, "Bifurcation gait suppression of a bipedal walking robot with a torso based on model predictive control," Robotics and Autonomous Systems, vol. 89, pp. 27-39, 2017.

[55] Y. Huang, Q. Huang, and Q. Wang, "Chaos and bifurcation control of torque-stiffness-controlled dynamic bipedal walking," IEEE Transactions on Systems, Man, and Cybernetics: Systems, vol. 47, no. 7, pp. 1229-1240, 2017.

[56] S. Taghvaei and R. Vatankhah, "Detection of unstable periodic orbits and chaos control in a passive biped model," Iranian Journal of Science and Technology, Transactions of Mechanical Engineering, vol. 40, no. 4, pp. 303-313, 2016.

[57] V. de León-Gómez, V. Santibañez, and J. Sandoval, "Interconnection and damping assignment passivity-based control for a compass-like biped robot," International Journal of Advanced Robotic Systems, vol. 14, no. 4, p. $1729881417716593,2017$.

[58] S. Collins, A. Ruina, R. Tedrake, and M. Wisse, "Efficient bipedal robots based on passive-dynamic walkers," Science, vol. 307, no. 5712, pp. 1082-1085, 2005.

[59] D. Koop and C. Q. Wu, "Passive dynamic biped walking-Part I: development and validation of an advanced model," Journal of Computational and Nonlinear Dynamics, vol. 8, Article ID 041007, 2013.

[60] D. Koop and C. Q. Wu, "Passive dynamic biped walking-Part II: stability analysis of the passive dynamic gait," Journal of Computational and Nonlinear Dynamics, vol. 8, Article ID 041008, 2013.

[61] D. Renjewski, A. Spröwitz, A. Peekema, M. Jones, and J. Hurst, "Exciting engineered passive dynamics in a bipedal robot," IEEE Transactions on Robotics, vol. 31, no. 5, pp. 1244-1251, 2015.

[62] T. McGeer, "Passive dynamic walking," The International Journal of Robotics Research, vol. 9, no. 2, pp. 62-82, 1990.

[63] M. Wisse, G. Feliksdal, J. V. Frankenhuyzen, and B. Moyer, "Passive-based walking robot," IEEE Robotics and Automation Magazine, vol. 14, no. 2, pp. 52-62, 2007.

[64] M. Garcia, A. Chatterjee, and A. Ruina, "Efficiency, speed, and scaling of two-dimensional passive-dynamic walking," $D y$ namics and Stability of Systems, vol. 15, no. 2, pp. 75-99, 2000.

[65] F. Asano, Z. W. Zhi-Wei Luo, and M. Yamakita, "Biped gait generation and control based on a unified property of passive 
dynamic walking," IEEE Transactions on Robotics, vol. 21, no. 4, pp. 754-762, 2005.

[66] L. Ning, L. Junfeng, and W. Tianshu, "The effects of parameter variation on the gaits of passive walking models: simulations and experiments," Robotica, vol. 27, no. 4, pp. 511-528, 2009.

[67] K. Deng, M. Zhao, and W. Xu, "Passive dynamic walking with a torso coupled via torsional springs," International Journal of Humanoid Robotics, vol. 13, no. 4, p. 1650024, 2017.

[68] H. Razavi, A. M. Bloch, C. Chevallereau, and J. W. Grizzle, "Symmetry in legged locomotion: a new method for designing stable periodic gaits," Autonomous Robots, vol. 41, no. 5, pp. 1119-1142, 2017.

[69] F. Iida and R. Tedrake, "Minimalistic control of biped walking in rough terrain," Autonomous Robots, vol. 28, no. 3, pp. 355-368, 2010.

[70] K. Byl and R. Tedrake, "Metastable walking machines," The International Journal of Robotics Research, vol. 28, no. 8, pp. 1040-1064, 2009.

[71] H. Gritli, N. Khraief, and S. Belghith, "Chaos control in passive walking dynamics of a compass-gait model," Communications in Nonlinear Science and Numerical Simulation, vol. 18, no. 8, pp. 2048-2065, 2013.

[72] W. Znegui, H. Gritli, and S. Belghith, "Stabilization of the passive walking dynamics of the compass-gait biped robot by developing the analytical expression of the controlled Poincaré map," Nonlinear Dynamics, vol. 101, no. 2, pp. 1061-1091, 2020.

[73] H. Sadeghian and M. Barkhordari, "Orbital analysis of passive dynamic bipeds; the effect of model parameters and stabilizing arm," International Journal of Mechanical Sciences, vol. 178, p. 105616, 2020.

[74] O. Makarenkov, "Existence and stability of limit cycles in the model of a planar passive biped walking down a slope," Proceedings of the Royal Society A: Mathematical, Physical \& Engineering Sciences, vol. 476, no. 2233, p. 20190450, 2020.

[75] J. N. Martínez-Castelán and M. G. Villarreal-Cervantes, "Frontal-sagittal dynamic coupling in the optimal design of a passive bipedal walker," IEEE Access, vol. 7, pp. 427-449, 2019.

[76] A. T. Safa, S. Mohammadi, S. E. Hajmiri, M. Naraghi, and A. Alasty, "How local slopes stabilize passive bipedal locomotion?" Mechanism and Machine Theory, vol. 100, pp. 6382, 2016.

[77] E. Added and H. Gritli, "Control of the passive dynamic gait of the bipedal compass-type robot through trajectory tracking," in Proceedings of the 2020 20th International Conference on Sciences and Techniques of Automatic Control and Computer Engineering (STA), pp. 155-162, Monastir, Tunisia, December 2020.

[78] E. Added and H. Gritli, "Trajectory design and tracking-based control of the passive compass biped," in Proceedings of the 2020 4th International Conference on Advanced Systems and Emergent Technologies (IC_ASET), pp. 417-424, Hammamet, Tunisia, March 2020.

[79] I. A. Hiskens and M. A. Pai, "Trajectory sensitivity analysis of hybrid systems," IEEE Transactions on Circuits and Systems I: Fundamental Theory and Applications, vol. 47, no. 2, pp. 204-220, 2000.

[80] H. Yuan, Y. Ge, and C. Gan, "Modification-based periodic gait planning for a 3-D bipedal robot with two underactuated DOFs," Asian Journal of Control, pp. 1-13, 2020.

[81] H. Wang, H. Zhang, Z. Wang, and Q. Chen, "Finite-time stabilization of periodic orbits for under-actuated biped walking with hybrid zero dynamics," Communications in
Nonlinear Science and Numerical Simulation, vol. 80, p. 104949, 2020.

[82] H. Gritli, S. Belghith, and N. Khraief, "OGY-based control of chaos in semi-passive dynamic walking of a torso-driven biped robot," Nonlinear Dynamics, vol. 79, no. 2, pp. 1363-1384, 2015.

[83] H. Gritli and S. Belghith, "Displayed phenomena in the semipassive torso-driven biped model under OGY-based control method: birth of a torus bifurcation," Applied Mathematical Modelling, vol. 40, no. 4, pp. 2946-2967, 2016.

[84] W. Znegui, H. Gritli, and S. Belghith, "Design of an explicit expression of the Poincaré map for the passive dynamic walking of the compass-gait biped model," Chaos, Solitons \& Fractals, vol. 130, p. 109436, 2020.

[85] W. Znegui, H. Gritli, and S. Belghith, "A new Poincaré map for investigating the complex walking behavior of the compass-gait biped robot," Applied Mathematical Modelling, vol. 94, pp. 534-557, 2021. 